\title{
$\checkmark$ CIRANO
}

Allier savoir et décision

\section{INNOVATION OUVERTE ET ÉCOSYSTÈME D'INNOVATION : IMPLICATIONS POUR LES ORGANISATIONS DU SECTEUR PUBLIC}

SIMON BOURDEAU THIBAUT COULON AMANDINE PASCAL MATHIEU TEMPLIER 
Les rapports de projet sont destinés plus spécifiquement aux partenaires et à un public informé. Ils ne sont ni écrits à des fins de publication dans des revues scientifiques ni destinés à un public spécialisé, mais constituent un médium d'échange entre le monde de la recherche et le monde de la pratique.

Project Reports are specifically targeted to our partners and an informed readership. They are not destined for publication in academic journals nor aimed at a specialized readership, but are rather conceived as a medium of exchange between the research and practice worlds.

Le CIRANO est un organisme sans but lucratif constitué en vertu de la Loi des compagnies du Québec. Le financement de son infrastructure et de ses activités de recherche provient des cotisations de ses organisations-membres, d'une subvention d'infrastructure du gouvernement du Québec, de même que des subventions et mandats obtenus par ses équipes de recherche.

CIRANO is a private non-profit organization incorporated under the Quebec Companies Act. Its infrastructure and research activities are funded through fees paid by member organizations, an infrastructure grant from the government of Quebec, and grants and research mandates obtained by its research teams.

Les partenaires du CIRANO - CIRANO Partners

Partenaires corporatifs - Corporate Partners

Autorité des marchés financiers

Banque de développement du Canada

Banque du Canada

Banque nationale du Canada

Bell Canada

BMO Groupe financier

Caisse de dépôt et placement du Québec

Énergir

Hydro-Québec

Innovation, Sciences et Développement économique Canada

Intact Corporation Financière

Investissements PSP

Manuvie Canada

Ministère de l'Économie, de la Science et de l'Innovation

Ministère des finances du Québec

Mouvement Desjardins

Power Corporation du Canada

Rio Tinto

Ville de Montréal

Partenaires universitaires - Academic Partners

École de technologie supérieure

École nationale d'administration publique

HEC Montréal

Institut national de la recherche scientifique

Polytechnique Montréal

Université Concordia

Université de Montréal

Université de Sherbrooke

Université du Québec

Université du Québec à Montréal

Université Laval

Université McGill

Le CIRANO collabore avec de nombreux centres et chaires de recherche universitaires dont on peut consulter la liste sur son site web. CIRANO collaborates with many centers and university research chairs; list available on its website.

(c) Novembre 2021. Simon Bourdeau, Thibaut Coulon, Amandine Pascal et Mathieu Templier. Tous droits réservés. All rights reserved. Reproduction partielle permise avec citation du document source, incluant la notice $@$. Short sections may be quoted without explicit permission, if full credit, including (C) notice, is given to the source.

Les idées et les opinions émises dans cette publication sont sous l'unique responsabilité des auteurs et ne représentent pas nécessairement les positions du CIRANO ou de ses partenaires. The observations and viewpoints expressed in this publication are the sole responsibility of the authors; they do not necessarily represent the positions of CIRANO or its partners.

ISSN 1499-8629 (version en ligne) 


\title{
Innovation ouverte et écosystème d'innovation : Implications pour les organisations du secteur public
}

\author{
Simon Bourdeau*, Thibaut Coulon ${ }^{\dagger}$, Amandine Pascal ${ }^{\ddagger}$ et Mathieu Templier ${ }^{\S}$
}

Novembre 2021

\begin{abstract}
Résumé
Le déploiement d'initiatives d'innovation ouverte représente, pour tous types d'organisations et plus particulièrement pour les organisations du secteur public (OSP), une aventure risquée et exigeante. La présente étude a identifié 17 défis, regroupés dans quatre catégories, liés au déploiement d'IO : 1) Collaboration inter-organisationnelle, 2) Processus d'innovation, 3) Implication des citoyens et 4) Données et technologies, ainsi que 18 bonnes pratiques/actions organisationnelles permettant d'augmenter les probabilités de succès. Finalement, deux études de cas, PULSAR ${ }^{* *}$ et Cité de l'innovation et des savoirs Aix-Marseille $\left(\right.$ CISAM) ${ }^{\dagger+}$, ont été réalisées, afin de mieux comprendre comment ces pratiques peuvent répondre aux défis, tout en en favorisant la création de valeur lors du cycle d'innovation.
\end{abstract}

Mots-clés : Innovation ouverte $(10)$, organisations du secteur public, défis 10 , bonnes pratiques IO, capacités dynamiques, études de cas

Codes JEL : O36, O38, O30

\begin{abstract}
Deploying open innovation initiatives is a risky and demanding venture for all types of organizations, but especially for public sector organizations (PSOs). This study identified 17 challenges, grouped in four categories, related to Ol deployment: 1) Inter-organizational collaboration, 2) Innovation process, 3) Citizen engagement, and 4) Data and technology, as well as 18 good practices/organizational actions to increase the probability of success. Finally, two case studies, PULSAR and Cité de l'innovation et des savoirs Aix-Marseille (CISAM), were conducted to better understand how these practices can meet the challenges, while promoting value creation during the innovation cycle.
\end{abstract}

Keywords : Open Innovation (OI), public sector organizations, OI challenges, OI best practices, dynamic capabilities, case studies

\footnotetext{
*Professeur agrégé, ESG-UQAM et Fellow CIRANO, bourdeau.s@uqam.ca

† Professeur adjoint, ESG-UQAM, coulon.thibaut@uqam.ca

₹ Professeur agrégée, Aix-Marseille Université, amandine.pascal@univ-amu.fr

§ Professeur agrégé, Université Laval, mathieu.templier@fsa.ulaval.ca

${ }^{* *}$ https://pulsar.ca/accueil

t† https://cisam-innovation.com/
} 


\section{Pour citer ce document / To quote this document}

Bourdeau, S., Coulon, T., Pascal, A. and Templier, M. (2021). Innovation ouverte et écosystème d'innovation : Implications pour les organisations du secteur public (2022s05). CIRANO. https://doi.org/10.54932/NIBF5951 


\section{Sommaire exécutif}

Ce projet de recherche focalise sur de nouvelles approches d'ouverture et de collaboration dans le cycle d'innovation d'organisations du secteur public (OSP). Les objectifs du projet sont de :

(1) Recenser et analyser les défis et les bonnes pratiques mises en place lors d'une démarche d'innovation ouverte (IO), impliquant des organisations publiques, dans un contexte d'écosystème d'innovation,

(2) Comprendre, à travers deux études de cas d'écosystème d'innovation, comment ces pratiques peuvent répondre aux principaux défis rencontrés pour favoriser la création de valeur lors du cycle d'innovation.

Au total, ce sont 17 défis reliés au déploiement d'IO par des OSP, et regroupé dans quatre catégories, qui ont été identifiés. Le tableau S1 présente une synthèse de ces défis qui sont décrits en détails dans la section 3 du rapport.

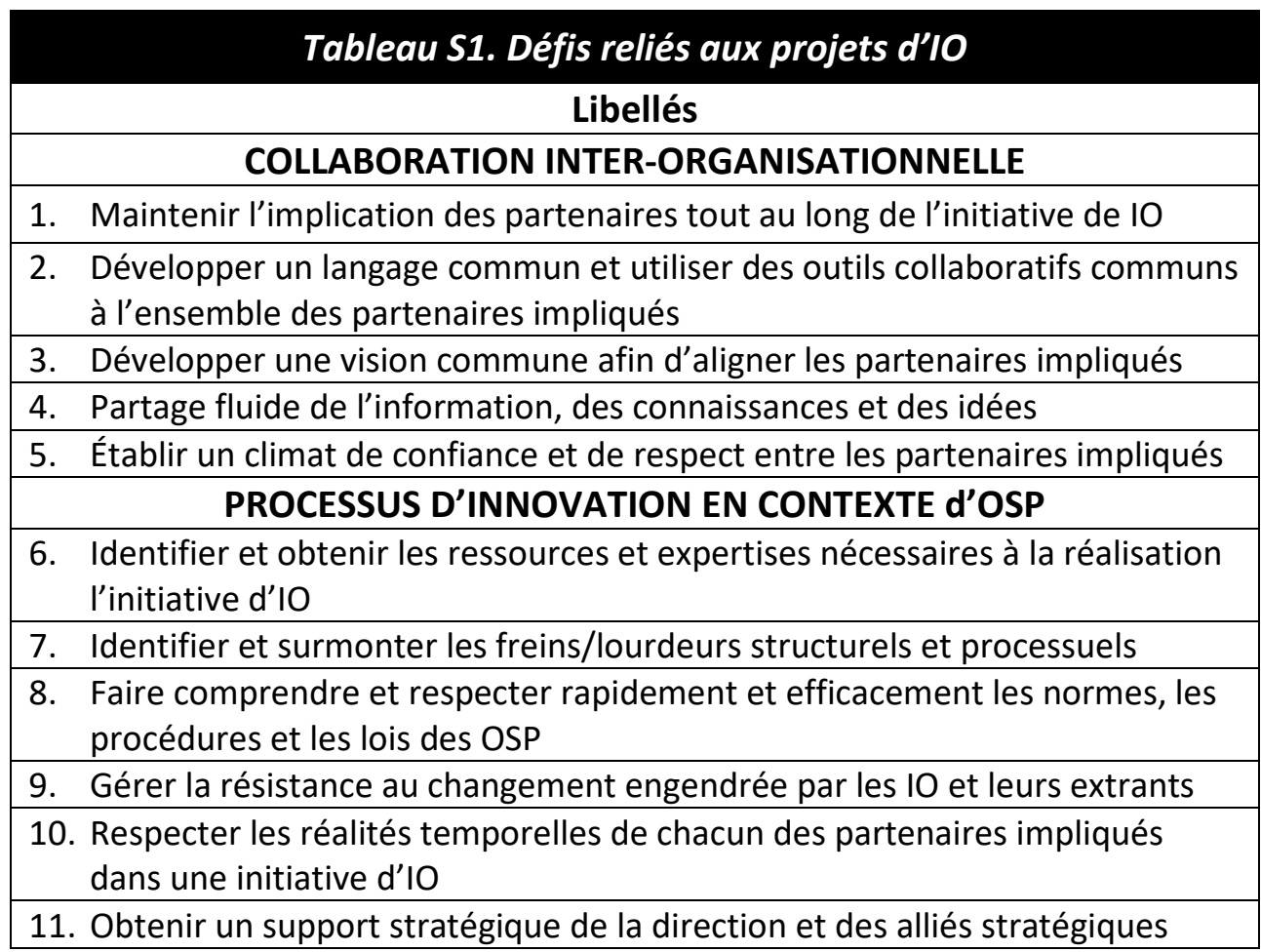


12. Prendre en compte et anticiper les enjeux politiques afin d'apporter les ajustements nécessaires

\section{IMPLICATION DES CITOYENS}

13. Bien comprendre et connaître les particularités et le contexte des citoyens afin d'accroître l'inclusion sociale

14. Identifier et utiliser les canaux de communication adaptés aux citoyens

15. Obtenir et maintenir l'engagement des citoyens participants

\section{DONNÉES ET TECHNOLOGIES}

16. Surmonter les limites des infrastructures technologiques existantes

17. Assurer une gouvernance sécuritaire des données (acquisition, stockage, partage)

$\mathrm{Au}$ total, 18 bonnes pratiques/actions organisationnelles déployées par les OSP pour supporter la mise en œuvre d'initiatives d'IO en contexte d'écosystème d'innovation ont été identifiées. Le tableau S2 présente une synthèse de ces actions organisationnelles qui sont décrites en détails dans la section 3 du rapport.

\section{Tableau 2. Actions organisationnelles déployées par les OSP lors} d'Initiatives d'IO

1. Favoriser une culture d'ouverture et d'expérimentation

2. Impliquer les partenaires à l'aide de plateformes collaboratives et coordonner les échanges

3. Générer et collecter des idées à l'aide d'une plateforme numérique

4. Développer une présence sur les réseaux sociaux

5. Organiser des rencontres entre les partenaires clés impliqués dans l'IO

6. Cartographier les besoins et les capacités des utilisateurs finaux

7. Développer des prototypes et des prétests fréquemment

8. Adopter une approche agile

9. Gérer les enjeux relatifs à la gouvernance et à la sécurité des données

10. Promouvoir une communication ouverte et transparente entre les partenaires

11. Renforcer la motivation et l'engagement des partenaires

12. Allouer des ressources financières suffisantes aux initiatives d'IO

13. Obtenir un support des hauts dirigeants

14. Développer un leadership fort et une vision claire et cohérente

15. Identifier et sélectionner des membres d'équipe avec des compétences complémentaires et une bonne attitude

16. Utiliser un cadre de travail collaboratif commun

17. Surveiller le déroulement des initiatives d'IO

18. Déployer une infrastructure informatique flexible et des données ouvertes 
Finalement, afin de mieux comprendre comment les bonnes pratiques identifiées peuvent aider les OSP à répondre aux défis liés à I'IO, tout en en favorisant la création de valeur lors du cycle d'innovation, deux études de mise en place d'écosystèmes d'innovation, impliquant des OSP, ont été réalisées. Tout d'abord, une première étude de cas a été réalisée au Québec chez PULSAR ${ }^{5}$ qui est un espace collaboratif de recherche et d'innovation en santé durable mise en œuvre par l'Université Laval et Alliance Santé Québec (Canada). Une seconde étude de cas a été réalisée en France avec la Cité de l'innovation et des savoirs Aix-Marseille (CISAM) ${ }^{6}$, qui est un espace unique de créativité entrepreneuriale, de rencontres et d'enrichissement sur le territoire d'Aix-Marseille (France). La section 4 présente la description et l'analyse de ces deux cas d'écosystèmes d'IO et illustrent comment chacun des 18 actions organisationnelles (voir tableaux 3 et 4 ) peuvent être concrètement mise en place.

${ }^{5}$ https://pulsar.ca/accueil

${ }^{6}$ https://cisam-innovation.com/ 


\section{Table des matières}

$1 \quad$ Mise en contexte, rappel du mandat et structure du document

1.1 L'Innovation dans le secteur public 7

1.2 Objectif et méthodologie de recherche 11

2 Quelques définitions et fondements théoriques 15

2.1 Innovation, capacités dynamiques et écosystème 15

2.2 Innovation et innovation ouverte dans le secteur public _ 19

2.3 Capacité dynamique, innovation ouverte et écosystème_ 21

3 Recension de cas " exemplaires " d'innovations ouvertes dans un contexte d'écosystème d'innovation

3.1 Défis reliés au déploiement d'initiatives d'IO menées par des OSP

3.2 Bonnes pratiques/actions organisationnelles déployées par les OSP lors d'initiatives d'IO 33

3.3 Analyse des bonnes pratiques liées à la DÉTECTION Détecter les opportunités ou les menaces 47

3.4 Analyse des bonnes pratiques liées à la CAPTURE - Saisir les opportunités et éviter les menaces 49

3.5 Analyse des bonnes pratiques liées à la TRANSFORMATION - Mobiliser, combiner et reconfigurer les actifs/capacités 51

3.6 Bonnes pratiques d'innovation ouverte et rôle des technologies de l'information 54

4 Écosystème d'innovation : Deux études de cas :

PULSAR et CISAM 56

4.1 Étude de cas : PULSAR et CISAM 56

4.1.1 PULSAR - Description, partenaires et santé durable 56

4.1.2 PULSAR - Mission, cadre d'évaluation de la santé durable et services technologiques 59

4.1.3 PULSAR - Pratiques déployées pour favoriser l'innovation ouverte 63

4.1.4 CISAM - Description, structure et objectifs 75

4.1.5 CISAM - Pratiques déployées pour favoriser l'innovation ouverte 81 
Annexe 1 - Explications théoriques complémentaires

Processus d'innovation et l'innovation ouverte

Écosystème : Comme une affiliation ou comme une structure 92

Écosystème vs Réseau vs Marché 94

Genèse d'écosystèmes et rôles clés 96

Annexe 2-Recensement défis et bonnes pratiques 99

Annexe 3-Études de cas : PULSAR et CISAM 103

Annexe 4-Guide d'entrevue 105

Références bibliographiques 108 


\section{Innovation ouverte et écosystème d'innovation : Implications pour le secteur public}




\section{Mise en contexte, rappel du mandat et structure du document}

\subsection{L'Innovation dans le secteur public}

\section{Pourquoi innover : Les défis dans le secteur public}

Les organisations du secteur public (OSP) font face à des défis sans précédent engendrés par des crises financières, des pandémies, la baisse des naissances et le vieillissement de la population, les changements climatiques et environnementaux, la diminution des ressources, la "consumérisation" des services publics ou encore la constante et rapide évolution des technologies ${ }^{7}$. Tous ces facteurs interdépendants créent un environnement volatil, incertain et de plus en plus complexe dans lequel les OSP doivent naviguer. Pour s'adapter et survivre dans un tel

L'innovation est une réponse aux multiples défis actuels auxquels sont confrontées aussi bien les organisations privées que publiques. environnement, les OSP doivent se réinventer, elles doivent innover.

L'innovation est une réponse aux multiples défis actuels auxquels sont confrontées aussi bien les organisations privées que publiques. L'innovation représente l'application d'une nouvelle idée qui induit un changement dans la pratique avec un objectif de création de valeur. Le processus d'innovation consiste alors à vouloir améliorer l'existant de façon significative en transformant des idées novatrices en solutions concrètes qui seront implantées dans l'organisation. II s'agit d'un processus dynamique par lequel des agents, des organisations, des institutions et des structures de l'économie sont transformés par les effets d'idées novatrices ${ }^{8}$. Le processus " classique " d'innovation se divise en cinq étapes: 1) Formulation du problème, 2) Génération d'idées pour résoudre le problème, 3) Sélection d'idées, 4) Conversion d'idées en solutions concrètes et, finalement, 5) Diffusion de I'innovation ${ }^{9}$. Afin de favoriser l'innovation dans les

\footnotetext{
5 (Fernandez and Rainey 2006; Tat-Kei Ho 2002; Wiesel and Modell 2014)

8 (Hansen and Birkinshaw 2007; Potts and Kastelle 2010)

9 (Eggers and Singh 2009; Rogers 2003)
} 
Pour répondre à

l'ensemble de ces

pressions et s'adapter

aux mouvements de

réformes, les OSP

doivent donc se

réinventer et innover. organisations, mais aussi dans la société, il est essentiel que les OSP comprennent comment promouvoir, supporter et gérer chacune des étapes du processus d'innovation. II a été démontré que l'innovation peut, dans le secteur public, contribuer à ${ }^{10}$ :

1) Améliorer la qualité, l'efficacité et l'efficience des services publics offerts,

2) Augmenter la satisfaction des citoyens,

3) Améliorer la capacité de résolution des problèmes complexes des OSP,

4) Acquérir la confiance et la légitimité,

5) Attirer des ressources humaines de haut niveau,

6) Favoriser le capital social et l'activisme local, et

7) Promouvoir la participation de citoyens et de partenaires privés.

Il existe de nombreux facteurs qui incitent les OSP à innover. D'un côté, tant les citoyens et les communautés que les organismes et entreprises privées ont des attentes de plus en grandes en termes de qualité, de disponibilité, de flexibilité, de personnalisation et d'efficacité des services offerts par les OSP ${ }^{11}$. De l'autre côté, les OSP subissent des pressions structurelles et conjecturales qui limitent leurs ressources, ainsi que des pressions environnementales qui restreignent leurs champs d'action (p. ex. scrutation des décisions et actions par les médias, accroissement des demandes provenant d'une multitude de partenaires impliqués, devoir d'être un citoyen exemplaire en terme social, légal, environnemental, économique, etc.). Cette situation force ainsi les OSP à identifier et développer de nouveaux modes de prestation de services publics ${ }^{12}$.

De plus, les OSP subissent également des pressions des grands mouvements de réforme du secteur public tel que le

\footnotetext{
10 (Bekkers et al. 2011; Damanpour and Schneider 2009; De Vries et al. 2015; Green et al. 2014; Koppenjan and Klijn 2004; Linders 2012)

11 (Sørensen and Torfing 2011; Vigoda-Gadot et al. 2008).

12 (De Vries et al. 2016; Hartley et al. 2013)
} 


\section{Les organisations}

publiques sont

généralement moins

innovantes qu'elles ne

pourraient idéalement

l'être, car les innovations

dans le secteur public

sont faiblement incitées

et qu'il est difficile de

mesurer la performance

de l'innovation. gouvernement électronique, le New Public Management ou encore le mouvement d'ouverture des gouvernements exigeant d'accroître la transparence, d'améliorer la participation aux processus de gouvernance et de favoriser la collaboration ${ }^{13}$. Afin de répondre à l'ensemble de ces pressions et s'adapter aux mouvements de réformes, les OSP doivent donc se réinventer et innover.

\section{Comment les OSP peuvent mieux innover : Collaboration et intégration des connaissances}

Le problème n'est donc pas tant l'absence d'innovation dans les OSP que le fait que la plupart des innovations de secteur public sont épisodiques et principalement motivées par des événements accidentels tels que des crises, une réponse à une nouvelle législation ou encore des réductions de dépenses ${ }^{14}$. Ainsi, les OSP sont généralement moins innovantes qu'elles ne pourraient idéalement l'être. En effet, les innovations dans le secteur public, plutôt averses aux risques, sont faiblement incitées d'une part. D'autre part, les OSP évoluent dans des environnements complexes, multifonctionnels, avec des règles bureaucratiques, structurelles et juridiques ainsi que des indicateurs de performance qui tendent à freiner l'innovation ${ }^{15}$.

Par ailleurs, lorsqu'il est question d'innover, les OSP sont de plus en plus confrontées à des situations problématiques volatiles, incertaines, complexes et ambiguës ${ }^{16}$ qui, pour être surmontées, nécessitent des connaissances spécialisées provenant d'un plus grand nombre de contributeurs. Une situation volatile signifie que les caractéristiques mêmes de cette situation sont inattendues, instables ou changeantes. Elles peuvent également être de durée inconnue, sans toutefois être difficile à comprendre. Les connaissances au sujet de ces

\footnotetext{
13 (Carter and Bélanger 2005; Mergel 2015; Pollitt and Bouckaert 2011)

14 (Borins 2001; Borins 2014; Sørensen and Torfing 2011)

15 (Borins 2001; Borins 2014; Potts and Kastelle 2010; Sørensen and Torfing 2011)

16 (Bennett and Lemoine 2014)
} 


\section{La mise en place de} processus d'innovation plus performants est alors favorisée par de nouvelles formes d'interactions et d'organisations basées sur l'ouverture et la collaboration entre les différents partenaires impliqués non seulement internes aux OSP, mais également avec des partenaires impliqués externes. caractéristiques peuvent être existantes ou émergentes. Une situation incertaine signifie que le niveau de certitude des causes et des effets d'une situation peut changer et que des variations peuvent encore émerger malgré les informations recueillies sur une situation donnée. Une situation complexe implique plusieurs partenaires impliqués et comporte de nombreuses variables interconnectées. De cette complexité découle une variété et un volume d'informations pouvant être exténuants à collecter, analyser et diffuser. Finalement, une situation ambigüe signifie que les liens entre les causes et les effets d'une situation problématique sont plus ou moins clairs. Une telle situation est généralement caractérisée de situation " inconnue-inconnue " ${ }^{17}$ car aucun précédent n'existe. De telles situations problématiques peuvent difficilement être résolues par les OSP en utilisant les approches d'innovation dites «traditionnelles " ${ }^{18}$. La pandémie mondiale de COVID-19 est un très bon exemple de situation problématique volatile, incertaine, complexe et ambiguë par rapport à laquelle toutes les OSP du monde ont dû réagir en adaptant leurs pratiques et surtout en innovant collectivement.

En effet, l'innovation résulte rarement des efforts déployés par une seule personne ou une seule équipe, mais nécessite plutôt la collaboration de multiples individus, équipes, communautés et partenaires qui apportent des connaissances et des perspectives différentes ${ }^{19}$. Ainsi, pour innover, le défi consiste à identifier et intégrer ces différentes connaissances et perspectives tout au long du processus d'innovation. Pour les OSP, ceci exige de collaborer, par l'entremise entre autres d'écosystèmes d'innovations, avec de multiples partenaires privés et publics tels que les politiciens, les administrateurs, les citoyens, les universités, les instituts de recherche, les organisations privées, les experts, les groupes d'utilisateurs, les organisations communautaires, etc. ${ }^{20}$.

\footnotetext{
17 En gestion de risques, on parle de situation " unkwown-unkwwon », (Kim 2017).

18 (Koppenjan and Klijn 2004; Sørensen and Torfing 2006; Sørensen and Torfing 2011)

${ }^{19}$ (Wallin and Von Krogh 2010)

20 (Sørensen and Torfing 2011)
} 
Ainsi, la mise en place de processus d'innovation plus performants est alors favorisée par de nouvelles formes $d^{\prime}$ 'interactions et d'organisations basées sur l'ouverture et la collaboration entre les différents partenaires ${ }^{21}$ non seulement internes aux OSP, mais également avec des partenaires impliqués externes. À cet égard, la mise en place d'approches d'innovation ouvertes et d'écosystèmes d'innovation dans les OSP a été proposée afin d'améliorer la rapidité et l'efficience du cycle d'innovation, de capturer l'intelligence collective et de diversifier les sources d'expertises et de connaissances ${ }^{22}$. Bien que prometteuses, ces idées restent mal comprises, peu implantées dans le secteur public et présentent plusieurs défis spécifiques.

\subsection{Objectif et méthodologie de recherche}

Ce projet de recherche focalise sur ces nouvelles approches d'ouverture et de collaboration dans le cycle d'innovation d'OSP. Les objectifs du projet sont de :

L'innovation ouverte peut être définie comme l'intégration et la diffusion volontaire de connaissances afin d'accélérer l'innovation interne ainsi que de favoriser l'utilisation des connaissances externes.
(1) Recenser et analyser les défis et les bonnes pratiques mises en place lors d'une démarche d'innovation ouverte, impliquant des organisations publiques, dans un contexte d'écosystème d'innovation ;

(2) Comprendre, à travers deux études de cas d'écosystème d'innovation, comment ces pratiques peuvent répondre aux principaux défis rencontrés pour favoriser la création de valeur lors du cycle d'innovation.

L'innovation ouverte (10) peut être définie comme l'intégration et la diffusion volontaire de connaissances afin d'accélérer l'innovation interne ainsi que de favoriser

\footnotetext{
21 (Chesbrough 2006a; Duval and Speidel 2014)

22 (Adner and Kapoor 2010; Bason 2010; Chesbrough and Bogers 2014; West et al. 2014)
} 
L'innovation ouverte et collaborative implique la participation et

l'implication de partenaires autant internes qu'externes au niveau des différentes phases du processus d'innovation.
I'utilisation des connaissances externes ${ }^{23}$. En pratique, I'IO ne se réduit pas à une approche, mais est au contraire composée par une myriade d'approches et pratiques différentes, chacune présentant des opportunités ainsi que des défis majeurs.

Dans des OSP, les projets d'IO peuvent se réaliser en interne lorsqu'ils impliquent plusieurs départements et services, ou à l'externe lorsqu'ils impliquent plusieurs organisations ou partenaires. En réponse à des pressions de performance et de compétitivité, les organisations se regroupent alors au sein d'écosystèmes d'innovation et s'engagent dans des démarches de co-création et de co-innovation. Le présent projet de recherche se concentre sur les processus d'IO impliquant la participation de partenaires aussi bien internes qu'externes aux OSP et ce, lors des différentes phases du processus $d^{\prime}$ innovation ${ }^{24}$.

\section{Deux étapes : Recensement des défis et des bonnes pratiques + Études de cas (2)}

Dans un premier temps, afin d'identifier et de recenser des défis et de bonnes pratiques d'IO mises en place par des OSP, une analyse de 253 cas d'IO a été réalisée. Cette analyse a été effectuée à partir de données secondaires provenant de la base de données de cas de l'Organisation de Coopération et de Développement Économique (OCDE) qui recense et partage de nombreux cas d'innovation https://oecdopsi.org/case type/opsi/. Plus spécifiquement, cette base de données recueille des "cas exemplaires" d'innovations impliquant plusieurs acteurs afin que les décideurs et des innovateurs à travers la planète puissent s'en inspirer, en tirer des leçons, prennent contact avec les personnes impliquées et possiblement collaborent avec elles. Ainsi, l'analyse de ces cas d'IO, qui ont tous été des succès, a permis d'identifier des défis et de bonnes pratiques liées au cycle d'IO impliquant des OSP.

\footnotetext{
23 (Chesbrough and Bogers 2014)

${ }^{24}$ (Chesbrough 2006a; Chesbrough 2006b; Piller et al. 2012; Russo-Spena and Mele 2012)
} 
PULSAR est un espace collaboratif de recherche et d'innovation en santé durable.

\section{Cité de l'innovation et des}

savoirs Aix-Marseille

(CISAM) est un espace de créativité

entrepreneuriale, de rencontres et d'enrichissement sur le territoire d'Aix-Marseille.
Une attention particulière a été portée sur le rôle joué par les technologies de l'information (TI) afin de supporter ces initiatives $d^{\prime} 1 O^{25}$.

Dans un second temps, afin de mieux comprendre comment les bonnes pratiques identifiées peuvent aider les OSP à répondre aux défis liés à I'IO, tout en en favorisant la création de valeur lors du cycle d'innovation, deux études de mise en place d'écosystèmes d'innovation, impliquant des OSP, ont été réalisées. Tout d'abord, une première étude de cas a été réalisée au Québec chez PULSAR $^{26}$ qui est un espace collaboratif de recherche et d'innovation en santé durable mise en œuvre par I'Université Laval et Alliance Santé Québec (Canada). À la fois virtuel et réel, l'espace PULSAR rassemble des acteurs, de tout horizon, dont l'objectif est d'améliorer de façon significative et durable la santé et le bien-être de la population. Une seconde étude de cas a été réalisée en France avec la Cité de l'innovation et des savoirs Aix-Marseille $(\text { CISAM })^{27}$, qui est un espace unique de créativité entrepreneuriale, de rencontres et d'enrichissement sur le territoire d'Aix-Marseille (France). La CISAM repose sur les connaissances et les ambitions partagées par la plus grande université francophone Aix-Marseille et deux multinationales : la CMA-CGM et le Groupe L'OCCITANE. L'objectif de la CISAM est de développer une culture scientifique et d'innovation au service de l'essor économique en regroupant, sur un même site, l'ensemble des ressources permettant de stimuler des démarches innovantes. La CISAM se veut un lieu de rencontres, de création, d'incubation de projets, et d'accélération.

Afin d'identifier des défis et de bonnes pratiques liées à une démarche d'IO impliquant des OSP ainsi que de mieux comprendre comment ces pratiques peuvent répondre aux principaux défis rencontrés pour favoriser la création de valeur dans un contexte d'écosystème d'innovation, l'équipe du CIRANO s'est vue confier le mandat d'effectuer une analyse de

\footnotetext{
25 (Eggers and Singh 2009; Hoon 2013; Larsson 1993; Rogers 2003)

${ }^{26}$ https://pulsar.ca/accueil

27 https://cisam-innovation.com/
} 
cas d'innovation et une étude comparative détaillée d'écosystèmes d'innovation. Le reste du présent rapport se divise comme suit : la section 2 présente le cadre théorique sous-jacent à l'étude menée; la section 3 présente les principaux défis et pratiques liées aux initiatives d'IO impliquant des OSP ; la section 4 présente la description et l'analyse de deux cas d'écosystèmes d'IO, soit Pulsar et CISAM ; et finalement la section 5 présente une discussion des résultats de l'étude ainsi qu'une conclusion. 


\section{L'innovation est un} processus micro et macro dynamique par lequel des agents, des organisations, des institutions et des structures macro de l'économie sont transformés par les effets d'une idée nouvelle.

\section{Quelques définitions et fondements théoriques $^{28}$}

Tel que mentionné précédemment, l'évolution constante et rapide des technologies numériques, le vieillissement de la population, les changements climatiques, la diminution des ressources et les crises financières sont autant de défis auxquels sont confrontées les organisations du secteur public (OSP). Ces organisations doivent non seulement faire face à des pressions structurelles et conjecturales qui limitent leurs ressources et leurs pouvoirs d'action, mais doivent en plus répondre aux attentes, en constante croissance, des citoyens, des communautés et des organisations privées. Tous ces facteurs interdépendants créent un environnement de travail volatil, incertain, complexe et ambigu nécessitant des connaissances spécialisées de plus en plus importantes et provenant d'un nombre de contributeurs de plus en plus grands $^{29}$. De nouvelles approches innovantes et collaboratives sont donc nécessaires pour résoudre ce qui est souvent qualifié de « problème complexe ${ }^{30}$.

\subsection{Innovation, capacités dynamiques et écosystème}

L'innovation peut être définie comme un processus micro et macro dynamique par lequel des agents, des organisations, des institutions et des structures macro de l'économie sont transformés par les effets d'une idée nouvelle ${ }^{31}$. L'innovation peut aider les OSP à s'adapter à l'environnement complexe et changeant dans lequel elles évoluent. En effet, il a été

\footnotetext{
${ }^{28}$ Plus amples explications théoriques en lien avec les concepts d'IO et d'écosystème d'innovation sont présentées à l'annexe 1.

29 (De Vries et al. 2016; Koppenjan and Klijn 2004; Mergel 2015; Mergel 2018).

30 "Wicked problems" (Edmondson 2016; Sørensen and Torfing 2011).

31 Traduction libre de " a micro and macro dynamic process by which agents, organizations, institutions and the macro structure of the economy are transformed by the effects of a novel idea" (Potts and Kastelle 2010, p. 123).
} 
Pour être innovantes, les OSP doivent donc revoir

leurs structures

organisationnelles et leurs

modes d'interactions.

démontré que l'innovation, dans le secteur public, contribue à l'amélioration de la qualité, de l'efficacité et de l'efficience des services publics, à l'augmentation de la satisfaction et de la confiance des citoyens ainsi qu'à l'implication des citoyens et des partenaires privés ${ }^{32}$.

Pour être innovantes, les OSP doivent donc revoir leurs structures organisationnelles et leurs modes d'interactions. Plusieurs propositions ont été formulées pour améliorer les capacités d'innovation des OSP comme, par exemple, la déréglementation, la décentralisation des pouvoirs décisionnels, la révision des indicateurs de performance, la modification des systèmes de rémunération et de bonification, la privatisation, le recrutement d'innovateurs reconnus ou encore la mise en place de conditions favorables où les idées novatrices peuvent se développer. Des recherches ont également proposé diverses stratégies d'ouverture et de collaboration, telles que les réseaux de créativité, le crowdsourcing ou l'innovation axée sur l'utilisateur ${ }^{33}$. De telles stratégies sont liées à l'approche d'innovation ouverte (IO) qui focalise sur « les apports et les sorties de connaissances afin, à la fois, d'accélérer l'innovation interne et d'élargir les marchés pour l'utilisation externe de l'innovation ${ }^{34}$.

Huizingh (2011) soutient que " I'IO oblige les gestionnaires à prendre de nouvelles décisions dans le développement et l'exploitation des activités d'innovation. Quand, comment, avec qui, dans quel but et de quelle manière devraient-ils coopérer avec des parties extérieures ${ }^{35}$. Ainsi, afin de maximiser les bénéfices de I'IO, les OSP doivent développer des capacités organisationnelles et déployer des pratiques/actions

\footnotetext{
32 (Bekkers et al. 2011; Damanpour and Schneider 2009; De Vries et al. 2016; Green et al. 2014; Koppenjan and Klijn 2004; Linders 2012)

33 (Eggers and Singh 2009; Hartley et al. 2013; Meijer 2019; Potts and Kastelle 2010; Sørensen and Torfing 2011; Stewart-Weeks and Kastelle 2015)

34 Traduction libre: "inflows and outflows of knowledge to accelerate internal innovation, and expand the markets for external use of innovation, respectively" (Eggers and Singh 2009, p. 1).

35 Traduction libre : " open innovation requires managers to make new decisions in developing and exploiting innovation activities. When, how, with whom, with what purpose, and in what way should they cooperate with outside parties".
} 
Les capacités dynamiques sont définies comme «la capacité d'intégrer, de développer et de reconfigurer des compétences internes et externes pour faire face à des environnements en évolution rapide ". leur permettant d'initier et d'exploiter ces initiatives d'IO de manière durable ${ }^{36}$. Or, l'étude des 10 dans le secteur public, en particulier en lien avec les capacités organisationnelles et surtout les pratiques/actions nécessaires pour déployer des initiatives d'IO, est mal comprise et peu implantée dans le secteur public.

La présente recherche vise donc à combler ce vide en adoptant la perspective des capacités dynamiques pour étudier les pratiques/actions organisationnelles que les OSP devraient mettre en œuvre afin de s'engager avec succès dans des initiatives d'IO en contexte d'écosystème d'innovation. Les capacités dynamiques sont définies comme "la capacité d'intégrer, de développer et de reconfigurer des compétences internes et externes pour faire face à des environnements en évolution rapide ${ }^{37}$. La notion de compétence est formée de trois composantes clés, soit 1) les connaissances, c.-à-d. les savoirs, 2) les habiletés, c.-à-d. les savoir-faire et, 3) les attitudes, c.-à-d. les savoir-être.

Les capacités dynamiques sont mises en œuvre par le déploiement d'un ensemble de pratiques/actions organisationnelles qui se combinent et se complètent afin de faciliter les changements organisationnels ${ }^{38}$ au niveau des savoirs, savoir-faire et des savoir-être. Les capacités dynamiques peuvent être distinguées des capacités opérationnelles ou "ordinaires", qui se rapportent aux opérations courantes d'une organisation. Les capacités dynamiques, en revanche, désignent «la capacité d'une organisation à créer, étendre ou modifier délibérément sa base de ressources ${ }^{39}$. L'hypothèse de base du cadre des capacités dynamiques est que les compétences de base devraient être utilisées pour modifier les positions " concurrentielles " à court terme qui peuvent être utilisées pour créer un avantage

\footnotetext{
${ }^{36}$ (Cheng and Huizingh 2015)

37 Traduction libre : " the ability to integrate, build, and reconfigure internal and external competencies to address rapidly-changing environments" (Teece et al. 1997, p. 517).

38 (Schilke et al. 2018; Yeow et al. 2018)

39 (Helfat et al. 2007)
} 
Les OSP doivent donc se concentrer sur la

"création "

d'écosystèmes

d'innovation qui devraient permettre à différents acteurs d'interagir afin de faire émerger les meilleurs idées et solutions possibles
« concurrentiel » à plus long terme. Les capacités dynamiques offrent donc une lentille utile pour mieux comprendre comment une OSP peut transformer sa stratégie, ses ressources existantes, ainsi que l'ensemble de l'écosystème dans lequel elle évolue. Notre étude se concentre donc sur la mise en œuvre d'initiatives " exemplaires ${ }^{40} d^{\prime} I O$, qui ont été qualifiées de succès, dans un contexte d'écosystème d'innovation.

\section{Écosystème d'innovation : Mise en contexte et définition}

De nos jours, le concept d'écosystème d'innovation est un sujet de préoccupations de plus en plus important, aussi bien pour les gestionnaires d'organisations privées que d'OSP, et pour cause $^{41}$. Effectivement, l'accroissement du niveau d'interdépendances entre les organisations et le rythme de plus en plus rapide des changements aussi bien technologiques, sociaux, politiques, environnementaux, légaux ou d'affaires, signifient que la création de valeur et la prise de décision peuvent être difficilement centralisées entre les mains d'une seule organisation. Ce constat est particulièrement marqué en matière d'innovations et particulièrement en matière $d^{\prime} 1 \mathrm{O}^{42}$. Les organisations, privées comme publiques, et leurs gestionnaires doivent comprendre et accepter qu'il sera de plus en plus difficile de générer "seul " les meilleures idées, les meilleures innovations. De plus, compte tenu du fait que les organisations sont confrontées à des situations problématiques de plus en plus volatiles, incertaines, complexes et ambiguës, et qu'elles ne possèdent généralement pas toutes les ressources et compétences nécessaires pour développer et déployer des innovations pour surmonter ces problèmes ${ }^{43}$, elles doivent collaborer avec d'autres organisations. Cette collaboration passe, entre autres, par des initiatives d'IO.

\footnotetext{
${ }^{40}$ Source: https://oecd-opsi.org/our-work/case-studies/

${ }^{41}$ (Jacobides et al. 2018; Oh et al. 2016)

42 (Adner 2006; Adner and Euchner 2014)

43 (Talmar et al. 2020)
} 
Les OSP doivent donc se concentrer sur la "création » d'écosystèmes d'innovation qui devraient permettre à différents acteurs, comme, par exemple, des organisations privées, des entrepreneurs, des investisseurs, des chercheurs, des utilisateurs, des communautés, des consortiums, des décideurs politiques, des gouvernements, des organisations sociales, etc. ${ }^{44}$, d'interagir afin de faire émerger les meilleures idées et solutions possibles ${ }^{45}$. Un écosystème d'innovation représente un "ensemble évolutif d'acteurs, d'activités et d'artefacts, ainsi que les institutions et les relations, y compris les relations de complémentarité et de substitution, qui sont importants pour la performance d'innovation d'un acteur ou d'une population d'acteurs ${ }^{46}$.

\subsection{Innovation et innovation ouverte dans le secteur public}

Outre la perspective de meilleurs résultats d'innovations et la création de valeur, les initiatives d'IO en contexte d'écosystème

Les initiatives d'IO peuvent permettre aux OSP d'accroître leur niveau de transparence et de responsabilisation, améliorer la prise de décision ainsi qu'accroître le bien-être des citoyens. d'innovation peuvent permettre aux OSP d'accroitre leur niveau de transparence et de responsabilisation, améliorer la prise de décision ainsi qu'accroître le bien-être des citoyens. Les approches d'IO peuvent aider les OSP à repousser leurs limites afin de saisir et d'intégrer les connaissances et les idées de contributeurs externes (p. ex. citoyens, groupes communautaires, entreprises privées) qui ne sont traditionnellement pas impliquées dans de tels processus d'innovation ${ }^{47}$.

La diversité des contributeurs ainsi que la capture de connaissances et d'idées externes obligent les OSP à faire des choix complexes. En particulier, les OSP doivent sélectionner

\footnotetext{
44 (Tsujimoto et al. 2018)

45 (Osterwalder et al. 2019)

46 « An innovation ecosystem is the evolving set of actors, activities, and artifacts, and the institutions and relations, including complementary and substitute relations, that are important for the innovative performance of an actor or a population of actors (p. 1) » (Granstrand and Holgersson 2020).

47 (Bommert 2010; Hilgers and Ihl 2010)
} 
Plusieurs facteurs pouvant faciliter ou encore entraver la mise en ceuvre de l'IO dans le secteur public ont été identifiés. des approches de participation appropriées le long d'un continuum qui s'étend d'une production participative générale (crowdsourcing) à une production participative ciblée avec des partenaires identifiés pour effectuer des tâches spécifiques ${ }^{48}$.

Chaque approche de participation a non seulement ses avantages et ses opportunités, mais aussi ses propres défis qui nécessitent le déploiement de ressources et d'infrastructures spécifiques. Par exemple, l'approche de production participative générale peut impliquer un groupe important et anonyme de personnes, généralement des citoyens dans le contexte d'OSP, qui contribuent à la création d'un bien public en répondant à un appel à contributions ouvert ${ }^{49}$. En comparaison, le mode de coproduction ciblé offre une forme d'IO plus intégrée et étroitement collaborative dans laquelle les citoyens et les fonctionnaires coopèrent dans les processus administratifs et d'élaboration des politiques.

À cette fin, les OSP peuvent utiliser les idées et les connaissances des contributeurs en organisant des activités de coproduction, par exemple, des compétitions d'innovation telles que des marathons d'innovation (hackathons), ou des évènements de démarrage d'entreprise (startup weekends). Les OSP peuvent également favoriser "l'autoapprovisionnement » de services par les citoyens grâce à l'installation d'infrastructures appropriées, telles que des portails communautaires pour la prise de décision collaborative ou encore la mise sur pied de plateformes de données ouvertes ${ }^{50}$.

Plusieurs facteurs pouvant faciliter ou encore entraver la mise en œuvre de I'IO dans le secteur public ont été identifiés. Au niveau organisationnel, le niveau de disponibilité des ressources, le degré de centralisation du pouvoir décisionnel, les styles de leadership, le niveau de tolérance à l'erreur, la culture d'innovation, le degré d'aversion au risque, ainsi que les

\footnotetext{
48 (Mergel 2015, p. 601).

49 (Linders 2012; Mergel 2015)

50 (Linders 2012)
} 
Les capacités dynamiques peuvent être distinguées selon la capacité à :

1) Détecter les opportunités / menaces émergentes dans son environnement,

2) Saisir les opportunités et éviter les menaces en évaluant les avantages / risques, et

\section{3) Mobiliser les actifs /} capacités nécessaires pour y répondre rapidement incitatifs et les récompenses, sont des facteurs influençant I'innovation dans le secteur public.

Au niveau individuel, l'autonomisation des employés et des citoyens, les connaissances et compétences liées aux $\mathrm{Tl}$ et la créativité ont été identifiées comme d'importants antécédents de l'innovation. Les investissements en TI jouent également un rôle clé dans les initiatives d'IO en favorisant, par exemple, la participation citoyenne, la mise en commun d'idée ou encore l'accès à des banques de données. Poussées par la convergence des Tl et des réseaux sociaux, les OSP peuvent accéder à des bases de données et de connaissances vastes et diverses pouvant les aider à définir et à résoudre plus efficacement des problèmes auxquels elles sont confrontées. À leur tour, les citoyens, disposant d'un accès universel et numérique à l'information et aux services, peuvent non seulement contribuer plus efficacement à la sphère publique, mais aussi forcer les OSP à plus de transparence et de responsabilisation, ce qui peut se traduire par une confiance accrue dans les institutions publiques ${ }^{51}$.

\subsection{Capacité dynamique, innovation ouverte et écosystème}

Tel que définie précédemment, la notion de capacités dynamiques réfère à la capacité d'une organisation à intégrer, développer et reconfigurer des compétences, aussi bien internes et externes, afin d'évaluer et s'adapter à son environnement ${ }^{52}$. Ces capacités sont spécifiques au contexte de chaque organisation et leur développement doit être fait au fil du temps et nécessite d'importants investissements en temps, en efforts et en ressources financières ${ }^{53}$. Ainsi, les capacités dynamiques d'une organisation peuvent être distinguées selon la capacité à :

\footnotetext{
51 (Borins 2001; Hilgers and Ihl 2010)

52 (Teece et al. 1997, p. 516).

53 (Helfat and Peteraf 2003; Schilke et al. 2018; Wernerfelt 1984)
} 
1) Détecter les opportunités/menaces émergentes dans son environnement,

2) Saisir les opportunités et éviter les menaces en évaluant les avantages/risques, et

3) Mobiliser les actifs/capacités nécessaires pour y répondre rapidement et de manière appropriée en améliorant, combinant, protégeant et, si nécessaire, reconfigurant ses actifs tangibles. ${ }^{54}$

Ainsi, pour développer sa capacité à détecter les opportunités et les menaces, une OSP doit développer une compréhension globale de son environnement en veillant, recherchant et explorant constamment l'évolution des besoins/exigences des citoyens, des groupes et des organisations sous sa responsabilité; les changements politiques, économiques, sociaux, environnementaux et légaux à la fois locaux et internationaux; ainsi que les évolutions et innovations technologiques au niveau local, mais également dans d'autres juridictions, c.-à-d. la Détection.

Par la suite, une fois qu'une nouvelle idée, une opportunité ou encore une menace est détectée, une OSP doit faire des choix stratégiques et investir du temps, des efforts et des fonds pour saisir l'opportunité ou éviter une menace potentielle, c.-à-d. la Capture. Cependant, saisir une opportunité signifie également que l'OSP doit développer ses capacités à reconfigurer ses actifs, ses ressources et sa structure, c.-à-d. la Transformation.

Ces trois types de capacités posent des défis aux OSP désirant mettre en œuvre des initiatives d'IO dans un contexte d'écosystème d'innovation. Par exemple, un recours aux contributions citoyennes peut permettre d'obtenir une grande quantité d'idées et de propositions (détection). Par contre, passer en revue toutes ces idées, les trier et sélectionner la ou les plus porteuses, représentent souvent un casse-tête pour les OSP, en plus de nécessiter énormément de ressources (capture). De plus, la plupart des OSP vont investir beaucoup de

54 (Teece et al. 2016; Teece et al. 1997) 
temps et de ressources afin d'impliquer des contributeurs externes pour générer des idées, mais, parallèlement, investissent très peu dans la sélection, la mise en œuvre et la diffusion des innovations (transformation) ${ }^{55}$.

Afin de mieux comprendre comment ces trois types de capacités sont déployées par des OSP ainsi que de guider les décideurs d'OSP souhaitant développer les capacités dynamiques permettant de lancer et de supporter des initiatives d'IO, une recension des défis et des bonnes pratiques, c.-à-d. des actions organisationnelles, a été effectuée.

55 (Eggers and Singh 2009) 
Dans un contexte de complexité croissante, d'évolution rapide des demandes et de pressions budgétaires considérables, les OSP doivent comprendre, tester et intégrer de nouvelles façons de faire les choses

\section{Recension de cas " exemplaires " d'innovations ouvertes dans un contexte d'écosystème d'innovation ${ }^{56}$}

Le déploiement d'initiatives d'innovations ouvertes (IO) dans le secteur public pose un défi particulier pour les gestionnaires. Or, puisque l'étude de ce phénomène, qui est relativement mal compris et peu implanté dans le secteur public, est limitée, la présente étude a identifié, à partir d'études de cas impliquant des OSP, des:

1) Défis liés au déploiement et maintien d'IO dans un contexte d'écosystème d'innovation, et

2) Bonnes pratiques/actions organisationnelles déployées par les OSP afin d'accroître le succès d'initiatives d'IO.

Pour ce faire, des études de cas d'IO déployées dans des OSP en contexte d'écosystème d'innovation, et ayant été des succès, ont été analysées. Au total, ce sont 253 initiatives d'IO ${ }^{57}$, lancées entre 2009 et 2020, qui ont été identifiées et sélectionnées à partir des cas " exemplaires » documentés par I'Observatoire des Innovations du Secteur Public (OPSI) (https://oecd-opsi.org/our-work/case-studies/). L'OPSI est un forum mondial pour l'innovation dans le secteur public et chapeauté par l'Organisation de Coopération et de Développement Économiques (OCDE).

Dans un contexte de complexité croissante, d'évolution rapide des demandes et de pressions budgétaires considérables, les OSP doivent comprendre, tester et intégrer de nouvelles façons de faire les choses. L'OPSI collabore avec les OSP afin de comprendre et d'encourager de nouvelles approches pour résoudre les problèmes complexes de la société en donnant aux décideurs et aux fonctionnaires de nouvelles perspectives, connaissances, outils et connexions pour les aider à explorer de

\footnotetext{
56 L'annexe 2 présente une description de la recension des cas analysés ainsi qu'une liste des documents identifiés et consultés pour la rédaction du présent rapport.

57 Voir annexe 2 pour détails
} 
nouvelles possibilités. L'OPSI documente et diffuse des études de cas " exemplaires" afin que l'ensemble des OSP puissent voir ce que d'autres innovateurs œuvrant dans des OSP font au niveau international, tirer des leçons de leur expérience et prendre contact avec les personnes concernées pour poser des questions ou collaborer. Les résultats de l'analyse de ces études de cas sont présentés dans les prochaines sections.

\subsection{Défis reliés au déploiement d'initiatives d'IO menées par des OSP}

Au total, ce sont 17 défis reliés au déploiement d'IO par des OSP

17 défis reliés au déploiement d'IO par des OSP qui ont été identifiés et regroupés dans 4 catégories et sont décrits ci-dessous :

1. Collaboration interorganisationnelle

2. Processus d'innovation en contexte OSP

3. Implication des citoyens

4. Données et technologie qui ont été identifiés. Ces défis ont été regroupés dans 4 catégories décrites ci-dessous :

1. Collaboration interorganisationnelle

2. Processus d'innovation en contexte OSP

3. Implication des citoyens

4. Données et technologie

\section{Catégorie \#1 - Défis liés à la Collaboration} interorganisationnelle

Suite à l'intérêt initial envers le lancement d'une initiative d'IO, la majorité des OSP doivent s'assurer de maintenir l'implication et l'engagement des différents partenaires impliqués tout au long de l'initiative. Cette implication nécessite des efforts, du temps, de l'énergie ainsi que la mobilisation de ressources et d'expertises. L'objectif pour les OSP est d'éviter un désintérêt envers l'initiative à travers le temps ainsi qu' une diminution de l'effort fourni. Le premier défi est donc de maintenir l'implication et l'engagement des partenaires tout au long de l'initiative d'IO (D1).

Généralement, les initiatives d'IO chapeautées par des OSP favorisent et encouragent la participation d'un nombre important et varié de partenaires impliqués. Or, il devient essentiel d'utiliser et/ou développer des approches, des 
méthodologies, des processus et des outils collaboratifs communs à l'ensemble des partenaires impliqués dans une initiative d'IO. Généralement, chaque partenaire utilise ses propres outils et possède ses propres pratiques de collaboration. Par ailleurs, lors d'une initiative d'IO, les OSP doivent s'assurer qu'un langage commun sera établi et que des pratiques, des cadres, des outils et des processus collaboratifs communs seront adoptés et utilisés. Un deuxième défi est donc de développer un langage commun et d'utiliser des outils collaboratifs communs à l'ensemble des partenaires impliqués dans une initiative d'IO (D2).

Afin d'accroître les probabilités de succès des initiatives d'IO, il est essentiel que les OSP développent une vision commune de I'initiative afin d'aligner les objectifs, les attentes et les efforts de chacun des partenaires impliqués. Les partenaires impliqués ont parfois des attentes et des intérêts divergents qui peuvent venir affecter le déroulement et les dynamiques des initiatives d'IO. Le défi pour les OSP est donc d'aligner ces intérêts et les efforts de chacun en écoutant, en négociant, en faisant des compromis et en maintenant une vision claire du projet. Un troisième défi est de développer une vision commune afin d'aligner les partenaires impliqués (D3).

Afin de favoriser les initiatives d'IO, les OSP doivent tenter de rompre les silos et les frontières ( $p$. ex. entre départements, entre organisations, entre groupes d'appartenances / identitaires) afin de favoriser une circulation et un partage fluide et ouvert des informations, des connaissances et des idées. Pour les OSP, il s'agit de trouver les canaux de communication adéquats, d'établir des pratiques claires et communes de collaboration ainsi que d'en assurer l'acceptation par l'ensemble des partenaires impliqués. Un quatrième défi est de favoriser le partage fluide de l'information, des connaissances et des idées (D4).

Par ailleurs, la réussite d'initiatives d'IO passe généralement par l'établissement d'un climat favorisant: 1) le partage d'informations et de données parfois sensibles, 2) la transparence, 3) la collaboration et 4) l'échange d'idées entre 
des partenaires impliqués qui ont une expérience et des objectifs souvent divergents. Un cinquième défi est donc d'établir un climat de confiance et de respect entre les partenaires impliqués (D5).

\section{Catégorie \#2 - Défis liés aux Processus d'innovation en contexte d'OSP}

Lors d'initiatives d'IO, il arrive régulièrement que ces initiatives soient confrontées, aussi bien à un manque de connaissances et d'expertise, qu'à un manque de ressources techniques et/ou financières. Les OSP doivent donc s'assurer, avant de se lancer dans une telle initiative, d'identifier les ressources et expertises clés à la réalisation et au succès de l'initiative en plus de s'assurer de leur accessibilité et disponibilité. Un sixième défi est d'identifier et d'obtenir les ressources et expertises (D6).

Les structures, les processus et les cadres de gouvernance existant dans les OSP sont souvent peu flexibles, hiérarchiques, bureaucratiques, en silo, conservateurs, lents et fastidieux. Ces éléments peuvent venir retarder et entraver la réalisation d'initiatives d'IO. II est donc impératif d'identifier les éléments de ces structures, processus et cadres qui influenceront les initiatives d'IO et de comprendre comment les contourner ou encore les utiliser pour accroître les probabilités de réussite. Un septième défi est d'identifier et surmonter les freins/lourdeurs structurelles et processuelles (D7).

Les OSP sont souvent caractérisées par de nombreuses normes, règlements, lois et procédures que les partenaires impliqués lors d'une initiative $d^{\prime} I O$ se doivent de comprendre et de respecter. Or, connaître, comprendre et respecter ces éléments peut demander beaucoup de temps et d'efforts, ce qui a généralement pour effet de ralentir le processus d'innovation et d'effriter le niveau d'engagement de certains partenaires impliqués. Un huitième défi est d'aider à faire comprendre et respecter rapidement et efficacement les normes, les procédures et les lois des OSP (D8). 
Les extrants d'initiatives d'IO peuvent entraîner un certain nombre de changements, de transformations au sein des OSP, des partenaires et/ou des futurs utilisateurs de l'innovation. Ainsi, afin d'accroître les probabilités de succès des IO, les OSP se doivent de faciliter le déploiement de ces transformations et faire accepter ces changements. Un neuvième défi est donc de gérer la résistance au changement engendrée par les 10 et leur extrants (D9).

Le temps alloué pour mener à terme des initiatives d'IO est souvent court afin d'obtenir rapidement des retombées. Par contre, cette pression " temporelle " peut engendrer des effets néfastes sur les initiatives d'IO ainsi que sur les relations et la collaboration entre les partenaires impliqués. En effet, la pression et la "longueur » du temps peut varier que l'on soit une OSP, une entreprise privée ou du milieu académique. Les entreprises privées fonctionnent généralement avec un "temps court" alors que, par exemple, les milieux académiques ont une réalité temporelle qualifiée de "temps long ". Un dixième défi est de respecter les réalités temporelles de chacun des partenaires impliqués dans l'initiative d'IO (D10).

Le succès d'initiatives $d^{\prime} I O$ repose généralement sur le fait d'obtenir l'appui et le support aussi bien des dirigeants et fonctionnaires des OSP que de leurs alliés stratégiques. Ces appuis et ce support permettent de bénéficier d'une marge de manœuvre nécessaire à la réalisation et au succès d'une initiative d'IO. Un onzième défi est d'obtenir un support stratégique de la direction et des alliés stratégiques (D11).

Dans certaines initiatives $d^{\prime} I O$, des intérêts politiques importants peuvent venir influencer le processus d'innovation. Dans d'autres cas, des changements politiques ( $p$. ex. nouveau gouvernement, nouvelles lois) peuvent réorienter les priorités et les objectifs de certaines initiatives d'IO. Un onzième défi est de prendre en compte et anticiper les enjeux politiques afin d'apporter les ajustements nécessaires (D12). 


\section{Catégorie \#3 - Défis liés à l'Implication des citoyens}

Être capable de comprendre les particularités des citoyens visés par une initiative d'IO et de s'y adapter sont essentiels aux succès mêmes d'une telle initiative. Par exemple, certaines personnes peuvent avoir de la difficulté à lire et à écrire ou encore à utiliser des appareils électroniques. La culture, leurs valeurs et croyances peuvent également être un frein à leur participation. Le défi est particulièrement important quand l'ensemble de la population est visé. Par ailleurs, certains groupes sont plus difficiles à rejoindre que d'autres, p. ex. les immigrants, les personnes moins éduquées, les habitants éloignés, les communautés rurales, les personnes âgées, etc. Un treizième défi est de bien comprendre et connaître les particularités et le contexte des citoyens afin d'accroître l'inclusion sociale (D13).

L'un des défis pour les OSP responsables d'initiative d'IO est d'identifier les canaux de communication les mieux adaptés afin de/d': 1) Faire connaître et publiciser une initiative d'IO auprès des citoyens; 2 ) Maintenir ces derniers informés sur l'état d'avancement de l'initiative et 3 ) Encourager les citoyens à participer. II est essentiel pour les OSP d'utiliser les moyens de communication permettant de joindre l'ensemble des citoyens touchés de près ou de loin par une initiative d'IO afin d'accroître les probabilités de succès. Certains outils utilisés, tels que les réseaux sociaux ou encore les messageries instantanées, ne sont pas accessibles par tous. Un quatorzième défi est d'identifier et d'utiliser les canaux de communication les mieux adaptés aux citoyens (D14).

Finalement, un défi majeur pour les OSP est d'obtenir l'engagement des citoyens et de le maintenir tout au long d'une initiative d'IO. Malgré un intérêt pour une initiative d'IO, les citoyens sont parfois réticents à participer et cela nécessite un effort pour les convaincre. Certaines catégories de personnes sont également plus difficiles à convaincre que d'autres, et ce, pour différents motifs (p. ex., vulnérabilité, culture, éducation, 
religion, etc.). Un quinzième défi est d'obtenir et maintenir l'engagement des citoyens participants (D15).

\section{Catégorie \#4 - Défis liés aux Données et technologie.}

Lors d'initiatives d'IO, I'infrastructure technologique existante est parfois un frein à l'innovation lorsqu'elle est inflexible et nécessite des transformations. Dans certains cas, le volume de données à traiter est trop important et l'infrastructure n'est pas adéquate. Dans d'autres cas, l'infrastructure technologique ne permet pas de supporter la collaboration entre les différents partenaires impliqués dans une initiative d'IO ou encore un accès limité à internet dans certains endroits complique le déploiement de l'initiative et/ou l'implantation de l'innovation. Un seizième défi est de surmonter les limites des infrastructures technologiques existantes (D16).

Finalement, les initiatives d'IO sont souvent confrontées à un volume important de données. Le défi est tout d'abord d'être capable de les rassembler sachant que différents partenaires impliqués ont différents types de données et qu'il existe des défis/problèmes de droit d'accès aux données, de transfert de données entre systèmes (format), de propriété ou encore de qualité de données. $D^{\prime}$ autre part, la sécurité des données est un enjeu crucial pour les OSP et les initiatives d'IO font face à de nombreux défis reliés à ces enjeux dans un contexte de collaboration avec différents partenaires impliqués (p.ex. que peut-on partager, avec qui, comment garantir la protection de ces données, etc.). Un dix-septième défi est d'assurer une gouvernance sécuritaire des données (acquisition, stockage, partage et sécurité) (D17).

Le tableau 1 présente un résumé des 17 défis décrits ci-dessus ainsi que le nombre de cas, dans notre échantillon, décrivant ces défis. 


\begin{tabular}{|c|c|c|}
\hline D\# & Libellés & Nb. cas \\
\hline \multicolumn{3}{|c|}{ COLLABORATION INTER-ORGANISATIONNELLE } \\
\hline D1 & $\begin{array}{l}\text { Maintenir l'implication des partenaires tout au long de l'initiative de } \\
\text { IO }\end{array}$ & 37 \\
\hline D2 & $\begin{array}{l}\text { Développer un langage commun et utiliser des outils collaboratifs } \\
\text { communs à l'ensemble des partenaires impliqués }\end{array}$ & 22 \\
\hline D3 & $\begin{array}{l}\text { Développer une vision commune afin d'aligner les partenaires } \\
\text { impliqués }\end{array}$ & 21 \\
\hline D4 & Partage fluide de l'information, des connaissances et des idées & 16 \\
\hline D5 & $\begin{array}{l}\text { Établir un climat de confiance et de respect entre les partenaires } \\
\text { impliqués }\end{array}$ & 13 \\
\hline \multicolumn{3}{|c|}{ PROCESSUS D'INNOVATION EN CONTEXTE d'OSP } \\
\hline D6 & $\begin{array}{l}\text { Identifier et obtenir les ressources et expertises nécessaires à la } \\
\text { réalisation l'initiative d'IO }\end{array}$ & 44 \\
\hline D7 & $\begin{array}{l}\text { Identifier et surmonter les freins/lourdeurs structurels et } \\
\text { processuels }\end{array}$ & 25 \\
\hline D8 & $\begin{array}{l}\text { Faire comprendre et respecter rapidement et efficacement les } \\
\text { normes, les procédures et les lois des OSP }\end{array}$ & 24 \\
\hline D9 & $\begin{array}{l}\text { Gérer la résistance au changement engendrée par les } 10 \text { et leurs } \\
\text { extrants }\end{array}$ & 14 \\
\hline D10 & $\begin{array}{l}\text { Respecter les réalités temporelles de chacun des partenaires } \\
\text { impliqués dans une initiative d'IO }\end{array}$ & 12 \\
\hline D11 & $\begin{array}{l}\text { Obtenir un support stratégique de la direction et des alliés } \\
\text { stratégiques }\end{array}$ & 11 \\
\hline D12 & $\begin{array}{l}\text { Prendre en compte et anticiper les enjeux politiques afin d'apporter } \\
\text { les ajustements nécessaires }\end{array}$ & 8 \\
\hline \multicolumn{3}{|c|}{ IMPLICATION DES CITOYENS } \\
\hline D13 & $\begin{array}{l}\text { Bien comprendre et connaître les particularités et le contexte des } \\
\text { citoyens afin d'accroître l'inclusion sociale }\end{array}$ & 23 \\
\hline D14 & $\begin{array}{l}\text { Identifier et utiliser les canaux de communication adaptés aux } \\
\text { citoyens }\end{array}$ & 17 \\
\hline D15 & Obtenir et maintenir l'engagement des citoyens participants & 17 \\
\hline \multicolumn{3}{|c|}{ DONNÉES ET TECHNOLOGIES } \\
\hline D16 & Surmonter les limites des infrastructures technologiques existantes & 38 \\
\hline D17 & $\begin{array}{l}\text { Assurer une gouvernance sécuritaire des données (acquisition, } \\
\text { stockage, partage) }\end{array}$ & 35 \\
\hline
\end{tabular}




\subsection{Bonnes pratiques/actions organisationnelles déployées par les OSP lors d'initiatives d'IO}

$\mathrm{Au}$ total, 18 bonnes pratiques/actions organisationnelles déployées par les OSP pour supporter la mise en œuvre $d^{\prime}$ 'initiatives d'IO ont été identifiées. Ces pratiques/actions sont décrites dans les paragraphes qui suivent.

Le tableau 2 présente la liste synthèse de ces 18 pratiques/actions en plus de les caractériser selon le type de capacité dynamique auquel chaque pratique/action contribue. Il est important de rappeler que c'est par l'entremise du déploiement de ces pratiques/actions organisationnelles que les capacités dynamiques de détection (De.), de capture (Ca.) et de transformation (Tr.), liées aux initiatives d'IO impliquant des OSP, sont mises en place. Chaque pratique/action organisationnelle peut refléter et être associée à plus d'un des trois types de capacités dynamiques (voir tableau 2). Ces pratiques/actions ont été mises en œuvre et utilisées collectivement pour réaliser les initiatives d'IO dans les cas exemplaires d'OSP étudiés.

\section{Bonne pratique \#1 \\ Favoriser une culture d'ouverture et d'expérimentation}

La première bonne pratique identifiée afin d'accroître les probabilités de succès $d^{\prime} I O$ dans les OSP est de favoriser une culture organisationnelle où l'expérimentation et l'apprentissage expérientiel sont encouragés. II s'agit de favoriser, aussi bien dans les OSP que dans les initiatives d'IO, un environnement où la patience, le support et la prise de risques sont énoncés, cultivés et acceptés. Dans cet environnement d'expérimentation et d'ouverture, les échecs sont acceptés/tolérés, car ils peuvent servir de sources d'apprentissage importantes. Par la suite, ces apprentissages sont partagés dans les OSP, mais aussi avec les partenaires des 
OSP. Une communication ouverte et la transparence sont également des caractéristiques essentielles, afin que tous les partenaires impliqués dans une initiative d'IO se sentent libres de signaler les écueils, les problèmes et les échecs. De plus, tous les partenaires impliqués devraient également être disposés à prendre en considération, à évaluer et à déployer des idées même si elles viennent de l'extérieur de leurs organisations.

\section{Bonne pratique \#2 Impliquer les partenaires à l'aide de plateformes collaboratives et coordonner les échanges}

Utiliser des plateformes collaboratives pour coordonner les efforts des partenaires impliqués dans l'ensemble des phases d'une initiative d'IO est crucial. En effet, les partenaires internes aussi bien qu'externes doivent être autorisés à participer ouvertement et en toute transparence sur les plateformes collaboratives déployées pour supporter les initiatives d'IO. Chaque partenaire impliqué peut y jouer des rôles clés. Ces plateformes doivent également favoriser l'engagement des participants, le partage de l'avancement du projet, la communication, l'échange d'informations, la génération et la sélection d'idées ainsi que la collaboration entre les partenaires impliqués. De plus, ces plateformes doivent faciliter la mise en commun de connaissances, de ressources, d'expériences, de réseaux et d'antécédents organisationnels diversifiés et complémentaires. Sur ces plateformes collaboratives, l'un des rôles clés souvent joué par les OSP est celui de coordonnateur. Effectivement, afin de favoriser l'engagement des partenaires et faciliter la coordination des échanges, des communications, des tâches ainsi que du partage des idées et des connaissances, les OSP doivent investir du temps et de l'énergie pour accomplir ce 
travail souvent invisible, mais essentiel au succès des initiatives d'IO.

\section{Bonne pratique \#3 \\ Générer et collecter des idées à l'aide d'une plateforme numérique}

En plus de favoriser la collaboration via une plateforme numérique, il est essentiel de déployer une plateforme numérique afin de permettre aux utilisateurs finaux, aux citoyens et aux différents partenaires impliqués de générer et proposer de nouvelles idées. Cette plateforme doit également permettre de fournir des commentaires et de la rétroaction sur les idées et innovations proposées et/ou en cours de développement. Par ailleurs, elle devrait favoriser des interactions ouvertes, transparentes et en toute confiance entre les différents partenaires impliqués. II est fréquent que, sur ce type de plateformes utilisées lors d'initiatives d'IO, les OSP soient appelées à jouer à la fois un rôle de modérateur au niveau des échanges et des idées, ainsi qu'un rôle de motivateur pour stimuler la génération et les échanges d'idées et de points de vue. Finalement, la plateforme devient un point central de collecte d'idées/commentaires et peut servir de base de connaissances.

\section{Bonne pratique \#4}

Développer une présence sur les réseaux sociaux

Lors d'initiatives d'IO impliquant des OSP, il est très important pour l'ensemble des partenaires impliqués, mais particulièrement pour les OSP, de développer une présence sur les réseaux sociaux pour favoriser les interactions entre les personnes internes et externes à l'organisation (citoyens, 
autres partenaires, etc.). Cette présence peut permettre de créer un momentum autour d'une initiative d'IO. Pour ce faire, les OSP et les partenaires doivent identifier le public cible et les canaux les plus efficaces pour les rejoindre (p. ex. Facebook, Instagram Twitter, etc.). La présence sur les réseaux sociaux permet également aux OSP d'identifier de nouvelles personnes ou partenaires qui peuvent soutenir l'effort d'innovation et/ou transmettre des informations pertinentes pour promouvoir les résultats de l'innovation.

\section{Bonne pratique \#5 Organiser des rencontres entre les partenaires clés impliqués dans l'IO}

Afin de favoriser la collaboration et de stimuler la génération et l'échange d'idées, il est important que les OSP prennent en charge, seules ou accompagnées, l'organisation de rencontres entre partenaires impliqués. Ces rencontres, virtuelles ou en présentiel, doivent avoir des thématiques variées et des objectifs clairs. Elles peuvent prendre différentes formes, telles que des hackathons, des coopérathons, des rallyes, des ateliers de design thinking ou d'autres types d'activités. Ces opportunités peuvent être utilisées pour inciter les partenaires à mieux se connaitre, pour transmettre des commentaires/suggestions sur le processus d'innovation, pour susciter de l'intérêt pour l'innovation, pour accéder à de nouveaux partenaires potentiels, pour favoriser l'émergence de communautés ou encore pour faciliter les interactions/échanges entre les partenaires impliqués. 


\section{Bonne pratique \#6 \\ Cartographier les besoins et les capacités des utilisateurs finaux}

Les initiatives d'IO impliquant des OSP sont généralement lancées afin de concevoir un produit, un service, un processus de fabrication ou un processus organisationnel afin de répondre aux besoins d'utilisateurs finaux comme, par exemple, des citoyens ou des organisations bénéficiaires. Ainsi, il devient primordial de cartographier les utilisateurs potentiels des probables innovations qui émergeront d'initiatives d'IO. Les utilisateurs peuvent être cartographiés en termes de connaissances, de capacités (par exemple, compétences techniques, connaissances informatiques), d'intérêts et de préférences (par exemple, confiance dans les services en ligne, etc.). Cela peut être fait en collaborant avec des partenaires impliqués qui travaillent avec les utilisateurs finaux ou encore directement avec les utilisateurs eux-mêmes. Les observations, les sondages, les communications en face-à-face ou en ligne, ou même l'ethnographie peuvent être utilisés pour identifier et comprendre les besoins des utilisateurs. L'objectif est de développer des innovations adaptées à leurs besoins et de favoriser l'adoption de l'innovation.

Bonne pratique \#7 Développer des prototypes et des prétests fréquemment

Il est très fréquent que, lors d'initiative d'IO, les partenaires impliqués adoptent une approche itérative lors de la conception et du développement d'innovations. Cette 
approche encourage le développement de prototypes et la réalisation de plusieurs tests de ces prototypes. Ces boucles d'itérations courtes, fréquentes et exigeant peu de ressources permettent de rapidement tester des hypothèses, obtenir de la rétroaction et apprendre de ces tests afin d'améliorer les innovations en développement. Idéalement, le développement et les tests de ces prototypes doivent être effectués, si possible, avec de vrais utilisateurs à différentes étapes du projet afin d'obtenir rapidement des commentaires et de pouvoir s'ajuster en conséquence. Les prototypes et les tests peuvent aider à sélectionner les technologies et fonctionnalités appropriées, à supporter une amélioration continue et à mieux répondre aux besoins des utilisateurs finaux.

\section{Bonne pratique \#8 Adopter une approche agile}

Chaque initiative d'IO peut être abordée par les décideurs et fonctionnaires d'OSP comme un projet, car, tout comme un projet, une initiative d'IO est mise sur pied pour " livrer " une ou des innovations uniques et sans précédent. De plus, elle regroupe des personnes et des partenaires qui travaillent ensemble temporairement, et ce, très souvent, pour la première fois. Ainsi, l'adoption d'une approche agile, qui met l'emphase sur la collaboration entre des équipes autoorganisées et pluridisciplinaires et leurs utilisateurs finaux, est tout à fait avisée lors d'initiatives d'IO. Une approche agile repose sur l'utilisation d'un cadre méthodologique léger, mais suffisamment centré sur l'humain et la communication. Elle préconise une planification adaptative, un développement évolutif, une livraison précoce et une amélioration continue. Adopter une approche agile devrait permettre d'avoir la capacité de s'améliorer continuellement, de livrer plus fréquemment et plus rapidement (pour améliorer le ratio time- 
to-market), de s'adapter rapidement aux changements, d'avoir une meilleure compréhension des besoins des utilisateurs et de s'adapter en permanence aux évolutions technologiques. Livrer plus fréquemment permet également de prendre connaissance des plaintes et réclamations des utilisateurs plus rapidement et de pouvoir les régler plus facilement et plus vite.

\section{Bonne pratique \#9}

\section{Gérer les enjeux relatifs à la gouvernance et à la sécurité des données}

De nos jours, l'utilisation de plateformes numériques est centrale pour le déroulement et la réussite des initiatives d'IO. Ces plateformes permettent de collaborer, communiquer, partager des idées et de conserver toutes les données générées et échangées. II est donc très important que les partenaires impliqués dans des initiatives d'IO, et tout particulièrement les OSP qui sont souvent responsables de la mise en place et la gestion de ces plateformes, portent une attention toute spéciale à la gouvernance et à la sécurité des données. Les règles d'accessibilité, de partage, de modification et d'utilisation des données doivent être clairement établies en amont du déploiement des plateformes numériques. Les OSP doivent, entre autres, considérer avec attention et gérer les aspects légaux liés à l'utilisation et à la propriété des données. La sécurité des données est aussi un enjeu majeur pour les OSP, qui peut entrainer des craintes et réticences de tous les partenaires impliqués. Des spécialistes en sécurité des données peuvent aider les OSP à bien comprendre les standards de sécurité, s'y conformer et/ou participer à l'évolution de ces standards. II est également important de partager, avec les différents partenaires, l'information relative à la sécurité des données afin de créer un climat de confiance. 


\section{Bonne pratique \#10 \\ Promouvoir une communication ouverte et transparente entre les partenaires impliqués}

Le développement d'un climat de collaboration basé sur la confiance est primordial pour le fonctionnement harmonieux et le succès d'initiatives $d^{\prime} I O$. Un des éléments qui devrait aider le développement et le maintien d'un climat de confiance est de promouvoir une communication ouverte et transparente. Cette responsabilité revient généralement aux OSP. Afin de favoriser une communication ouverte et transparente, il est important que les OSP rappellent aux partenaires impliqués des initiatives $d^{\prime} \mid 0$, de/d': 1) Reconnaître que les opinions/idées/points de vue de tous les partenaires sont importants, 2) Demander aux partenaires de partager leurs avis, 3) Écouter attentivement et de manière réfléchie l'ensemble des partenaires impliqués, 4) Engager les partenaires sur le plan personnel, 5) Être respectueux lors des échanges, 6) Rendre les informations et les résultats accessibles, 7) Reconnaître l'apport et les contributions de chaque partenaire, et 8) Prêcher par l'exemple. Cette communication transparente permet non seulement de faciliter le processus d'innovation en favorisant l'implication des partenaires, mais également de faciliter son implantation.

Bonne pratique \#11

Renforcer la motivation et l'engagement des

partenaires 
Renforcer la motivation et l'engagement des partenaires lors d'initiatives d'IO facilitera à la fois le déroulement des initiatives d'IO ainsi que la génération et le partage d'idées en plus d'accroître les probabilités de succès. Ce renforcement peut être réalisé en facilitant la collaboration, en augmentant la confiance des partenaires impliqués dans le processus d'innovation et en les responsabilisant. Les OSP peuvent également s'assurer de développer une vision claire de leurs initiatives d'IO afin de rallier et guider les partenaires impliqués. L'établissement d'objectifs communs, l'identification et la promotion des avantages de l'innovation ainsi que la clarification des gains/avantages de l'initiative d'IO pour chaque partenaire (p. ex. reconnaissance, récompenses monétaires, etc.) favoriseront également la motivation et l'engagement des partenaires impliqués.

\section{Bonne pratique \#12 Allouer des ressources financières suffisantes aux initiatives $d^{\prime} 10$}

Les initiatives d'IO sont similaires à des projets, car elles sont créées temporairement afin de produire une ou des innovations en mettant en commun les connaissances et les ressources de plusieurs partenaires impliqués. Or, tout comme dans un projet, l'aspect financier d'une initiative d'IO est essentiel au bon déroulement et aux probabilités de succès des projets. Il est donc très important qu'une OSP s'assure que, comme dans un projet, les ressources financières seront suffisantes tout au long des différentes phases d'une initiative d'IO. Un financement adéquat et anticipé permettra aux partenaires de se concentrer sur le cœur d'initiative $d^{\prime} I O$ soit la mise en commun de ressources/connaissances et la génération d'innovations. Dans certains cas, lorsque le financement est problématique, il peut être envisagé par les OSP d'obtenir du 
financement de sources alternatives, telles que du parrainage, du financement participatif, ou des accords avec des entreprises privées.

Bonne pratique \#13 Obtenir un support des hauts dirigeants

Lorsque des initiatives, peu importe leurs natures, sont lancées dans des organisations, l'un des facteurs clés de succès les plus importants, et qui perdurent à travers le temps, est l'obtention du support des hauts dirigeants. Ce facteur clé de succès est également essentiel au succès d'initiatives d'IO, car les hauts dirigeants ont plus d'influence sur la motivation de leurs subordonnés que quiconque dans une organisation et doivent communiquer dès le départ, aux employés et aux partenaires impliqués, les motifs et les objectifs des initiatives d'IO. Ainsi, l'obtention d'un soutien politique et de haut niveau, auprès des gouvernements, des agences et des partenaires et ce, à toutes les étapes du processus d'innovation, permettra: 1) de renforcer l'engagement des partenaires, 2) d'abattre les silos, 3) de promouvoir l'ouverture et le partage d'information et 4) de donner une impulsion au projet. Le soutien de la haute direction est également essentiel en amont d'initiative d'IO afin de libérer et d'obtenir les ressources nécessaires pour mener à bien ces initiatives.

Bonne pratique \#14 Développer un leadership fort et une vision claire et cohérente 
Deux éléments clés qui se cachent derrière les initiatives d'IO qui réussissent sont : 1) un leadership fort et 2) une vision claire et cohérente du processus d'innovation et des objectifs. Le leadership peut s'exprimer à travers une personne ou encore un groupe de personnes. Le ou les leaders sont responsables d'orchestrer, de fédérer et d'utiliser les énergies et les ressources individuelles et celles des partenaires impliqués pour la réalisation des objectifs communs d'une initiative d'IO. Dans un tel contexte, un leader doit inspirer et motiver les partenaires, doit faire preuve d'honnêteté et d'intégrité, doit résoudre les problèmes et répondre aux interrogations, doit communiquer largement et régulièrement avec tous les partenaires impliqués, doit mettre en avant les compétences et les ressources des partenaires, doit favoriser les échanges et doit anticiper les changements à venir. De plus, il est essentiel qu'une vision claire d'une initiative d'IO soit formulée afin de donner une direction aux partenaires impliqués. Celle-ci doit décrire le pourquoi et comment les objectifs d'une initiative seront atteints. Une vision permet d'avoir une orientation, d'inspirer et de motiver les participants, d'influencer l'engagement des partenaires, et de suivre et d'améliorer les processus d'innovation.

\section{Bonne pratique \#15 Identifier et sélectionner des membres d'équipe avec des compétences complémentaires et une bonne attitude}

Les initiatives $d^{\prime} I O$ nécessitent, tout au long du processus d'innovation, que des individus travaillent en équipe. Ces équipes sont généralement pluridisciplinaires et formées d'individus provenant ou associés aux différents partenaires impliqués. Or, l'identification et la sélection des individus qui formeront ces équipes doivent être faites judicieusement. 
Effectivement, les individus qui formeront des équipes pluridisciplinaires doivent idéalement posséder des connaissances et compétences complémentaires. II est important d'identifier des individus qui ont non seulement des compétences " techniques " complémentaires, mais aussi qui comprennent les besoins des utilisateurs (par exemple les citoyens) et qui y ont accès ou qui sont proches d'eux. L'autre élément auquel les OSP doivent faire particulièrement attention lors de la sélection des individus est de s'assurer que ces derniers ont une attitude d'ouverture, de respect, de curiosité, d'apprentissage, de créativité, d'écoute et qu'ils sont agiles.

\section{Bonne pratique \#16 Utiliser un cadre de travail collaboratif commun}

De nos jours, le travail collaboratif est utilisé lorsqu'un groupe d'individus souhaite atteindre un objectif commun et qu'il y a une interdépendance des tâches à effectuer par ces individus pour atteindre cet objectif. Le travail collaboratif permet, entre autres, de créer de la valeur, d'obtenir l'engagement des individus, de les responsabiliser, d'améliorer la résolution de problèmes, d'accroître la créativité et de générer plus d'innovation. Le travail collaboratif, qui est un moyen et non une fin, est donc au cœur même des initiatives d'IO. Or, le seul usage d'outils/plateformes de travail collaboratif ne suffira pas à une équipe pour qu'elle puisse prétendre faire du travail collaboratif et être efficace. Afin d'augmenter les chances de succès du travail collaboratif, les OSP doivent s'assurer d'utiliser un cadre de travail collaboratif commun. Ce cadre doit définir une méthodologie de partage, les rôles/responsabilités de chacun ainsi que les protocoles et outils de communication. Ce cadre devrait permettre de structurer le partage, faciliter les échanges et favoriser l'engagement dans I'initiative d'IO. 
L'objectif du cadre est de favoriser une communication constante entre les individus et que tout le monde soit informé de manière transparente malgré les difficultés liées aux différentes perspectives, contextes organisationnels, barrières ou connaissances et compétences. Les partenaires impliqués doivent également être consultés de manière adéquate afin de recevoir un retour d'information continue sur le développement de l'innovation, afin de comprendre l'ajustement nécessaire lié aux résultats de l'innovation et au processus de développement.

\section{Bonne pratique \#17}

\section{Surveiller le déroulement des initiatives d'IO}

Comme pour tous projets ou pour toutes initiatives organisationnelles, il est important que les OSP fassent un suivi constant et régulier de l'avancement et des dynamiques internes des initiatives d'IO. Effectivement, surveiller constamment les initiatives d'lO devrait permettre de maintenir l'engagement des partenaires et favoriser le développement d'innovations. Cette surveillance et ce suivi peuvent être réalisés en utilisant un cadre de gouvernance bien défini, mais flexible, de l'initiative d'IO, dans lequel les rôles et responsabilités de chacun des individus/partenaires impliqués sont définis. Le fait que chaque individu/partenaire sache qui doit faire quoi assure un flux efficace entre eux et facilite la collaboration. Une surveillance constante permet également d'apprendre, d'ajuster les processus selon les besoins et de suivre les nouvelles opportunités. 


\section{Bonne pratique \#18 Déployer une infrastructure informatique flexible et des données ouvertes}

Finalement, la dernière bonne pratique que les OSP devraient suivre afin d'accroître les probabilités de succès de leurs initiatives d'IO est de déployer une infrastructure informatique flexible qui peut être facilement étendue pour soutenir l'évolution de l'innovation. Les OSP devraient également promouvoir l'utilisation de l'open source et du code ouvert. De plus, si possible, les OSP devraient favoriser une approche de données ouvertes avec des référentiels de données accessibles au public pour donner la possibilité de développer l'innovation par différents partenaires. Les données ouvertes peuvent être anonymisées et/ou agrégées afin d'en assurer la confidentialité et la sécurité.

Le tableau 2 présente un résumé des 18 bonnes pratiques décrites ci-dessus. Chacune de ces bonnes pratiques représente des actions organisationnelles facilitant déploiement de capacités dynamiques de détection (De.), de capture (Ca.) et/ou de transformation (Tr.). 


\section{Tableau 2. Actions organisationnelles déployées par les OSP lors d'Initiatives d'IO*}

Cap. Dyn.

\begin{tabular}{|c|c|c|c|c|}
\hline \#BP & Actions organisationnelles & De. & Ca. & Tr. \\
\hline BP1 & Favoriser une culture d'ouverture et d'expérimentation & $\mathbf{x}$ & $\mathbf{x}$ & $\mathbf{x}$ \\
\hline BP2 & $\begin{array}{l}\text { Impliquer les partenaires à l'aide de plateformes collaboratives et } \\
\text { coordonner les échanges }\end{array}$ & $\mathbf{x}$ & $\mathbf{x}$ & $\mathbf{X}$ \\
\hline BP3 & Générer et collecter des idées à l'aide d'une plateforme numérique & $\mathbf{X}$ & & \\
\hline BP4 & Développer une présence sur les réseaux sociaux & $\mathbf{x}$ & $\mathbf{x}$ & \\
\hline BP5 & Organiser des rencontres entre les partenaires clés impliqués dans l’IO & $\mathbf{x}$ & $\mathbf{x}$ & \\
\hline BP6 & Cartographier les besoins et les capacités des utilisateurs finaux & $\mathbf{x}$ & $\mathbf{x}$ & \\
\hline BP7 & Développer des prototypes et des prétests fréquemment & & $\mathbf{x}$ & \\
\hline BP8 & Adopter une approche agile & & $\mathbf{x}$ & $\mathbf{x}$ \\
\hline BP9 & Gérer les enjeux relatifs à la gouvernance et la sécurité des données & & $\mathbf{x}$ & $\mathbf{x}$ \\
\hline BP10 & $\begin{array}{l}\text { Promouvoir une communication ouverte et transparente entre les } \\
\text { partenaires }\end{array}$ & & $\mathbf{x}$ & $\mathbf{x}$ \\
\hline BP11 & Renforcer la motivation et l'engagement des partenaires & & & $\mathbf{x}$ \\
\hline BP12 & Allouer des ressources financières suffisantes aux initiatives d'IO & & & $\mathbf{x}$ \\
\hline BP13 & Obtenir un support des hauts dirigeants & & & $\mathbf{x}$ \\
\hline BP14 & Développer un leadership fort et une vision claire et cohérente & & & $\mathbf{x}$ \\
\hline BP15 & $\begin{array}{l}\text { Identifier et sélectionner des membres d'équipe avec des } \\
\text { compétences complémentaires et une bonne attitude }\end{array}$ & & & $\mathbf{x}$ \\
\hline BP16 & Utiliser un cadre de travail collaboratif commun & & & $\mathrm{x}$ \\
\hline BP17 & Surveiller le déroulement des initiatives d'IO & & & $x$ \\
\hline BP18 & $\begin{array}{l}\text { Déployer une infrastructure informatique flexible et des données } \\
\text { ouvertes }\end{array}$ & & & $\mathbf{x}$ \\
\hline
\end{tabular}

\subsection{Analyse des bonnes pratiques liées à la DÉTECTION - Détecter les opportunités ou les menaces}

Afin de détecter de nouvelles opportunités ou menaces, les bonnes pratiques/actions organisationnelles utilisées par les OSP, lors de la mise en œuvre des initiatives d'IO, sont orientées vers le renforcement des relations entre les différents partenaires impliqués. Des occasions de rencontres, en ligne ou en personne, doivent être créées pour favoriser les interactions entre les individus et les organisations. En ligne, les OSP peuvent utiliser des plateformes d'IO dédiées afin de 
Afin de détecter de

nouvelles opportunités ou

menaces, les bonnes

pratiques/actions

organisationnelles

utilisées par les OSP, lors

de la mise en œuvre des

initiatives d'IO, sont

orientées vers le

renforcement des

relations entre les

différents partenaires

impliqués. faciliter et stimuler la génération d'idées et le partage de connaissances entre les partenaires, et/ou construire une présence en ligne sur les réseaux sociaux existants (par exemple, Twitter, Facebook).

Sur ces plateformes, les utilisateurs, qu'ils soient partenaires existants ou parties prenantes externes, peuvent soumettre des idées qui seront ensuite discutées, évaluées et/ou votées par les équipes des initiatives d'IO. De plus, les médias sociaux peuvent être utilisés pour transmettre des informations pertinentes au public ou pour collecter des idées, mais aussi pour identifier de nouvelles personnes et organisations pouvant participer ou s'impliquer dans l'effort d'innovation. Les OSP peuvent également organiser des réunions en personne, telles que des hackathons, des rallyes, des coopérathons ou des ateliers pour transmettre des informations liées aux objectifs d'innovation, afin de susciter de I'intérêt pour l'innovation et de promouvoir le développement de nouvelles idées.

Les résultats ont également montré qu'une bonne compréhension des utilisateurs potentiels (p.ex. citoyens), notamment en termes de connaissances, d'expertise et d'intérêts, est nécessaire afin d'identifier des opportunités d'innovation pouvant répondre à leurs besoins. Cela peut être fait en s'alliant à des partenaires qui travaillent directement avec les utilisateurs finaux. La génération d'idées ne se fait pas seulement par le biais d'acteurs externes, mais peut aussi provenir de l'organisation elle-même ou de l'écosystème dans lequel elle opère. Ainsi, le développement d'une culture organisationnelle qui valorise l'expérimentation et l'apprentissage expérientiel facilite ces processus et a été soulevé dans plusieurs cas.

L'ensemble des bonnes pratiques liées à la DÉTECTION d'opportunités/menaces émergentes dans son environnement peuvent être regroupées en trois catégories d'actions organisationnelles : D1 -Comprendre les parties prenantes, D2 
- Promouvoir les relations entre les partenaires internes et externes et D3 - Identifier les sources potentielles de connaissances. La figure 1 présente ces trois catégories et leurs actions sous-jacentes.

\section{Figure 1. Actions organisationnelles facilitant le déploiement de la capacité de DÉTECTION- Regroupées par catégories}

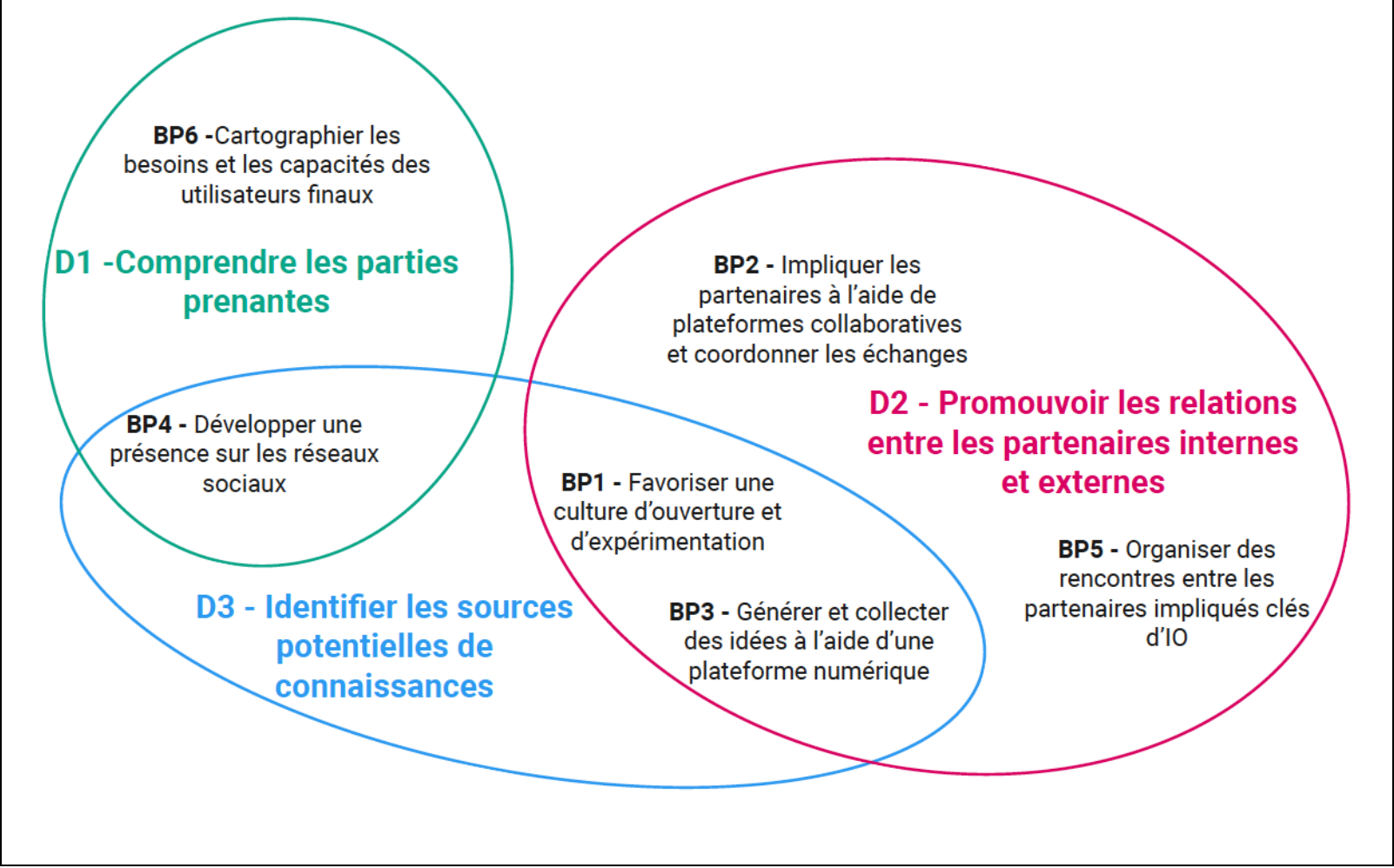

Il est essentiel que les OSP aient une bonne compréhension des utilisateurs potentiels, en termes de connaissances, de capacités et d'intérêts

\subsection{Analyse des bonnes pratiques liées à la} CAPTURE - Saisir les opportunités et éviter les menaces

Afin de capturer les opportunités d'innovation, les OSP peuvent déployer différentes bonnes pratiques / actions organisationnelles. Tout d'abord, il est essentiel que les OSP aient une bonne compréhension des utilisateurs potentiels, en termes de connaissances, de capacités et d'intérêts. Cette compréhension devrait permettre aux OSP d'identifier et de 
saisir les opportunités qui correspondent le mieux aux besoins de ces utilisateurs. Les réunions entre les partenaires impliqués dans une initiative d'IO sont également des occasions importantes de recevoir des commentaires afin de sélectionner la technologie ou le service le plus approprié à développer. Dans le même ordre d'idée, promouvoir une communication ouverte et transparente entre les partenaires devrait également permettre de faciliter la saisie d'opportunités et de développer un climat de collaboration basée sur la confiance.

En termes de processus, l'adoption de méthodes agiles et le développement de pilotes et de prétests tout au long du processus d'innovation sont deux autres pratiques/actions pouvant aider les OSP à mieux saisir les opportunités. Les pratiques agiles, ainsi que d'autres formes de développement itératif, devraient permettre aux partenaires impliqués dans des initiatives d'IO de recevoir une rétroaction continue et de s'adapter rapidement aux changements.

L'ensemble des bonnes pratiques liées à la CAPTURE d'opportunités et l'évitement des menaces dans son environnement peuvent être regroupées en trois catégories d'actions organisationnelles : C1-Comprendre les besoins des utilisateurs, C2 - Développer l'innovation et C3-Recevoir de la rétroaction en continu. La figure 2 présente ces trois catégories et leurs actions sous-jacentes. 


\section{Figure 2. Actions organisationnelles facilitant le déploiement de la capacité de CAPTURE - \\ Regroupées par catégories}

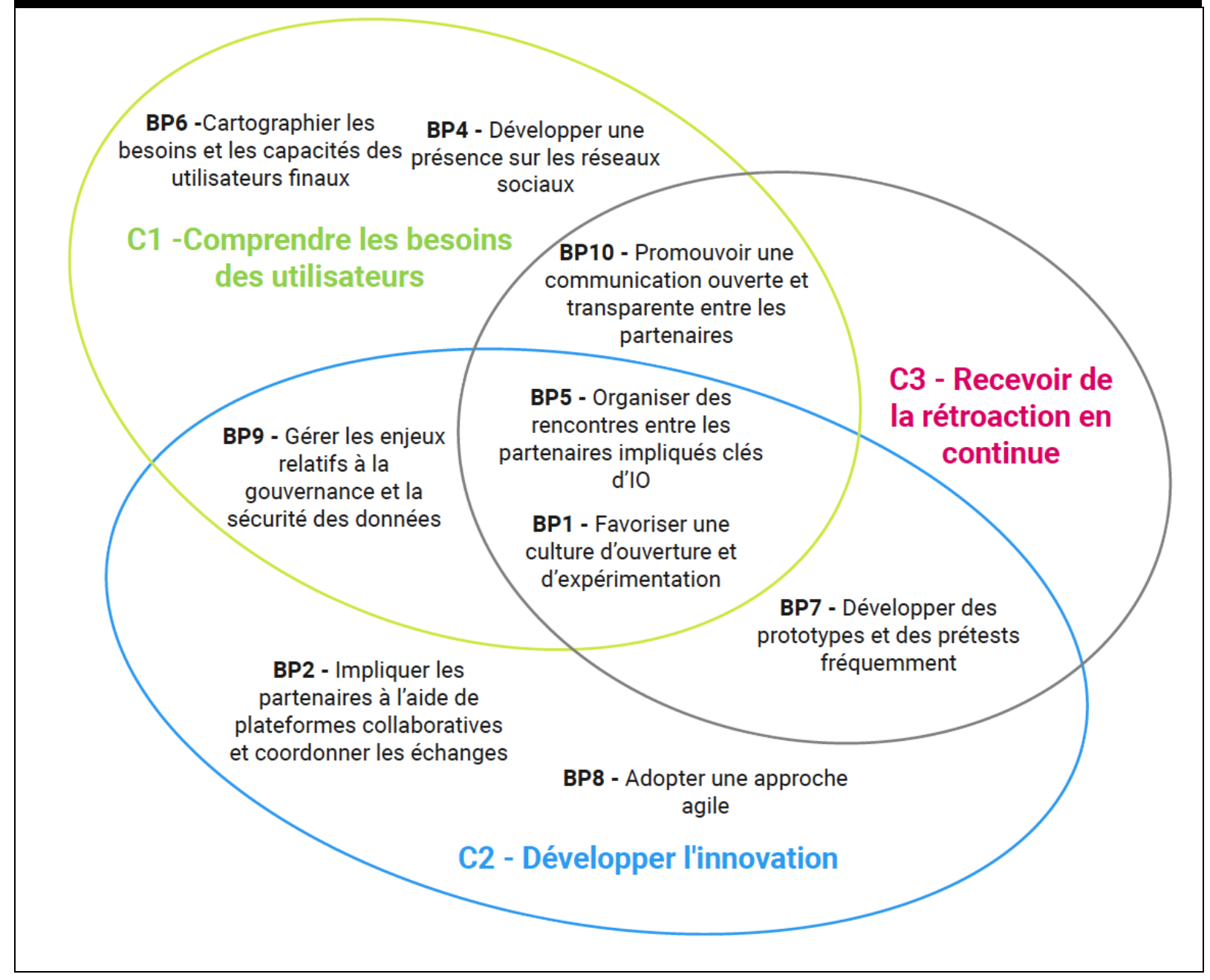

\subsection{Analyse des bonnes pratiques liées à la TRANSFORMATION - Mobiliser, combiner et reconfigurer les actifs/capacités}

Il est important de rappeler que les initiatives d'IO impliquent l'intégration des connaissances et des perspectives externes d'une pluralité de contributeurs, y compris des partenaires qui ne sont traditionnellement pas impliqués dans les processus d'innovation. Par conséquent, l'identification et la sélection de bons individus/partenaires semblent être une pratique/action essentielle au succès d'initiatives d'IO. Afin de constituer des 


\section{L'utilisation de ces plateformes numériques} devrait renforcer l'engagement des partenaires impliqués en partageant l'avancement du projet, en facilitant la communication, l'échange d'informations et la collaboration équipes d'innovation multidisciplinaires, il est nécessaire de sélectionner des individus/partenaires ayant des connaissances, des compétences diversifiées et complémentaires, tout en étant engagés dans l'initiative d'IO.

Par ailleurs, une collaboration efficace est essentielle pour mener à bien un processus d'innovation dans un contexte d'IO. Pour faciliter la collaboration, l'utilisation d'un cadre collaboratif qui définit les rôles de chaque partenaire ainsi que les protocoles et outils de communication sont importants. $\mathrm{Ce}$ type de cadre vise à faciliter la communication entre les partenaires et permet à chacun d'être informé de manière transparente malgré les difficultés liées aux différentes perspectives, contexte organisationnel, barrières ou connaissances et compétences. Une pratique/action complémentaire consiste à coordonner les efforts des partenaires à l'aide d'une plateforme collaborative. L'utilisation de ces plateformes numériques devrait renforcer l'engagement des partenaires impliqués en partageant l'avancement du projet, en facilitant la communication, l'échange d'informations et la collaboration. Enfin, le suivi des processus d'innovation et le partage de l'avancement du projet facilitent également la collaboration en s'assurant que chaque individu/partenaire sait qui doit faire quoi. Ceci est réalisé en utilisant un cadre de gouvernance bien défini, dans lequel les rôles et responsabilités de chacun sont clairement décrits. Les résultats ont également révélé que les OSP devraient être prudentes quant à l'allocation de ressources financières suffisantes tout au long des différentes phases du projet.

Du côté de la gestion, l'obtention d'un soutien de la haute direction ainsi que le développement d'un leadership fort sont également deux pratiques/actions importantes. De plus, une motivation et un engagement accrus peuvent être obtenus en renforçant la confiance dans le processus, en promouvant les avantages de l'innovation et en clarifiant les gains de chaque partenaire impliqué (p. ex. reconnaissance, récompenses monétaires, etc.) Par ailleurs, plusieurs cas ont souligné le développement d'une culture ouverte et d'innovation comme 
une pratique/action importante à déployer. Idéalement, une culture organisationnelle dans laquelle l'expérimentation et l'apprentissage organisationnel sont encouragés ainsi que la patience, le soutien et l'acceptation des risques. En effet, la communication ouverte et la transparence permettent aux individus de se sentir libres de signaler les problèmes et les échecs. Les individus devraient également être disposés à suivre et à exécuter les idées qui viennent de l'extérieur de l'organisation.

Enfin, la construction d'une infrastructure informatique flexible qui peut être facilement étendue pour soutenir l'évolution de l'innovation est un autre aspect important. Dans plusieurs cas de notre échantillon, I'utilisation de l'open source, du code ouvert et, si possible, des approches de données ouvertes avec des référentiels de données accessibles au public a été encouragée pour faciliter le développement de l'innovation par différents partenaires. Par contre, la sécurité des données est un enjeu majeur pour les OSP qui se doivent de gérer ces aspects avec attention. Le recours à des spécialistes en sécurité des données peut aider les différents partenaires à bien comprendre les standards de sécurité, s'y conformer et/ou participer à l'évolution de ces standards, tout en créant un climat de confiance.

L'ensemble des bonnes pratiques liées à la TRANSFORMATION permettant de mobiliser, combiner et reconfigurer les actifs/capacités nécessaires pour répondre rapidement aux opportunités et menaces peuvent être regroupées en quatre catégories d'actions organisationnelles: T1 - Soutenir une collaboration efficace, T2 - Développer une culture organisationnelle innovante, T3 - Obtenir l'engagement des parties prenantes et T4 - Mettre en ceuvre les ressources informatiques appropriées. La figure 3 présente ces quatre catégories et leurs actions sous-jacentes. 


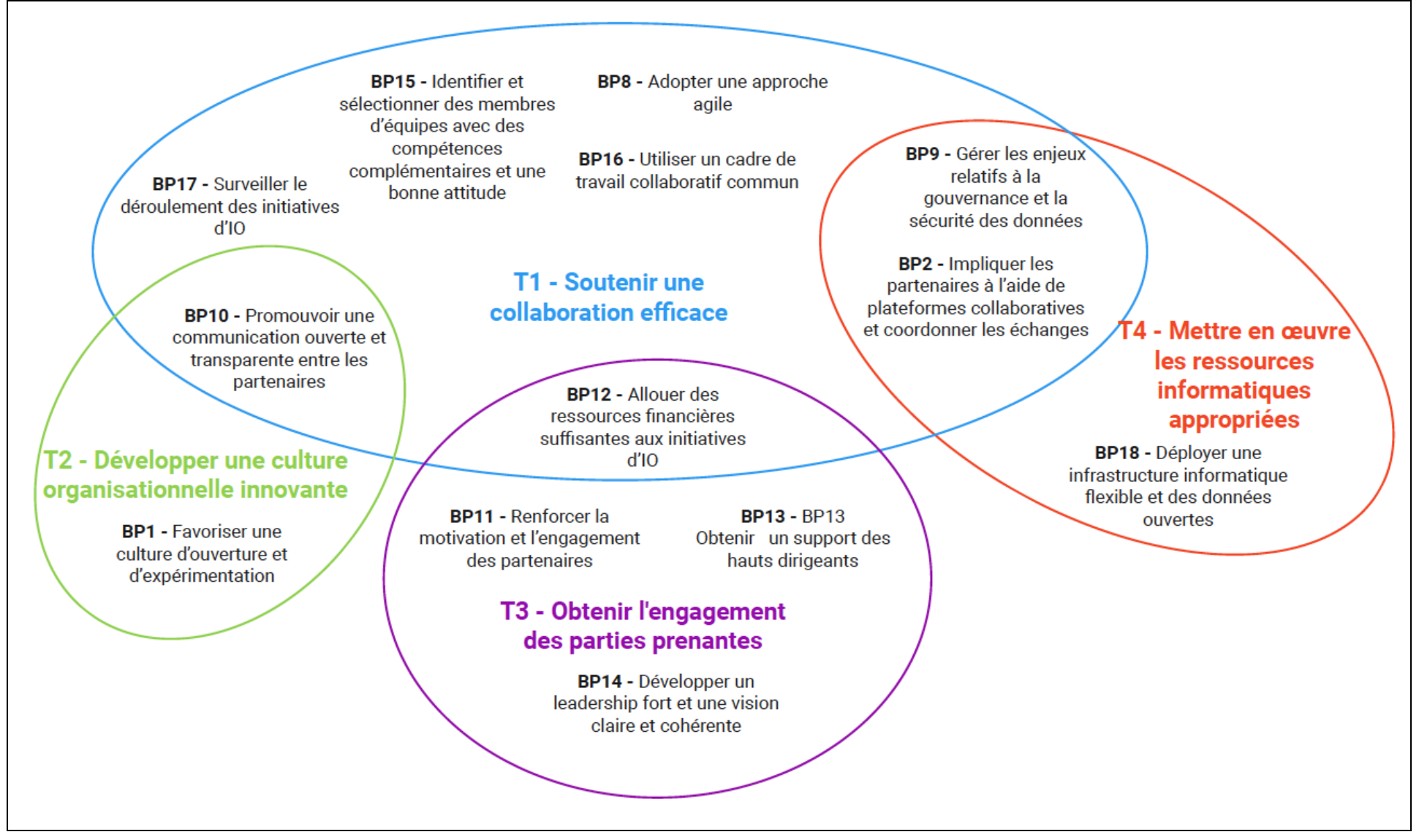

Nos résultats suggèrent que les TI ont un rôle omniprésent, car elles prennent en charge ou supportent la plupart des actions déployées par l'organisation

\subsection{Bonnes pratiques d'innovation ouverte et rôle des technologies de l'information}

À travers une analyse de 253 études de cas « exemplaire " d'IO dans des OSP, nous avons identifié 18 bonnes pratiques/actions organisationnelles à travers lesquelles les capacités dynamiques de détection, de capture et de transformation sont mises en œuvre pour réaliser des initiatives d'IO. Ces pratiques/actions ont été déployées pour faire face aux défis et aux opportunités sous-jacents aux initiatives d'IO.

Ainsi, lorsque nous examinons les pratiques/actions, nos résultats suggèrent que les technologies de l'information (TI) ont un rôle omniprésent, car elles prennent en charge ou supportent la plupart des pratiques/actions déployées par I'organisation. En particulier, nos résultats suggèrent que les TI 
ont été utilisées pour, entre autre : 1) soutenir le traitement et le partage des informations; 2) faciliter l'acquisition, l'interprétation, l'assimilation, la transformation et l'utilisation des connaissances ; 3 ) aider à gérer les ressources et les tâches ; 4) synchroniser les activités ; 5) améliorer la communication ; 6) favoriser le travail collaboratif et 7) permettre des flux d'informations et de connaissances en temps réel. Toutefois, afin d'utiliser efficacement ces technologies, un aspect que les OSP ne doivent pas négliger est de disposer des compétences technologiques appropriées en matière d'IO ou de la capacité des unités de travail d'IO à utiliser efficacement les fonctionnalités $\mathrm{TI}$ pour soutenir les activités $\mathrm{d}^{\prime} 1 \mathrm{O}^{58}$.

58 (Pavlou and El Sawy 2006; Pavlou and El Sawy 2010) 


\section{4 Écosystème d'innovation : Deux études de cas : PULSAR et CISAM}

\section{1 Étude de cas : PULSAR et CISAM}

Afin de mieux comprendre comment les bonnes pratiques identifiées peuvent aider les OSP à répondre aux défis liés à I'IO, tout en favorisant la création de valeur lors du cycle d'innovation, deux études de mise en place d'écosystèmes d'innovation, impliquant des OSP, ont été réalisées : PULSAR et CISAM. Ces deux études de cas sont décrites et analysées dans les sections qui suivent. L'annexe 3 présente les détails de l'approche méthodologique suivie.

À la fois virtuel et réel, l'espace PULSAR rassemble des partenaires et des acteurs de tout horizon dont l'objectif est d'améliorer de façon significative et durable la santé et le bien-être de la population québécoise

\subsubsection{PULSAR - Description, partenaires et santé durable} Une première étude de cas a été réalisée au Québec chez PULSAR $^{59}$ qui est un espace collaboratif de recherche et d'innovation en santé durable mise en œuvre par l'Université Laval (UL) et Alliance santé Québec (AsQ). À la fois virtuel et réel, l'espace PULSAR rassemble des partenaires et des acteurs de tout horizon dont l'objectif est d'améliorer de façon significative et durable la santé et le bien-être de la population québécoise. PULSAR est à la fois, une plateforme, une communauté, un réseau de partenaires et d'acteurs, plusieurs projets et une banque de données sur la santé durable. Qu'estce que la santé durable ? Voici la vision de santé durable qui dicte PULSAR et ses partenaires :

La santé durable, c'est une vision contemporaine de la santé qui va beaucoup plus loin que la conception classique. Elle englobe toutes les facettes de la santé et du bien-être de chaque individu, et prend en considération tout ce qui peut les affecter, positivement ou négativement. Pour que la santé soit réellement durable, il importe d'agir aujourd'hui tout en pensant à demain. Cela signifie de donner à tous un accès

${ }^{59}$ https://pulsar.ca/accueil 
équitable à des milieux et à des ressources qui favorisent le développement et le maintien de saines habitudes de vie. Cela veut dire aussi d'avoir une vision à long terme pour que les générations à venir puissent, elles aussi, vivre en santé. Et puisque la santé des êtres humains est étroitement liée à celle de la planète, la santé durable réfère également au maintien ou à l'amélioration de l'état de l'environnement naturel, dans une perspective de développement durable. Donc pour nous, la santé durable c'est :

\section{"Un esprit sain dans un corps sain, dans un milieu de vie et un environnement sains, sur une planète en santé. » 60}

\section{PULSAR est une plateforme qui met en relation divers partenaires et acteurs afin de favoriser le partage et la valorisation de données et de connaissances en lien avec la santé durable.}

PULSAR est une initiative unique au Québec, et un des rares projets similaires dans le monde ayant dépassé le stade de la conceptualisation, qui offre des solutions technologiques et collaboratives dédiées à la recherche en santé durable. Plus concrètement, PULSAR est une plateforme qui met en relation divers partenaires et acteurs afin de favoriser le partage et la valorisation de données et de connaissances en lien avec la santé durable. Ce partage et cette valorisation s'effectuent par l'entremise de projets interdisciplinaires qui visent à améliorer la santé et le bien-être de la population. Une des spécificités de PULSAR est le rôle clé joué par les citoyens qui peuvent s'impliquer et influencer positivement la recherche et les interventions en matière de santé. La figure 4 présente les alliances stratégiques et les principaux partenaires de PULSAR.

\footnotetext{
60 La définition de santé durable présentée ici a été extraite, mot pour mot, de celle présentée et utilisée par PULSAR : https://pulsar.ca/la-sante-durable?lang=fr
} 


\section{Figure 4. Alliances stratégies et partenariats}

ALLIANCES

STRATÉGIQUES

ET PARTENARIATS

PULSAR rassemble un vaste réseau de partenaires en santé, en recherche et en innovation

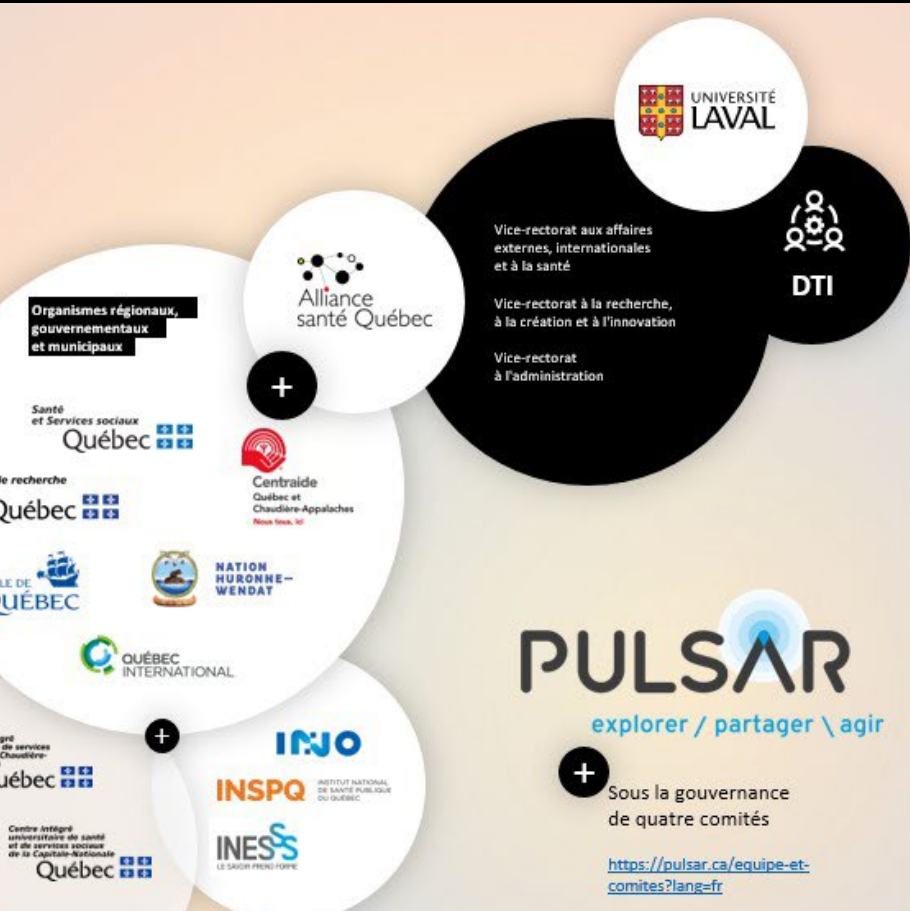

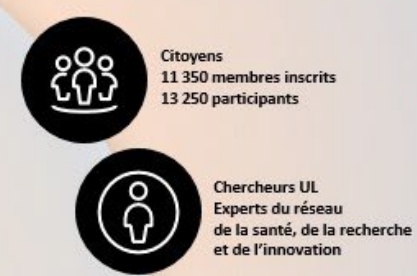

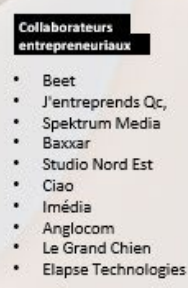

PULSAR est une initiative dont la genèse découle de l'idée de son partenaire fondateur, l'AsQ, de rassembler, dès 2013-14, un vaste réseau de partenaires régionaux, gouvernementaux, municipaux et entrepreneuriaux autour de la recherche et de l'innovation en santé durable. Ce n'est qu'en 2017 que PULSAR a été lancé et mis en œuvre avec la collaboration de 3 vicerectorats de I'UL autour de la démarche en santé durable. L'objectif à la base de PULSAR était de fédérer des acteurs clés en santé durable afin d'accélérer la recherche et l'innovation. Ainsi, la santé est abordée sous l'angle de la durabilité et préconise une approche globale et préventive, plutôt qu'une approche réactive et curative ou de médecine personnalisée. 
PULSAR adopte une approche collaborative qui cible toutes les partenaires de la communauté de recherche en santé durable

\subsubsection{PULSAR - Mission, cadre d'évaluation de la santé durable et services technologiques}

PULSAR est le fruit de nombreux échanges et collaborations avec plusieurs partenaires de I'UL, du réseau de la santé et d'autres organisations régionales. Aujourd'hui, la mission de PULSAR est d'accélérer la recherche en santé durable et d'améliorer la santé et le bien-être de la population québécoise. Pour réaliser sa mission, PULSAR ${ }^{61}$ compte sur une équipe de 17 personnes possédant des expériences et des expertises variées et complémentaires. PULSAR compte également sur l'expertise et les conseils de quatre comités soit un comité directeur, un comité scientifique, un comité de partenaire et un comité des représentants du public.

PULSAR adopte une approche collaborative qui cible tous les partenaires de la communauté de recherche en santé durable : chercheurs, citoyens, intervenants en santé et décideurs. En focalisant et en explorant les multiples déterminants de la santé physique, mentale et sociale, PULSAR et ses partenaires espèrent voir émerger une nouvelle façon de mener la recherche en santé. Ainsi, PULSAR souhaite voir la population devenir un acteur de changement au niveau de la santé durable et influence positivement la recherche et les interventions en matière de santé durable. PULSAR est toujours en développement et l'innovation ouverte fait partie de I'ADN de PULSAR.

Au niveau des chercheurs, PULSAR souhaite aider et accompagner ces derniers dans leurs projets en santé durable afin de les faire évoluer plus rapidement, de manière collaborative, avec un partage et un accès aux données plus important ainsi qu'avec des retombées concrètes. Au niveau des intervenants en santé, PULSAR souhaite pouvoir sensibiliser et orienter ces derniers au niveau du concept de santé durable et des implications sur leurs interventions auprès des personnes accompagnées. Au niveau des citoyens, l'un des aspects clés de la mission de PULSAR est de les sensibiliser et

\footnotetext{
61 https://pulsar.ca/equipe-et-comites?lang=fr
} 


\section{L'une des retombées} importantes du développement de ce cadre et du changement de culture par rapport à la santé durable est que tous les projets maintenant réalisés avec PULSAR tentent d'avoir un impact concret sur la santé de la population les impliquer au niveau de la prise en charge, de manière plus proactive et autonome, de leur santé. Finalement, au niveau des décideurs, PULSAR souhaite les guider et les orienter dans le développement de services et politiques qui répondent davantage aux besoins des citoyens et qui optimisent les dépenses en santé.

L'un des premiers chantiers majeurs sur lequel s'est penché PULSAR, qui découle d'une recommandation provenant d'une étude effectuée par un doctorant spécialisé en santé durable fut l'élaboration d'un cadre d'évaluation de la santé durable. Ce cadre, qui a mobilisé plus de 200 chercheurs de I'UL et leurs collaborateurs, a permis de/d': 1) clarifier le concept de santé durable, 2) partager des expertises et des connaissances, 3) initier de nouvelles collaborations, et 4) élargir la portée et la collecte de données de certains projets. L'une des retombées importantes du développement de ce cadre et du changement de culture par rapport à la santé durable est que tous les projets maintenant réalisés avec PULSAR tentent d'avoir un impact concret sur la santé de la population. À titre d'exemple, le projet NutriQuébec permet aux participants de recevoir un bilan nutritionnel personnalisé en ligne et le projet PERSEPECTIVE permet aux participantes de consulter en ligne leur risque de développer un cancer du sein, le tout selon une approche préventive.

Par ailleurs, afin de sensibiliser, d'accompagner et de guider ses partenaires, PULSAR utilise le numérique et l'intelligence collective (engagement et mobilisation citoyenne) pour trouver des solutions concrètes au bénéfice de la santé et du bien-être des collectivités. Depuis les débuts du projet, l'offre de services de PULSAR se distingue par l'adoption des principes FAIR de gestion et $d$ 'intendance des données (Facilement trouvables, Accessibles, Interopérables, Réutilisables) ${ }^{62}$. PULSAR propose une offre de service technologique favorisant :

\footnotetext{
62 Ces principes, mis de l'avant par le bureau de la Conseillère scientifique en chef du premier ministre du Canada, et plus récemment ( 15 mars 2021) par les 3 organismes fédéraux de financement de la recherche (IRSC, CRSNG, CRSH), visent ultimement à
} 
La mise en commun des connaissances en santé durable et l'esprit de partage sont au coeur même de la mission de PULSAR
- Le partage scientifique et la réutilisation des données

- Le travail en interdisciplinarité - intersectorialité

- Les retombées concrètes pour le citoyen

- Une démarche éthique

- L'engagement du citoyen

PULSAR réalise des projets collaboratifs et interdisciplinaires regroupant des chercheurs de disciplines très variées, ainsi que des décideurs, des citoyens et/ou des intervenants en santé. La mise en commun des connaissances en santé durable et l'esprit de partage sont au cœur même de la mission de PULSAR. L'un des projets phare de PULSAR, la Banque de données en santé durable (BDSD) initiée, développée et maintenue par PULSAR et ses partenaires, est une manifestation claire et concrète de cette nouvelle culture. L'idée de la BDSD est de favoriser la valorisation des données qui y sont déposées et qui pourront être réutilisées dans le futur dans le cadre d'autres projets, par exemple à des fins de croisements et d'analyse. La BDSD repose sur les infrastructures du Centre de Valorisation des Données $(C V D)^{63}$ de l'UL qui permet de stocker et d'analyser des données sensibles, avec des outils et des techniques de pointe, en toute sécurité. Le CVD est exploité par une équipe d'experts en gestion et en valorisation des données VALERIA ${ }^{64}$. La figure 5 présente la communauté de recherche gravitant autour de PULSAR ainsi que les principaux services offerts.

ce que les données puissent être accédées, interprétées et réutilisées par des agents humains, mais surtout par des " agents machine ».

63 https://www.ulaval.ca/notre-universite/salle-de-presse/communiques-depresse/communiques-2019/le-centre-de-valorisation-des-donnees-pret-aaccueillir-des-milliards-de-donnees

${ }^{64}$ https://valeria.science/accueil 


\section{Figure 5. PULSAR - Communauté et services}

Communauté de recherche en santé durable

Met en relation les acteur qui partagent une vision durable de la santé

Accélérateur de projets visant à contribuer concrètement

à la santé durable

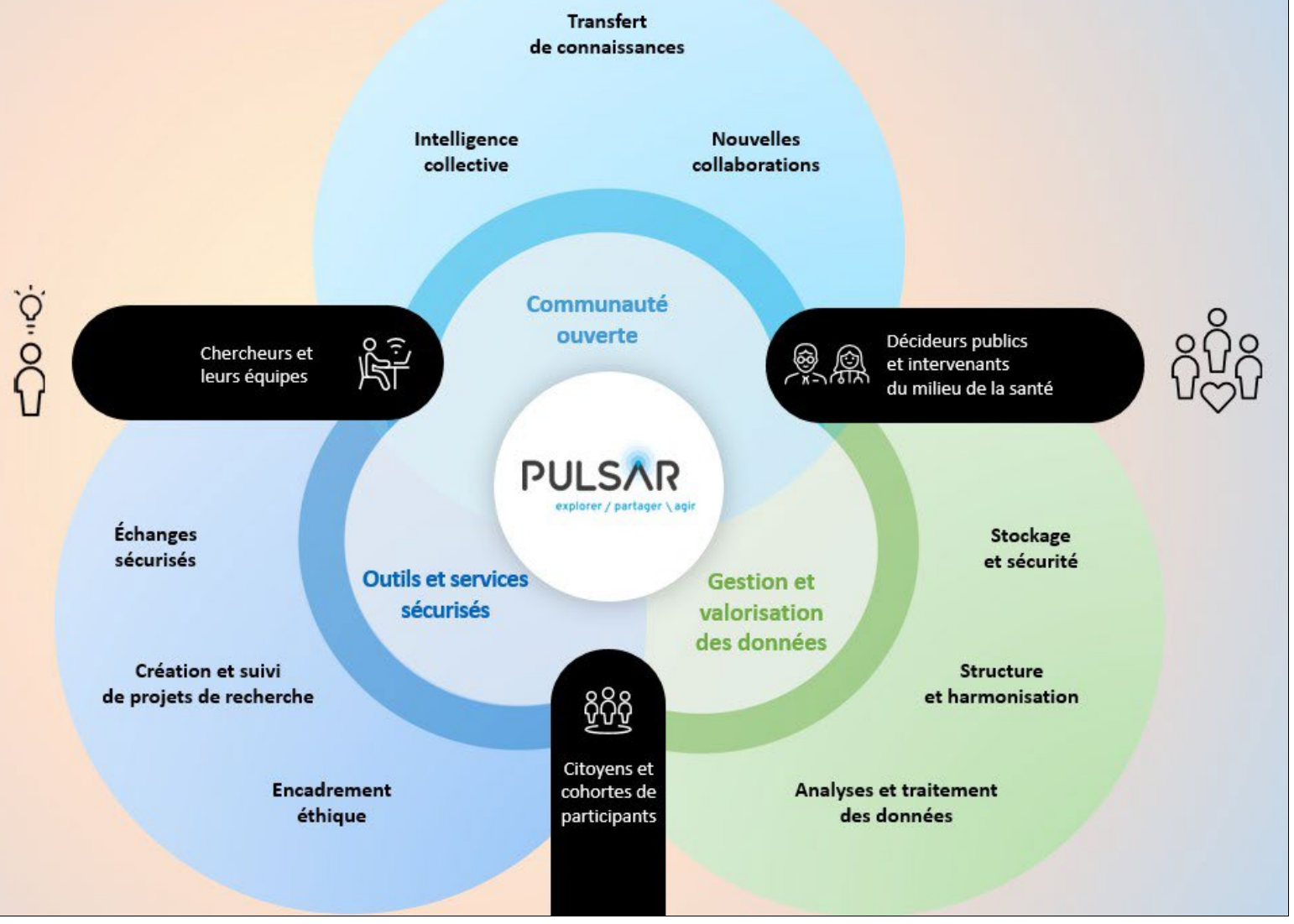

En plus du cadre d'évaluation de la santé durable et de ses services numériques, PULSAR peut compter et joindre plusieurs milliers de citoyens inscrits. Cette visibilité permet à PUSLAR de diffuser de l'information en lien avec des dizaines d'évènements liés à la santé durable et publier une vingtaine d'articles accessibles sur son site Web ainsi que sur les médias sociaux. Cette diffusion permet également à PULSAR de faire de la vulgarisation scientifique, de partager les accomplissements ainsi que de rassembler la communauté d'organisations et d'individus intéressés et interpellés par les enjeux de santé durable. De plus, PULSAR utilise des outils de communication numérique afin d'ouvrir ses portes pour présenter et attirer d'autres projets de recherche, valoriser les données et stimuler la recherche. 
La philosophie PULSAR se résume en quatre points : 1) contribuer à la santé durable, 2) impliquer les citoyens et citoyennes, 3)

être intersectoriel et interdisciplinaire, et 4) être éthique et sécuritaire.

Ces quatre éléments guident l'ensemble des actions des partenaires de l'écosystème PULSAR ainsi que le déroulement des différentes initiatives liées à PULSAR. Ces éléments jouent le rôle de " contrat moral » entre les partenaires de l'écosystème.

\subsubsection{PULSAR - Pratiques déployées pour favoriser I'innovation ouverte}

Vision, santé durable et philosophie - Lorsque PULSAR a été lancé en 2017, le concept de santé durable était peu connu et aucune définition/compréhension commune ne faisait l'unanimité entre les spécialistes. La convergence des visions a également été difficile au début, car il y avait beaucoup de parties prenantes impliquées, des confrontations au niveau des intérêts et des objectifs ainsi que des besoins non clairement identifiés. De plus, peu d'intervenants, aussi bien dans le domaine de la recherche que celui des professionnels, ne semblaient croire au projet. Afin de surmonter ces défis, les deux premières actions de la responsable initiale de PULSAR ont été de/d' 1) établir un mandat clair, simple et qui tenait en une seule phrase: Mettre en place une plateforme collaborative de recherche et d'intervention en santé durable, et 2) mobiliser les partenaires et acteurs des milieux académiques et professionnels afin d'établir une définition commune et opérationnelle du concept de santé durable. Les extrants de ces deux premières actions allaient permettre de délimiter les « frontières » du projet et de l'écosystème PULSAR en plus de guider les actions subséquentes comme, par exemple, la mise sur pied de chantiers sur la mobilisation et participation citoyenne, la mobilisation scientifique ou encore la vitrine et l'accélération des innovations.

En plus d'une vision claire et d'une définition commune, une autre action a joué un rôle essentiel au niveau de l'approche et du mode de fonctionnement de PULSAR depuis ses débuts: l'établissement de la "philosophie " PULSAR. Cette philosophie se résume en quatre points: 1) contribuer à la santé durable, 2) impliquer les citoyens et citoyennes, 3) être intersectoriel et interdisciplinaire, et 4) être éthique et sécuritaire. Ces quatre éléments guident l'ensemble des actions des partenaires de l'écosystème PULSAR ainsi que le déroulement des différentes initiatives liées à PULSAR. Ces éléments jouent le rôle de "contrat moral " entre les partenaires de l'écosystème. 


\section{La détermination,}

l'engagement, le

dévouement et l'attitude

positive des membres de

l'équipe expliquent en

bonne partie le succès de

PULSAR
Équipe, attitude et comités - Afin de déployer ses différents projets et d'obtenir éventuellement l'implication d'autres partenaires et d'acteurs du milieu, la responsable de PULSAR a formé une toute petite équipe de travail en embauchant une chargée de projet et une analyste d'affaires. Elle a souligné que ce sont particulièrement la détermination, l'engagement, le dévouement et l'attitude positive de ces deux personnes qui expliquent en bonne partie le succès de PULSAR. Cette petite équipe a eu, au départ, "à tenir le projet à bout de bras ". En plus de sélectionner et de s'entourer d'une équipe dont les membres possédaient des compétences complémentaires et une attitude constructive, un comité directeur a été formé à l'automne 2017, ce qui a permis à PULSAR d'aller chercher du support à trois niveaux: 1) Politique, par l'entremise de $\mathrm{Dr}$ Rénald Bergeron, vice-recteur aux affaires externes, internationales et à la santé de I'UL; 2) Recherche, par l'entremise de Mme Eugénie Brouillet, vice-rectrice à la recherche, à la création et à l'innovation de l'UL, et 3) Financier, par l'entremise de M. André Darveau, vice-recteur de I'UL. Ce comité directeur a été complété par deux autres acteurs clés : M. Dave Fiset, directeur de la direction des technologies de I'information à l'UL et M. René Lacroix, vice-recteur adjoint à l'administration.

Il est important de souligner que PULSAR est parti d'un projet et non d'une grosse structure. Lorsque PULSAR a été lancé, il n'y avait pas de structure existante. L'équipe de PULSAR a eu à la créer et la structure a évolué au fur et à mesure avec les projets et leurs besoins. Chez PULSAR, il n'y a toujours pas de structure finale. Elle est en constante évolution et en adaptation.

Parties prenantes et clients - Lorsque la responsable initiale de PULSAR a obtenu le mandat de lancer et déployer l'initiative PULSAR, une des premières choses qui a été effectuée fut d'identifier, de cartographier et d'analyser l'ensemble des parties prenantes de l'écosystème PULSAR. Ce faisant, l'équipe PULSAR a pu répertorier les expertises, les capacités et les besoins de l'ensemble des parties prenantes. Les parties 


\section{L'analyse de parties} prenantes a permis de tracer la carte " politique » du projet ainsi que d'identifier les enjeux actuels et à venir. prenantes ont été regroupées dans quatre grands groupes: chercheurs, décideurs, intervenants du milieu de la santé et les citoyens. Cet exercice a également permis de tracer la carte " politique " du projet ainsi que d'identifier les enjeux actuels et à venir. Suite à cet exercice, l'équipe a pu établir des principes de travail collaboratif ainsi que des cibles à atteindre. Puisqu'il y avait plusieurs groupes de parties prenantes avec des demandes/exigences divergentes, l'équipe de PULSAR a dû prioriser. Il a été décidé de débuter avec le volet recherche et les chercheurs. Cette décision découle du fait que, tout comme PULSAR, les chercheurs se trouvaient dans le mur de UL, donc plus facile d'accès. De plus, ces derniers avaient déjà de l'expertise et de l'expérience en lien avec les exigences des comités éthiques. La recherche et les données sont également le cœur de PULSAR. Finalement, les premiers utilisateurs/bénéficiaires des services de PULSAR étaient les chercheurs. Par la suite, les besoins et exigences des autres groupes de parties prenantes furent intégrés.

Par ailleurs, cinq thèmes clés essentiels à la réussite de PULSAR ont également été identifiés: 1) Éthique, 2) Gouvernance, 3) Santé durable, 4) Implication citoyenne et 5) Sécurité et TI. Un chercheur-parrain a été mandaté pour mener les ateliers et les tables de concertations en lien avec chacun de ces thèmes. L'équipe de PULSAR fut en support lors de ces ateliers afin de structurer et documenter les échanges et discussions.

Collaboration et momentum - Lors du lancement du projet, il $y$ avait une certaine instabilité et certains enjeux à l'As $Q$, qui est l'un des principaux initiateurs et partenaires de PULSAR. Or, malgré cette situation qui aurait pu retarder l'émergence de PULSAR, l'équipe de PULSAR a décidé de travailler malgré tout avec la communauté en créant des comités citoyens et des comités d'affaires. Ces comités qui initialement auraient dû être créés et chapeautés par I'AsQ ont finalement été pris (temporairement sur approbation des directions concernées) en charge par l'équipe de PULSAR afin de ne pas retarder le projet. Ainsi, en travaillant avec des partenaires, sur des besoins réels et concrets, l'équipe de PULSAR a pu maintenir le 


\section{PULSAR a toujours}

\section{accueilli les partenaires}

qui souhaitaient s'investir

dans PULSAR et a

maintenu une atmosphère

de collégialité, d'ouverture

et de transparence avec

tous les partenaires. momentum suite au lancement initial du projet. D'ailleurs, I'un des principes clés de gestion chez Pulsar a toujours été l'hyper collaboration qui se résume ainsi: $\mathrm{Si}$, en tant qu'individu ou organisation, tu ne collabores pas à l'objectif commun de PULSAR, si tu ne veux pas t'impliquer, pas de soucis. PULSAR continue à avancer uniquement avec ceux et celles qui souhaitent s'impliquer et travailler pour l'objectif commun. Tu te mets sur le côté et tu embarqueras lorsque tu souhaiteras $t^{\prime}$ investir et $t^{\prime}$ impliquer. L'équipe PULSAR travaille donc toujours avec des experts qui sont intéressés et intéressants.

Au début, certains chercheurs regardaient le travail de PULSAR de haut, sans trop vouloir s'impliquer. Lorsqu'ils ont vu et compris les bénéfices, plusieurs se sont impliqués dans l'aventure. La responsable du projet a toujours accueilli les partenaires qui souhaitaient s'investir dans PULSAR et elle a surtout maintenu une atmosphère de collégialité, d'ouverture et de transparence avec tous les partenaires. D'ailleurs, la responsable du projet a souligné le fait que, dans un écosystème comme celui de PULSAR, mieux vaut focaliser son énergie et son attention sur les partenaires "positifs " qui servent de locomotives, qui déteignent sur les autres et créent un effet d'entraînement ainsi que sur les "neutres" qui peuvent éventuellement embarquer, plutôt que perdre son temps et son énergie avec les partenaires "négatifs " qui ralentissent l'initiative.

Positionnement - Deux options de positionnement possibles s'offraient aux responsables de PULSAR lors de son lancement : soit en faire un projet purement " technologique " ou soit en faire un projet d'innovation. La petite équipe de PULSAR a évalué les deux options en analysant les cadres de gouvernance nécessaires à mettre en place ainsi que les avantages et inconvénients de deux options. Il a été choisi d'en faire un projet d'innovation plutôt qu'un projet uniquement technologique qui aurait été restreignant. Afin d'augmenter les probabilités de succès, la responsable du projet et son équipe sont allées chercher, auprès du comité directeur, le support politique, financier et en recherche pour que ça fonctionne. Par 
Aucune décision majeure ou stratégique, en lien avec l'objectif, ne s'est prise sans avoir consulté, ni avoir l'appui des différents comités de partenaires, soit les comités scientifiques, d'affaires et de citoyens ailleurs, une autre pratique clé qui a été mise en place par l'équipe de PULSAR a été de valider rapidement les idées et propositions importantes avec tous les partenaires de l'écosystème. Aucune décision majeure ou stratégique, en lien avec l'objectif, ne s'est prise sans avoir consulté ni avoir l'appui des différents comités de partenaires, soit les comités scientifiques, d'affaires et de citoyens. Cette pratique a permis de maintenir l'implication des partenaires et de profiter de leurs expertises. Par exemple, de grandes "messes" regroupant parfois près de 150 partenaires de différents milieux ont été organisées afin de faire des séances d'idéation.

Agilité et co-construction - Dès le début du projet PULSAR, l'équipe de PULSAR a présenté au vice-recteur de I'UL, les principes d'innovation et d'agilité qui allaient guider le développement de l'écosystème PULSAR c.-à-d. faire des sprints d'un ou deux mois, faire de petites livraisons fréquentes, collecter les rétroactions et se réajuster. Comme l'a mentionné la responsable de PULSAR, il n'y avait pas d'anticipation, pas de plan exhaustif, pas d'analyse très détaillée, mais tout était structuré, un peu comme dans la méthode Scrum. L'équipe PULSAR avançait par itérations en identifiant les besoins et en travaillant sur les priorités. Comme l'a mentionné une analyste d'affaires de PULSAR, l'équipe a commencé par livrer ce qui donnait le "plus de valeur ", ce qui était prioritaire en fonction des projets des chercheurs proposés. PULSAR opère avec une approche par petits pas en partant de son noyau et en construisant autour de sa vision. L'équipe de PULSAR dispose également d'un état d'esprit qui accepte que tout ce qui sera fait ne sera pas parfait, car il est impossible de tout prévoir, ce qui est contraire à la philosophie scientifique qui s'attarde sur les détails qui deviennent souvent des freins. De plus, pour qu'une initiative comme PULSAR fonctionne dans un contexte universitaire très bureaucratique, deux pratiques ont été mises en avant par l'équipe PULSAR : 1) Avoir un " mindset » de service à la clientèle et 2) Faire de la « vraie » co-construction. 
PULSAR opère avec une approche par petits pas en partant de son noyau et en construisant autour de sa vision

\section{La clé est de savoir} comment choisir les opportunités à prioriser, c'est-à-dire celles qui sont alignées avec les objectifs et avec la vision de PULSAR
Tout d'abord avoir un mindset client signifie : bien identifier le ou les clients existants et potentiels de PULSAR, tenter de se mettre dans la peau de ses clients et ramener constamment ces derniers au cœur de toutes les discussions. En ce qui concerne la co-construction, il s'agit de faire des séances de brainstorming, à distance ou en présentiel, avec tous les partenaires afin d'avoir la plus grande variété d'idées et d'aller chercher l'adhésion. La co-construction peut créer de I'inconfort, car il arrive que certains participants souhaitent revenir à des façons de travailler qu'ils/elles connaissent. Il est important d'être bien préparé et d'avoir des structures pour animer des séances de co-construction. Par exemple, pour trouver le nom PULSAR, une quarantaine de personnes ont participé à un atelier de brainstorming où cinq noms ont été retenus et, par la suite, un vote a été tenu auprès de tous les partenaires. Ce principe de co-construction, qui est au cœur même du fonctionnement de l'écosystème PULSAR, a également été utilisé pour développer, en toute transparence, la vision/mission de PULSAR avec les partenaires. Pour se faire, divers ateliers structurés ont été organisés. Ces ateliers ont été encadrés avec méthode, les objectifs définis sous forme de questions claires et structurées préparées à l'avance, et envoyées à l'avance également aux participants.

Opportunités et apprentissages - Une autre caractéristique clé du mode de fonctionnement de PULSAR est son approche "startup", c'est-à-dire que PULSAR est ouverte et à la recherche d'opportunités. Comme l'a mentionné une analyste d'affaires, PULSAR baigne dans une mer d'opportunités et avance par opportunité. La clé est de savoir comment choisir les opportunités à prioriser, c'est-à-dire celles qui sont alignées avec les objectifs avec la vision de PULSAR. Par exemple, des projets se sont présentés avec des besoins pour lesquels certaines fonctionnalités n'existaient pas sur la plateforme technologique de PULSAR. L'évaluation et la sélection de ces opportunités se sont faites en fonction de l'alignement de ces projets avec la vision de PULSAR, c'est-à-dire l'existence d'un lien avec la santé durable, ainsi qu'au niveau de la bonification 
La philosophie de PULSAR est que l'apprentissage organisationnel à chaque projet est transférable vers le prochain projet. possible de la plateforme, c.-à-d. est-ce que ces nouvelles fonctionnalités seront utiles à d'autres projets dans l'écosystème PULSAR. Lorsque c'était le cas, les nouveaux projets devaient par la suite être présentés et validés avec les autres projets et les partenaires de PULSAR avant d'être réalisés.

Ce mode de fonctionnement par opportunité a permis à PULSAR de se donner de la visibilité, d'apprendre, d'élargir son champ d'action, de développer de nouvelles relations d'affaires en plus de "recruter » de nouveaux chercheurs. II est arrivé à l'équipe de PULSAR de présenter des projets qui n'ont pas été retenus par les instances décisionnelles. Par contre, ces projets ont tout de même permis de tester des idées, d'avoir de la rétroaction, de mieux cibler les besoins des clients, de mieux comprendre comment répondre à ces besoins et de faire émerger d'autres idées de projets qui eux aussi ont réussi. La philosophie de PULSAR est que l'apprentissage organisationnel à chaque projet est transférable vers le prochain projet. De plus, il faut rappeler que l'un des objectifs de PULSAR est de rapprocher les chercheurs, les décideurs, les intervenants en santé et les citoyens afin que la connaissance développée soit utilisée par les décideurs pour en faire bénéficier les citoyens. PULSAR se veut donc un écosystème apprenant.

Données au cœur de PULSAR - L'un des principaux objectifs de PULSAR est de permettre la collecte et le partage de données liées à la santé durable. Ces données proviennent de projets de recherche rigoureusement évalués et approuvés par le comité scientifique de PULSAR. Afin de respecter les exigences éthiques, légales et technologiques entourant le dépôt, le partage et l'utilisation de ces données, plusieurs protocoles d'approbation ont été développés et mis en place pour assurer l'intégrité, la sécurité et la qualité des données. Ces protocoles, qui encadrent aussi bien les données structurées que les données non structurées des projets de recherche, sont primordiaux afin de développer la confiance des partenaires de l'écosystème PULSAR ainsi que l'ensemble de la population. Beaucoup d'efforts et d'énergie ont été investis par l'équipe de 
PULSAR pour s'assurer d'avoir un cadre de gouvernance des données clair, transparent et sécuritaire. Le comité responsable des aspects légaux et de sécurité chez PULSAR a réussi, avec beaucoup de patience, à faire changer les comités éthiques institutionnels sur deux aspects : 1 ) la pérennité des données (plutôt que d'avoir des durées limitées à 5 ans par exemple) et sur l'idée que 2) toutes les données peuvent servir ou contribuer à la santé durable. D'ailleurs, la BDSD opère avec une approche de consentement qui lui est propre, ce qui représente une innovation majeure en soi.

En plus du cadre de gouvernance des données, PULSAR prévoit à terme créer des " data lake » de données non structurées qui pourront être utilisées plus tard, car on ne sait pas encore tout ce qu'on peut tirer de ces données. Faire accepter l'idée que PULSAR souhaitait conserver les données même si personne ne savait encore ce que PULSAR et ses partenaires allaient en faire a exigé beaucoup d'énergie pour faire comprendre et accepter le tout par les comités éthiques. De nouveaux outils d'analyse pourraient émerger, de nouveaux besoins pourraient émerger et ces " data lake " pourraient être utiles. Toutes ces données sont disponibles aux partenaires qui suivent les protocoles d'approbation de PULSAR.

Par ailleurs, en ce qui a trait aux exigences de sécurité des données, et en particulier aux données humaines, des niveaux de sécurité et de sensibilisation très élevés ont été établis. Ce travail a été effectué en collaboration entre l'équipe de PULSAR et des conseillers en sécurité qui ont établi des exigences de sécurité et des avis de sécurité et d'audit pour améliorer continuellement les niveaux de sécurité. Comme l'a mentionné une analyse d'affaires de PULSAR, ce travail s'est fait en collégialité avec des conseillers de sécurité et des architectes technologiques de I'UL qui ne sont pas rattachés à PULSAR, mais qui ont une vue d'ensemble de l'infrastructure technologique de l'UL.

Le tableau 3 présente une synthèse des bonnes pratiques/actions organisationnelles déployées chez PULSAR 
afin de favoriser la création de valeur lors du cycle d'innovation dans des écosystèmes d'IO. 


\section{Tableau 3. Synthèses des bonnes pratiques/actions organisationnelles déployées chez PULSAR}

Bonnes pratiques/actions organisationnelles

BP1 Favoriser une culture d'ouverture et d'expérimentation

BP2 Impliquer les partenaires à l'aide de plateformes collaboratives et coordonner les échanges

BP3 Générer et collecter des idées à l'aide d'une plateforme numérique

BP4 Développer une présence sur les réseaux sociaux impliqués clés d'IO

BP6 Cartographier les besoins et les capacités des utilisateurs finaux

BP7 Développer des prototypes et des prétests fréquemment

BP8 Adopter une approche agile

\section{Manifestations chez Pulsar}

La responsable du projet a toujours accueilli les partenaires qui souhaitaient s'investir dans PULSAR et elle a surtout maintenu une atmosphère de collégialité, d'ouverture et de transparence avec tous les partenaires. Les exigences en sécurité technologique ont été établies en collégialité avec des conseillers de sécurité et des architectes technologiques de I'UL qui ne sont rattachés à PULSAR.

L'équipe de PULSAR a organisé des séances de brainstorming, à distance ou en présentiel, avec tous les partenaires afin d'avoir la plus grande variété d'idées et d'aller chercher l'adhésion.

PULSAR veut rapprocher les chercheurs, les décideurs, les intervenants en santé et les citoyens afin que la connaissance développée soit utilisée par les décideurs pour en faire bénéficier les citoyens. PULSAR se veut donc un écosystème apprenant.

L'équipe de PULSAR a organisé des séances de brainstorming, à distance ou en présentiel, avec tous les partenaires afin d'avoir la plus grande variété d'idées et d'aller chercher l'adhésion.

Par exemple, un atelier de brainstorming a été organisé où cinq noms ont été retenus et, par la suite, un vote a été tenu auprès de tous les partenaires.

PULSAR utilise sur son site Web ainsi que sur les médias sociaux pour diffuser de l'information en lien avec des dizaines d'évènements liés à la santé durable et y publie une vingtaine d'articles accessibles. Cette diffusion permet également à PULSAR de faire de la vulgarisation scientifique, de partager les bons coups ainsi que de rassembler la communauté d'organisations et d'individus intéressés et interpellés par les enjeux de santé durable.

PULSAR utilise des outils de communication numérique afin d'ouvrir ses portes pour : présenter et attirer d'autres projets de recherche, valoriser les données et stimuler la recherche.

De grandes « messes » regroupant parfois près de 150 partenaires de différents milieux ont été organisées afin de faire des séances d'idéation.

Identifier, cartographier et analyser l'ensemble des parties prenantes de l'écosystème PULSAR afin de répertorier les expertises, les capacités et les besoins de l'ensemble des parties prenantes.

Tout d'abord avoir un mindset client signifie : bien identifier le ou les clients existants et potentiels de PULSAR, tenter de se mettre dans la peau de ses clients et ramener constamment ces derniers au cœur de toutes les discussions.

L'équipe de PULSAR a présenté des projets qui n'ont pas été retenus pour les autorités en place. Par contre, ces projets ont tout de même permis de tester des idées, d'avoir de la rétroaction et de faire émerger d'autres idées de projets qui eux ont réussi.

La philosophie de PULSAR est que l'apprentissage organisationnel à chaque projet est transférable vers le prochain projet.

Les principes d'innovation et d'agilité ont guidé le développement de l'écosystème PULSAR c.-à-d. faire des sprints d'un ou deux mois, faire de petites livraisons fréquentes, collecter les rétroactions et se réajuster. 


\begin{tabular}{|c|c|c|}
\hline & & $\begin{array}{l}\text { - Il n'y avait pas d'anticipation, pas de plan exhaustif, pas d'analyse très détaillée, mais tout était structuré, un } \\
\text { peu comme dans la méthode Scrum. L'équipe PULSAR avançait par itérations en identifiant les besoins et en } \\
\text { travaillant sur les priorités. } \\
\text { - L'équipe de PULSAR a eu à créer la structure et celle-ci a évolué au fur et à mesure avec les projets et leurs } \\
\text { besoins. Chez PULSAR, il n'y a toujours pas de structure finale. Elle est en constante évolution et en adaptation. } \\
\text { - Commencer par livrer ce qui donnait le " plus de valeur ", ce qui était prioritaire en fonction des projets des } \\
\text { chercheurs proposés. } \\
\text { - PULSAR opère avec une approche par petits pas en partant de son noyau et en construisant autour. } \\
\text { - L'équipe de PULSAR dispose également d'un état d'esprit qui accepte que tout ce qui sera fait ne sera pas } \\
\text { parfait, car il est impossible de tout prévoir, ce qui est contraire à la philosophie scientifique qui s'attarde sur les } \\
\text { détails qui deviennent souvent des freins. }\end{array}$ \\
\hline BP9 & $\begin{array}{l}\text { Gérer les enjeux relatifs à la gouvernance et la } \\
\text { sécurité des données }\end{array}$ & $\begin{array}{l}\text { - Afin de respecter les exigences éthiques, légales et technologiques entourant le dépôt, le partage et l'utilisation } \\
\text { de ces données, plusieurs protocoles d'approbation ont été développés et mis en place pour assurer l'intégrité, } \\
\text { la sécurité et la qualité des données. }\end{array}$ \\
\hline BP10 & $\begin{array}{l}\text { Promouvoir une communication ouverte et } \\
\text { transparente entre les partenaires }\end{array}$ & $\begin{array}{l}\text { - Ce principe de co-construction, qui est au cœur même du fonctionnement de l'écosystème, a également été } \\
\text { utilisé pour développer, en toute transparence, la vision/mission de PULSAR avec les partenaires. }\end{array}$ \\
\hline BP11 & $\begin{array}{l}\text { Renforcer la motivation et l'engagement des } \\
\text { partenaires }\end{array}$ & $\begin{array}{l}\text { - Mieux vaut focaliser sur les partenaires « positifs », ainsi que sur les « neutres » plutôt que perdre son temps, } \\
\text { son attention et son énergie avec les partenaires « négatifs ». } \\
\text { - Un chercheur-parrain a été mandaté pour mener les activités en lien avec chaque thème. }\end{array}$ \\
\hline BP12 & $\begin{array}{l}\text { Allouer des ressources financières suffisantes aux } \\
\text { initiatives d'IO }\end{array}$ & $\begin{array}{l}\text { - Un comité directeur a été formé et a permis à PULSAR d'aller chercher du support à trois niveaux : 1) Politique, } \\
\text { 2) Recherche, et 3) Financier. }\end{array}$ \\
\hline BP13 & Obtenir un support des hauts dirigeants & $\begin{array}{l}\text { - Former un comité directeur pour aller chercher du support à trois niveaux : 1) Politique, 2) Recherche, et 3) } \\
\text { Financier. }\end{array}$ \\
\hline BP14 & $\begin{array}{l}\text { Développer un leadership fort et une vision claire et } \\
\text { cohérente }\end{array}$ & $\begin{array}{l}\text { - Établir un mandat clair, simple et qui tenait en une seule phrase : Mettre en place une plateforme collaborative } \\
\text { de recherche et d'intervention en santé durable. } \\
\text { - Mobiliser les partenaires et acteurs afin d'établir une définition commune et opérationnelle du concept de } \\
\text { santé durable. } \\
\text { - Élaborer un cadre d'évaluation de la santé durable afin de/d : 1) clarifier le concept de santé durable, 2) } \\
\text { partager des expertises et des connaissances, 3) initier de nouvelles collaborations, et 4) élargir la portée et la } \\
\text { collecte de données de certains projets. }\end{array}$ \\
\hline BP15 & $\begin{array}{l}\text { Identifier et sélectionner des membres d'équipe } \\
\text { avec des compétences complémentaires et une } \\
\text { bonne attitude }\end{array}$ & $\begin{array}{l}\text { - La détermination, l'engagement, le dévouement et l'attitude positive des membres de l'équipe sont des } \\
\text { caractéristiques essentielles. } \\
\text { - PULSAR compte sur une équipe de } 17 \text { personnes possédant des expériences et des expertises variées et } \\
\text { complémentaires. } \\
\text { - PULSAR compte également sur l'expertise et les conseils de quatre comités soit un comité directeur, un comité } \\
\text { scientifique, un comité de partenaire et un comité des représentants du public. }\end{array}$ \\
\hline BP16 & Utiliser un cadre de travail collaboratif commun & $\begin{array}{l}\text { - L'un des principes clés de gestion chez Pulsar a toujours été l'hyper collaboration qui se résume ainsi : Si, en } \\
\text { tant qu'individu ou organisation, tu ne collabores pas à l'objectif commun de PULSAR, si tu ne veux pas }\end{array}$ \\
\hline
\end{tabular}




\begin{tabular}{|c|c|c|}
\hline & & $\begin{array}{l}\text { t'impliquer, pas de soucis. PULSAR continue à avancer uniquement avec ceux et celles qui souhaitent } \\
\text { s'impliquer et travailler pour l'objectif commun. Tu te mets sur le côté et tu embarqueras lorsque tu } \\
\text { souhaiteras t'investir et t'impliquer. } \\
\text { - PULSAR réalise des projets collaboratifs et interdisciplinaires regroupant des chercheurs de disciplines très } \\
\text { variées, ainsi que des décideurs, des citoyens et/ou des intervenants en santé. La mise en commun des } \\
\text { connaissances en santé durable et l'esprit de partage sont au cœur même de la mission de PULSAR. }\end{array}$ \\
\hline BP17 & Surveiller le déroulement des initiatives d'IO & $\begin{array}{l}\text { - Ces quatre éléments guident l'ensemble des actions des partenaires de l'écosystème PULSAR ainsi que le } \\
\text { déroulement des différentes initiatives liées à PULSAR. Ces éléments jouent le rôle de « contrat moral » entre } \\
\text { les partenaires. } \\
\text { - Puisqu'il y avait plusieurs groupes de parties prenantes avec des demandes/exigences divergentes, l'équipe de } \\
\text { PULSAR a dû prioriser. Il a été décidé de débuter avec le volet recherche et les chercheurs. } \\
\text { - Les nouveaux projets devaient par la suite être présentés et validés avec les autres projets et les partenaires de } \\
\text { PULSAR avant d'être réalisés. }\end{array}$ \\
\hline BP18 & $\begin{array}{l}\text { Déployer une infrastructure informatique flexible et } \\
\text { des données ouvertes }\end{array}$ & $\begin{array}{l}\text { - Utilise le numérique et l'intelligence collective (engagement et mobilisation citoyens) pour trouver des solutions } \\
\text { concrètes au bénéfice de la santé et du bien-être des collectivités. L'offre de services de PULSAR se distingue } \\
\text { par l'adoption des principes FAIR de gestion et d'intendance des données (Facilement trouvables, Accessibles, } \\
\text { Interopérables, Réutilisables). } \\
\text { - PULSAR propose une offre de service technologique favorisant le partage scientifique et la réutilisation des } \\
\text { données, le travail en interdisciplinarité - intersectorialité, les retombées concrètes pour le citoyen, une } \\
\text { démarche éthique et l'engagement du citoyen. } \\
\text { - L'idée de la BDSD est de favoriser la valorisation des données qui y sont déposées et réutilisées dans le futur } \\
\text { dans le cadre d'autres projets, par exemple à des fins de croisements et d'analyses. } \\
\text { - De nouveaux outils d'analyses pourraient émerger, de nouveaux besoins pourraient émerger et ces « data } \\
\text { lake " pourraient être utiles. Toutes ces données sont disponibles aux partenaires qui suivent les protocoles } \\
\text { d'approbation de PULSAR. }\end{array}$ \\
\hline \multicolumn{3}{|c|}{ Bonnes pratiques complémentaires identifiées chez PULSAR } \\
\hline & $\begin{array}{l}\text { Obtenir l'appui de tous les partenaires lors de } \\
\text { décisions majeures ou stratégiques en lien avec } \\
\text { l'objectif commun }\end{array}$ & $\begin{array}{l}\text { - Aucune décision majeure ou stratégique, en lien avec l'objectif commun, ne s'est prise sans avoir consulté et } \\
\text { avoir l'appui des différents comités de partenaires scientifiques, d'affaires et de citoyens. }\end{array}$ \\
\hline & $\begin{array}{l}\text { Analyser les parties prenantes + Établir carte } \\
\text { politique et enjeux }\end{array}$ & $\begin{array}{l}\text { - Identifier, cartographier et analyser l'ensemble des parties prenantes de l'écosystème PULSAR afin de tracer la } \\
\text { carte « politique » du projet ainsi que d'identifier les enjeux actuels et à venir. }\end{array}$ \\
\hline & $\begin{array}{l}\text { Identifier et prioriser les opportunités à supporter et } \\
\text { à mettre de l'avant }\end{array}$ & $\begin{array}{l}\text { - PULSAR baigne dans une mer d'opportunités et avance par opportunité. La clé est de savoir comment choisir les } \\
\text { opportunités à prioriser, c'est-à-dire celles qui sont alignées avec les objectifs et la vision de PULSAR. }\end{array}$ \\
\hline
\end{tabular}


La CISAM repose sur les connaissances et les ambitions partagées par quatre organisations fondatrices : Aix-Marseille Université (AMU), la métropole Aix-Marseille ainsi que deux multinationales : la CMA

CGM et le Groupe L'OCCITANE

\subsubsection{CISAM - Description, structure et objectifs}

Une seconde étude de cas a été réalisée en France avec la Cité de l'innovation et des savoirs Aix-Marseille (CISAM) ${ }^{65}$, qui est un espace unique de créativité entrepreneuriale, de rencontres et d'enrichissement sur le territoire d'Aix-Marseille (France). La CISAM repose sur les connaissances et les ambitions partagées par quatre organisations fondatrices: Aix-Marseille Université ${ }^{66}$ (AMU), la métropole Aix-Marseille ${ }^{67}$ ainsi que deux multinationales : la CMA CGM ${ }^{68}$ et le Groupe L'OCCITANE ${ }^{69}$.

Aix-Marseille Université, qui est la plus grande université francophone au monde, participe à la création de richesses économiques et sociales par l'entremise de l'innovation et de la valorisation de la recherche effectuée dans ses murs. Ces recherches peuvent se traduire par l'émergence de nouveaux débouchés, par la création d'emplois ou encore par l'établissement de nouvelles collaborations. Effectivement, AMU possède plus de 120 structures de recherche (p. ex. laboratoires, instituts, centres, etc.) couvrant un très large éventail de thématiques dont le potentiel d'innovation et de rayonnement est considérable. Or, l'un des défis pour AMU et ses structures de recherche est de rapprocher les acteurs des mondes universitaire, institutionnel et socio-économique, autour des projets communs et fédérateurs. L'objectif de la CISAM est de développer, au sein des acteurs privés et publics du territoire d'Aix-Marseille, une culture scientifique (la science étant vectrice d'innovation) et d'innovation ouverte afin de supporter et stimuler l'essor économique de la région.

Bâtiment et écosystème - La CISAM a donc été créée afin de faciliter les rapprochements entre tous les acteurs de l'innovation, actuels et futurs, au service du territoire de la métropole Aix-Marseille. Inaugurée en mars 2019, la CISAM est tout d'abord un bâtiment de 3000 mètres carrés qui regroupe

\footnotetext{
65 https://cisam-innovation.com/

66 https://www.univ-amu.fr/

${ }^{67}$ https://www.ampmetropole.fr/

68 https://www.cma-cgm.fr/

69 https://group.loccitane.com/fr
} 
L'objectif principal de la CISAM est de devenir le guichet unique sur le territoire d'Aix-Marseille pour faciliter et accélérer

les mises en relations entre tous les acteurs, privés ou publiques, de

l'innovation avec

l'ensemble des chercheurs et expertises de pointes disponibles dans les structures de recherche $d^{\prime} A M U$ sous un même toit plusieurs acteurs clés de l'écosystème d'innovation de la métropole, soit : Obratori ${ }^{70}$, I'incubateur de L'Occitane; ZeBox ${ }^{71}$, l'incubateur du groupe CMA CGM ; l'accélérateur $M^{72}$ de la Métropole Aix-Marseille-Provence, le tout supporté par AMU. Ce bâtiment se veut un lieu totem, un espace au service de tous les individus et organisations qui souhaitent innover sur le territoire d'Aix-Marseille. La CISAM et son bâtiment souhaitent faciliter la tenue de rencontres et d'ateliers, favoriser les collaborations, encourager l'engagement des partenaires, promouvoir une communication ouverte et fluide, stimuler la création, ainsi qu'incuber et accélérer des projets d'innovation. La CISAM aspire à devenir un pôle d'activité économique incontournable et un point de convergence pour connecter et soutenir les acteurs clés de l'écosystème d'Aix-Marseille. La CISAM est donc à la fois un lieu de rencontres, d'échanges, d'idéation, de création, d'incubation de projets, et d'accélération.

Guiche unique, partenaires et pratiques - La CISAM a été mise sur pied afin de réunir des acteurs privés et publics pour faciliter I'innovation au sein d'un lieu unique de créativité entrepreneuriale. L'objectif principal de la CISAM est de devenir le guichet unique sur le territoire d'Aix-Marseille pour faciliter et accélérer les mises en relations entre tous les acteurs, privés ou publiques, de l'innovation avec l'ensemble des chercheurs et expertises de pointe disponibles dans les structures de recherche d'AMU. La CISAM souhaite alléger le travail des partenaires et accélérer l'avancement des innovations.

En plus des quatre organisations fondatrices de la CISAM, cette dernière rassemble également dans son giron des partenaires et acteurs clés de l'innovation et de la recherche publique $: 1$ ) la direction de la recherche et de la valorisation (DRV) d'AMU ${ }^{73}$,

\footnotetext{
70 https://obratori.com/

${ }^{71}$ https://www.ze-box.io/

72 https://accelerateurm.com/

73 La DRV soutient les unités de recherche d'AMU et leurs personnels dans toutes les démarches nécessaires au montage de projets de recherche publique et suit leur
} 


\section{La CISAM représente donc}

un terrain

\section{d'expérimentation pour}

AMU en ce sens que de

nouveaux modèles

d'affaires ont été testés et

déployés, et ce, en

concertation et avec

l'appui de différentes

directions d'AMU
2) Protisvalor ${ }^{74}$, la 3) SATT Sud-Est ${ }^{75}$, 4) I'Institut Carnot STAR ${ }^{76}$, ainsi que les incubateurs 5) Impulse et 6) Belle de Mai ${ }^{77}$.

La CISAM représente donc, pour AMU et ses partenaires, un concept organisationnel novateur au service de l'innovation. Cette structure, qui est unique dans le paysage universitaire français, a permis à AMU et à ses structures de recherche de revoir ses processus et pratiques. La CISAM représente donc un terrain d'expérimentation pour $\mathrm{AMU}$ en ce sens que de nouveaux modèles d'affaires ont été testés et déployés, et ce, en concertation et avec l'appui de différentes directions d'AMU. Par exemple, la CISAM qui est, par l'entremise d'AMU, le locataire principal des locaux aux partenaires, a dû revoir ses pratiques de mutualisation de lieux ainsi que de partage d'équipements et de services, afin de les rendre accessibles et disponibles à l'ensemble des usagers et partenaires. De plus, les espaces de travail ont été repensés afin de favoriser l'ouverture, la collaboration et la créativité, comme, par exemple : bureaux en open space, équipements modulables, espaces de rencontre informels, espace de coworking, etc. L'objectif derrière ces nouveaux processus et ces nouvelles pratiques est de favoriser la transversalité, l'échange d'idées et l'éclosion de nouveaux projets.

exécution, https://www.univ-amu.fr/fr/public/direction-de-la-recherche-et-de-lavalorisation-drv.

74 Protisvalor, qui est une filiale d'AMU, accompagne les chercheurs pour valoriser leurs compétences et leurs résultats, dans les domaines administratif, financier, juridique et de la propriété intellectuelle. Elle assure plus le suivi et la préparation des contrats, https://www.protisvalor.com/site/fr .

75 Le rôle de la SATT Sud-Est est d'aider à maximiser l'impact socio-économique des résultats de la recherche académique et le transfert de technologies. Cette organisation développe et transfère les résultats de recherche des laboratoires publics vers le monde socio-économique en plus de protéger les acteurs par la propriété intellectuelle.

${ }^{76}$ Le rôle de l'Institut Carnot STAR est de favoriser la recherche partenariale entre les secteurs publics et privés dans les domaines du sport, de la santé et du bien-être, en plus d'accélérer et de faciliter les transferts de connaissances et de technologies vers les acteurs socio-économiques. https://www.instituts-carnot.eu/fr/institutcarnot/star

77 Impulse (http://incubateur-impulse.fr/) est un incubateur généraliste qui accompagne le lancement de projet d'entrepreneuriats, alors que Belle de Mai (https://www.belledemai.org/) est un incubateur spécialisé en multimédia. 
La CISAM veut jouer un rôle structurant dans le "parcours de l'innovation »
Parcours de l'innovation - Afin d'accompagner celles et ceux qui innovent sur le territoire d'Aix-Marseille, la CISAM veut jouer un rôle structurant dans le "parcours de l'innovation ", c'est-à-dire du dépôt initial d'un projet, d'une idée ou d'un concept jusqu'à sa mise sur le marché. L'un des rôles clés joués par la CISAM est d'accroître la visibilité des travaux des laboratoires, de les mettre en valeur, et de favoriser l'émergence de partenariats entre chercheurs et acteurs privés et/ou publics. Comme l'illustre la figure 6 , le parcours de l'innovation se découpe en quatre grandes étapes: 1) Laboratoires de recherche, 2) Structures de valorisation et de transfert technologique, 3) Incubateurs et 4) Accélérateurs.

\section{Figure 6. CISAM - Intégralité du parcours de l'innovation}
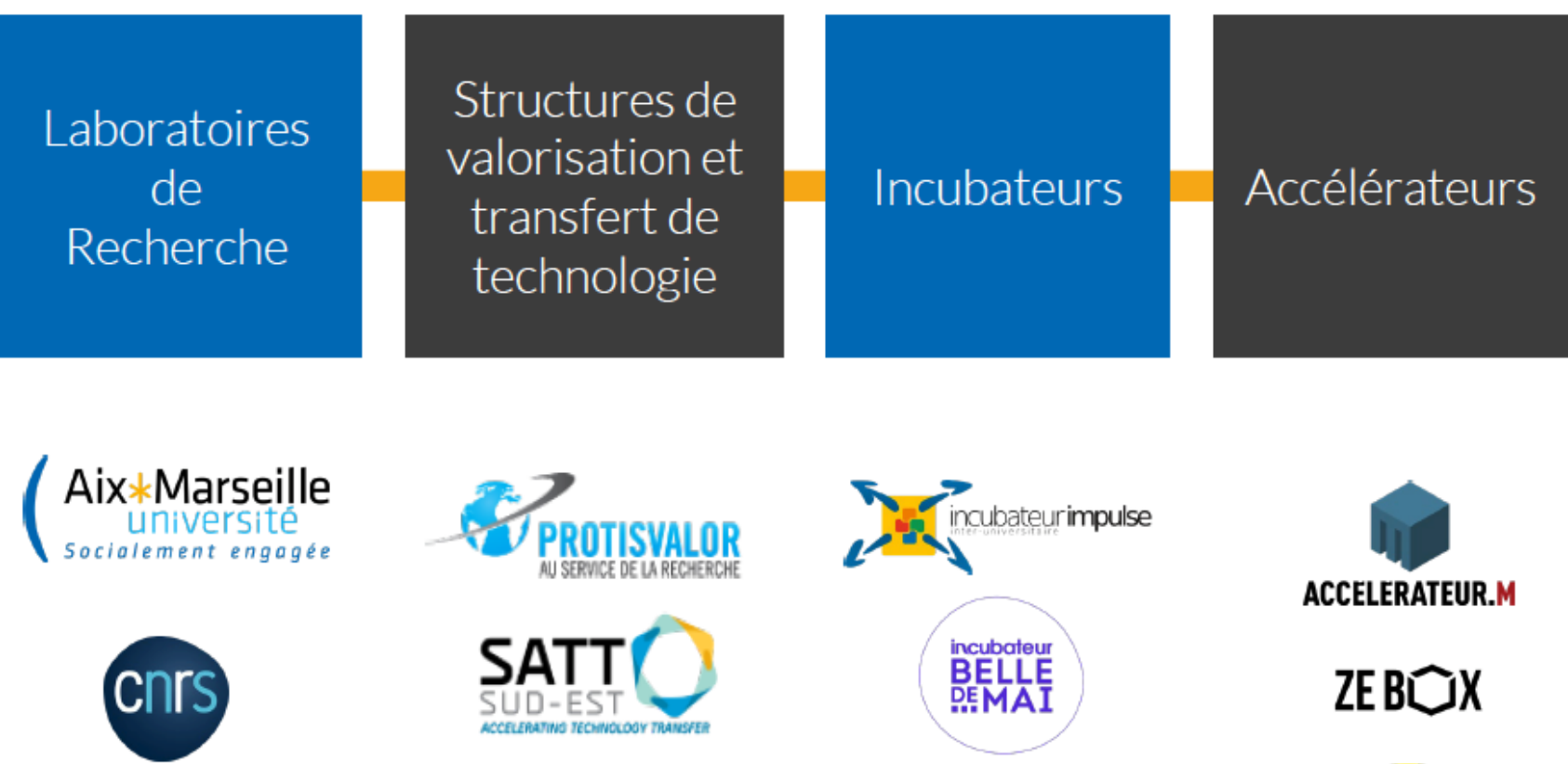

槛 Inserm

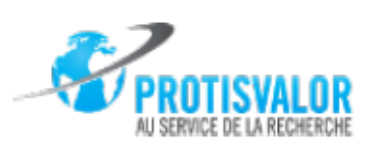

SATT

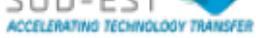

INSTITUT

SIAR
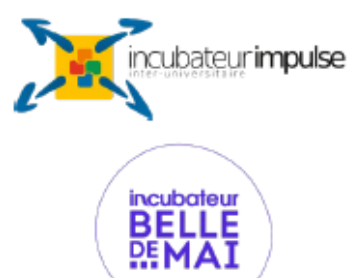

BELLE

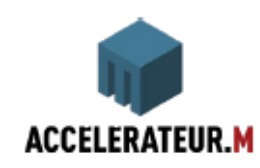

ZEB⿵人X

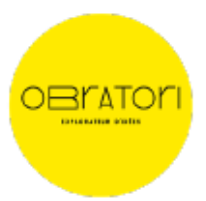

Tel qu'illustré dans la figure 7, ce parcours de l'innovation est directement aligné avec les différentes étapes du parcours de 
la valorisation de la recherche publique qui s'étendent de la recherche fondamentale aux développements industriels en passant par la recherche appliquée.

\section{Figure 7. CISAM - Parcours de la valorisation de la recherche publique}

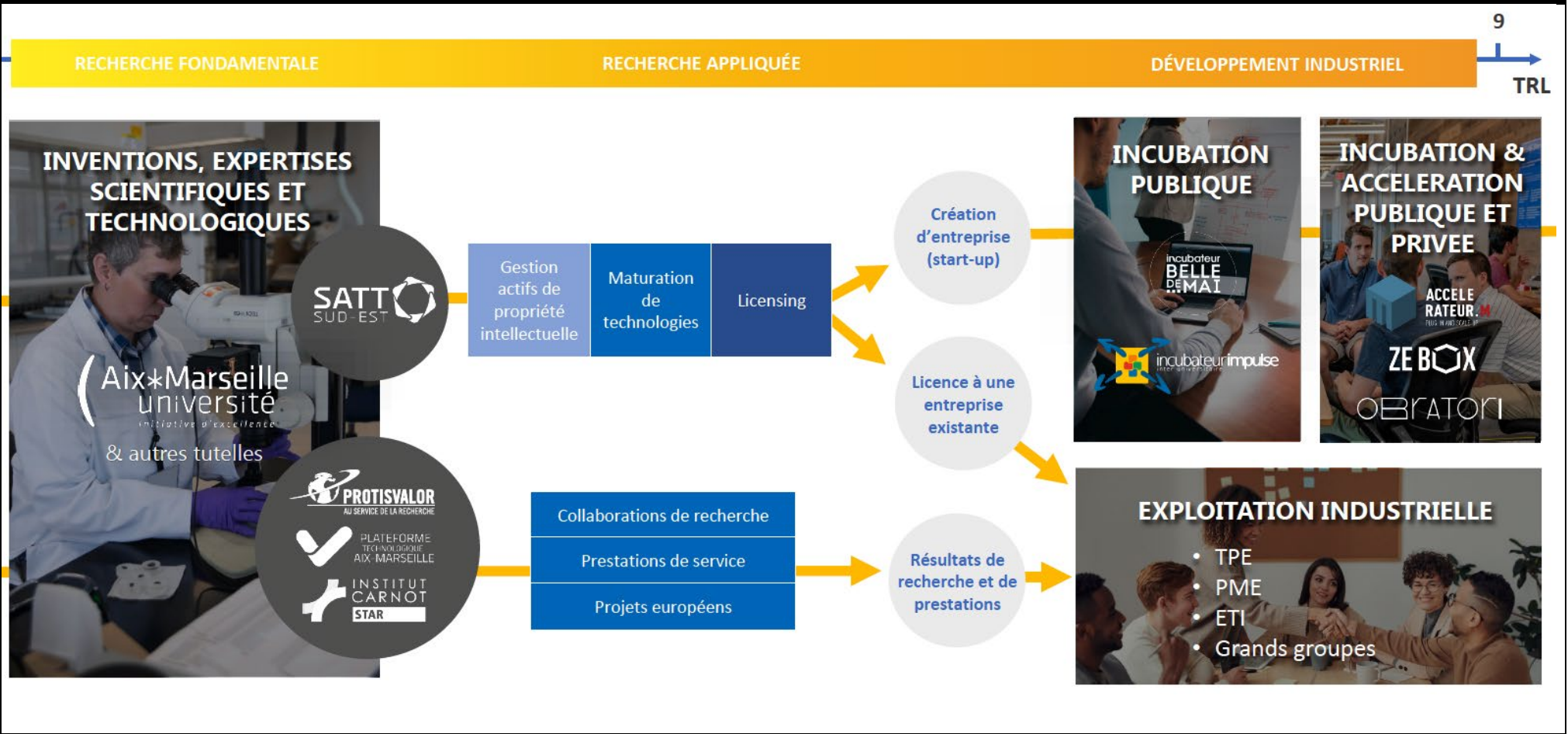

CISAM souhaite stimuler, fluidifier et accélérer les démarches innovantes par l'entremise de services adaptés à chaque projet
Effectivement, la CISAM souhaite stimuler, fluidifier et accélérer les démarches innovantes par l'entremise de services adaptés à chaque projet, comme, par exemple :

1. Organiser des événements et faciliter le networking,

2. Offrir du coaching, de l'expertise et du mentorat,

3. Supporter et stimuler la co-innovation,

4. Accueillir physiquement des équipes de travail,

5. Fournir des conseils en ressources humaines,

6. Accompagner aussi bien les acteurs que les chercheurs dans le processus d'innovation, et ce peu importe le niveau de maturité technologique ${ }^{78}$,

78 https://www.ic.gc.ca/eic/site/080.nsf/fra/00002.html 
Afin d'assurer l'implication

des principaux

partenaires, de coordonner, d'aligner et

de concrétiser les activités

et les projets, de faciliter

les échanges et de

favoriser le travail

collaboratif, une structure

et des processus

novateurs, simples

flexibles et dynamiques

ont été mis en place.
7. Aider dans la recherche de financements et dans les dépôts de brevets,

8. Simplifier et accélérer l'établissement de contrat avec les unités de recherche et les acteurs socioéconomiques,

9. Faciliter le développement d'affaires.

La CISAM peut offrir ces services de support et d'accompagnement, car elle est connectée avec les trois groupes d'acteurs clés dans l'écosystème d'innovation du territoire d'Aix-Marseille, soit les : 1) Talents, par exemple, via le recrutement d'étudiants, de postdoctorants, de spécialistes, etc., via l'encadrement de thèses $\operatorname{CIFRE}^{79}$; via la tenue de challenges/concours/hackatons étudiants, etc.; 2) Laboratoires de recherche, par exemple, via la mise en relation qualifiée avec les laboratoires et plateformes technologiques (prestations et collaborations de recherche), la recherche de brevets issus des laboratoires ou encore la formation continue ; et 3) Acteurs du développement, c.-à-d. par exemple par les relations directes et continues avec les incubateurs, les accélérateurs, les pôles de compétitivités, les industriels, les acteurs du financement ou encore les structures de valorisation et transferts technologiques.

Fonctionnement, comités et équipes - Afin d'assurer l'implication des principaux partenaires, de coordonner, d'aligner et de concrétiser les activités et les projets, de faciliter les échanges et de favoriser le travail collaboratif, une structure et des processus novateurs, simples flexibles et dynamiques ont été mis en place. Au niveau stratégique, un comité de pilotage, réunissant les quatre principaux partenaires, a été créé. Ce comité, qui se réunit une fois par mois, joue un rôle central dans l'écosystème de la CISAM, car il a la responsabilité

\footnotetext{
79 « Renforcer les échanges entre les laboratoires de recherche publique et les milieux socio-économiques, favoriser l'emploi des docteurs dans les entreprises et contribuer au processus d'innovation des entreprises établies en France, telle est la vocation des Conventions industrielles de formation par la recherche (CIFRE) ", Source : https://www.enseignementsup-recherche.gouv.fr/fr/les-cifre-46510
} 
Lorsque la CISAM a été lancée, la vision du projet était de faciliter et d'encourager l'innovation à l'aide de deux éléments clés : un lieu physique et un partenariat public privé d'établir les grandes orientations de la CISAM ainsi que d'examiner, d'évaluer et de sélectionner les projets et les actions qui seront déployés. Au niveau opérationnel, un comité de suivi a été créé afin de maintenir des relations efficaces et harmonieuses entre les partenaires et l'ensemble des acteurs œuvrant avec la CISAM, de veiller au bon fonctionnement du bâtiment de la CISAM et de réagir rapidement aux opportunités et problématiques se présentant. Par ailleurs, des groupes de travail thématiques ont été créés afin d'aborder et de répondre aux questions plus spécifiques ou ponctuelles. Présentement, la CISAM fonctionne grâce à une petite équipe de cinq personnes dynamiques et dévouées: un directeur, une responsable administrative et financière, un gestionnaire administratif, une chargée de communication ainsi qu'un "business developer». De par sa taille et la qualité des membres de l'équipe, ainsi que de par la nature même des activités de la CISAM, l'équipe de la CISAM est très agile et flexible. Par ailleurs, l'équipe de la CISAM a adopté un esprit de startup. Elle peut répondre très rapidement aux opportunités qui se présentent à elles. II faut mentionner que la structure de la CISAM est plus légère que les structures complexes d'AMU et l'écosystème d'innovation en place.

\subsubsection{CISAM - Pratiques déployées pour favoriser l'innovation ouverte}

Vision et bâtiments - Lorsque la CISAM a été lancée, la vision du projet était de faciliter et d'encourager l'innovation à l'aide de deux éléments clés : un lieu physique et un partenariat public-privé. Tout d'abord, le bâtiment totem de la CISAM se veut un message fort de l'importance de cette initiative et surtout un lieu symbolique de rencontres pour échanger des idées et développer des relations. D'importantes ressources financières ont été investies par AMU et ses partenaires pour concrétiser cette vision et donner une impulsion et une crédibilité à ce projet. De plus, en rapprochant, aussi bien physiquement qu'au niveau des idées, les chercheurs, les 


\section{Il est particulièrement}

important d'établir une compréhension commune entre langage commercial et celui parlé par les chercheurs, car les priorités, les références, les contextes et les objectifs sont différents. étudiants, les entrepreneurs et les acteurs socio-économiques dans ce lieu, la CISAM a souhaité créer un espace pour faciliter l'innovation et stimuler la créativité entrepreneuriale. En regroupant sous un même toit et en liant dans un partenariat public-privé, quatre joueurs clés, c.-à-d. AMU, l'Accélérateur M (Métropole), ZEBOX (CMA CGM) et OBRATORI (L'Occitane), la CISAM a réussi à réunir à un seul endroit un portefeuille de ressources, d'expertises, de connaissances et de services qui sont mis à la disposition des individus et des organisations souhaitant innover dans la métropole Aix-Marseille.

Afin de développer cette vision commune et des objectifs communs, des groupes de travail ont été mis en place avec les partenaires et les acteurs clés de la métropole afin de développer une vision commune, d'expliquer et clarifier les rôles de chacun, établir un mode de fonctionnement commun en plus de développer un langage commun. Effectivement, il est particulièrement important d'établir une compréhension commune entre le langage commercial et celui parlé par les chercheurs, car les priorités, les références, les contextes et les objectifs sont différents. La CISAM joue un rôle de sensibilisation de ces différences entre les intervenants.

La volonté de la CISAM est de répondre le plus rapidement possible aux besoins des industriels et aux opportunités d'affaires en fournissant des ressources critiques aux entreprises et porteurs de projets et en mettant en réseau tous les acteurs et les lieux de l'innovation. D'ailleurs, la CISAM doit aider ces acteurs à optimiser et garantir la continuité du financement.

Il faut également noter que la CISAM a été lancée à un moment où un très grand nombre d'initiatives de support à l'innovation existaient dans la métropole, sans vraiment se parler. L'une des missions de la CISAM était, entre autres, de fédérer ces initiatives sous un même toit physique, numérique et symbolique.

Benchmarking d'initatives similaires - Avant de lancer la CISAM, un important exercice de benchmarking a été effectué 
Cet exercice a été effectué en faisant valoir les complémentarités, en expliquer les différences entre accélérateur et incubateur, en présentant le parcours de l'innovation, en tissant des liens avec les individus et surtout martelant le message que le rôle principal de la CISAM est de fluidifier les relations entre les acteurs de l'innovation. par le directeur de CISAM et son équipe. Afin de bien positionner la CISAM et développer une vision claire et précise, des structures similaires, comme, par exemple, Station $\mathrm{F}^{80}$ (Paris), Quartier de l'innovation ${ }^{81}$ (Montréal), MIT Node ${ }^{82}$ (Hong Kong) ou encore Jurong Innovation District ${ }^{83}$ (Singapore), ont été analysées. Cet exercice de benchmarking a permis à l'équipe de la CISAM d'identifier les bonnes pratiques, d'identifier ses " concurrents " et d'adapter sa vision et son offre au contexte et aux acteurs de la métropole d'AixMarseille.

Rôles de business développeur - Afin de mener à bien sa mission première qui est de mettre en relation tous les acteurs de la métropole d'Aix-Marseille afin de stimuler l'innovation, la CISAM a embauché des "business developers ". Lors de la première année d'opération de la CISAM, cinq business developers supplémentaires ont été embauchés. Leurs rôles étaient de faire connaître la CISAM, cartographier les expertises dans les structures de recherche d'AMU, et de favoriser les mises en relation entre les chercheurs et les acteurs socioéconomiques.

Dès le lancement de la CISAM, I'un des défis a été de se faire connaître à l'intérieur même d'AMU en clarifiant le rôle de la CISAM et de ses services auprès des chercheurs et des étudiants, en clarifiant les enjeux de gouvernance avec les structures existantes, en facilitant l'établissement de liens entre la structure de recherche et la pratique ainsi qu'en valorisant la transversalité et la complémentarité des expertises. Puisque la CISAM venait s'ajouter à un écosystème d'innovation complexe déjà en place à l'AMU, beaucoup d'efforts ont été déployés pour expliquer et clarifier les rôles et les distinctions de la CISAM par rapport aux acteurs en place. Cet exercice a été effectué en faisant valoir les complémentarités, en expliquant les différences entre

\footnotetext{
${ }^{80} \mathrm{https}$ ://stationf.co/

${ }^{81}$ https://quartierinnovationmontreal.com/

82 https://hkinnovationnode.mit.edu/

${ }^{83}$ https://estates.jtc.gov.sg/jid
} 
La cartographie des compétences des équipes de recherche à $A M U, c .-\grave{a}-$

d. plus de 4500 enseignants-chercheurs et 122 laboratoires, afin de pouvoir filtrer les futures demandes provenant d'acteurs socioéconomiques et bien cibler les experts à consulter. accélérateur et incubateur, en présentant le parcours de l'innovation, en tissant des liens avec les individus et surtout en martelant le message que le rôle principal de la CISAM est de fluidifier les relations entre les acteurs de l'innovation. La CISAM devait également se faire connaitre auprès des groupes industriels en expliquant son positionnement et en présentant ses services.

Cartographier les expertises - Tel que mentionné précédemment, la CISAM joue un rôle de coordonnateur entre les partenaires et l'un de ses objectifs est de rendre visibles l'expertise et les recherches effectuées dans les structures de recherche d'AMU. Pour se faire, l'ensemble des business developers a procédé à la cartographie des compétences des équipes de recherche à $A M U, c$.-à-d. plus de 4500 enseignantschercheurs et 122 laboratoires, afin de pouvoir filtrer les futures demandes provenant d'acteurs socio-économiques et bien cibler les experts à consulter. Cette cartographie permet de ne pas surcharger les partenaires et surtout d'accélérer les mises en relation et parcours d'innovations.

Pour ce faire, à chacun des cinq business developers ont été attitrés une des cinq grandes thématiques: numérique, sciences humaines et sociales, santé et sciences de la vie, physique et mécanique ainsi qu'énergie et environnement. Par la suite, chaque business developers a approché chaque responsable de laboratoire de recherche dans AMU et a travaillé individuellement avec chacun d'entre eux afin de comprendre leurs expertises, leurs projets de recherche, leurs apports et leurs intérêts. Ce travail de longue haleine a permis d'établir des liens de confiance. Afin de rejoindre les laboratoires de recherche, la CISAM a tout d'abord contacté les instituts de recherche (qui regroupent plusieurs laboratoires) pour s'introduire et présenter les avantages de la CISAM.

Valeur ajoutée L'une des idées, des stratégies clés de la CISAM n'est pas d'imposer ses services aux partenaires, aux chercheurs et aux acteurs socio-économiques, mais plutôt de montrer clairement ce qu'est la CISAM, d'expliquer ses services, ses avantages et de laisser les chercheurs et 
L'une des idées, des

stratégies clés de la CISAM

n'est pas d'imposer ses

services aux partenaires,

aux chercheurs et aux

acteurs socio-

économiques, mais plutôt

de montrer clairement ce

qu'est le CISAM,

d'expliquer ses services,

ses avantages et de laisser

les chercheurs et

partenaires venir à eux. partenaires venir à eux. La CISAM doit sensibiliser et prêcher par l'exemple, car elle joue un rôle de support et non un rôle hiérarchique par rapport aux partenaires et acteurs de l'écosystème d'innovation. L'idée est de mettre de l'avant la valeur ajoutée de la CISAM, soit sa double compétence : bien connaître les acteurs socio-économiques et bien connaitre l'écosystème d'AMU et ses chercheurs. Ainsi, la CISAM peut accompagner toutes les organisations souhaitant trouver des solutions à des problématiques très variées, car elle a accès à toutes les expertises d'AMU qui couvre un très large éventail de disciplines. De plus, de par son positionnement, ses connaissances et ses liens, la CISAM peut aider à répondre à des problématiques multidisciplinaires qui nécessitent des maillages entre académiques et acteurs privés. La CISAM met en place des relations, crée des configurations de partenaires/experts spécifiques pour des problématiques/idées identifiées. Les avantages de la CISAM sont qu'elle a accès à l'expertise scientifique, à l'expertise professionnelle ainsi qu'au capital financier. La CISAM sait comment traduire les besoins de l'industrie et identifier les expertises d'AMU requises. De plus, la CISAM peut travailler avec des horizons temporels différents selon les besoins et contraintes des partenaires, par exemple des temps " longs " en recherche versus des temps " courts " dans l'industrie. Bref, la valeur ajoutée de la CISAM découle de ses deux rôles clés soit celui de phare : pour donner de la visibilité aux expertises et aux projets de recherche d'AMU, et celui d'accélérateur : pour guider, mailler et accompagner les acteurs publics et privés dans les phases d'innovation.

Le tableau 4 présente une synthèse des bonnes pratiques/actions organisationnelles déployées à la CISAM afin de favoriser la création de valeur lors du cycle d'innovation dans des écosystèmes d'IO. En observant le tableau 4, on note que la gouvernance des données (BP9) ainsi que la notion de données ouvertes (BP19) semblent jouer un rôle moins marqué dans le déploiement de l'écosystème d'innovation de la CISAM. 


\section{Tableau 4. Analyse des Bonnes pratiques/actions organisationnelles déployées chez CISAM}

Bonnes pratiques/actions organisationnelles

BP1 Favoriser une culture d'ouverture et d'expérimentation

BP2 Impliquer les partenaires à l'aide de plateformes collaboratives et coordonner les échanges

\section{Manifestations chez CISAM}

L'objectif de la CISAM est de développer, au sein des acteurs privés et publics du territoire d'Aix-Marseille, une culture scientifique (la science étant vectrice d'innovation) et d'innovation ouverte afin de supporter et stimuler l'essor économique de la région.

- La CISAM représente donc un terrain d'expérimentation pour AMU en ce sens que de nouveaux modèles d'affaires ont été testés et déployés, et ce, en concertation et avec l'appui de différentes directions d'AMU. - Regrouper sous un même toit plusieurs acteurs clés de l'écosystème d'innovation de la métropole.

- L'objectif principal de la CISAM est de devenir le guichet unique sur le territoire d'Aix-Marseille pour faciliter et accélérer les mises en relation entre tous les acteurs, privés ou publics, de l'innovation avec l'ensemble des chercheurs et expertises de pointe disponibles dans les structures de recherche d'AMU.

Les espaces de travail ont été repensés afin de favoriser l'ouverture, la collaboration et la créativité, comme, par exemple : bureaux en open space, équipements modulables, espaces de rencontre informels, espace de coworking, etc.

- Le bâtiment totem de la CISAM se veut un message fort de l'importance de cette initiative et surtout un lieu symbolique de rencontres pour échanger des idées et développer des relations.

- En rapprochant, aussi bien physiquement qu'au niveau des idées, les chercheurs, les étudiants, les entrepreneurs et les acteurs socio-économiques dans ce lieu, la CISAM a souhaité créer un espace pour faciliter l'innovation et stimuler la créativité entrepreneuriale.

L'idée est de mettre de l'avant la valeur ajoutée de la CISAM, soit sa double compétence : bien connaître les acteurs socio-économiques et bien connaître l'écosystème d'AMU et ses chercheurs.

BP3 Générer et collecter des idées à l'aide d'une plateforme numérique Organiser des événements et faciliter le networking + Supporter et stimuler la co-innovation.

Mise en relation qualifiée avec les laboratoires et plateformes technologiques (prestations et collaborations de recherche).

L'une des missions de la CISAM était, entre autres, de fédérer ces initiatives sous un même toit physique, numérique et symbolique.

BP4 Développer une présence sur les réseaux sociaux La CISAM utilise des plateformes comme Linkedin et Twitter pour diffuser les événements et les réalisations de la CISAM

BP5 Organiser des rencontres entre les partenaires impliqués clés d'IO

BP6 Cartographier les besoins et les capacités des utilisateurs finaux
- En plus des quatre organisations fondatrices de la CISAM, cette dernière rassemble également dans son giron des partenaires et acteurs clés de l'innovation et de la recherche publique

- L'un de ses objectifs est de rendre visibles l'expertise et les recherches effectuées dans les structures de recherche d'AMU.

Cette cartographie permet de ne pas surcharger les partenaires et surtout d'accélérer les mises en relation et le parcours d'innovation.

Chaque business developers a approché chaque responsable de laboratoire de recherche dans AMU et a travaillé individuellement avec chacun d'entre eux afin de comprendre leurs expertises, leurs projets de recherche, leurs apports et leurs intérêts. 


\begin{tabular}{|c|c|c|}
\hline BP7 & $\begin{array}{l}\text { Développer des prototypes et des prétests } \\
\text { fréquemment }\end{array}$ & $\begin{array}{l}\text { - Une cartographie des compétences des équipes de recherche à AMU a été effectuée afin de pouvoir filtrer les } \\
\text { futures demandes provenant d'acteurs socio-économiques et bien cibler les experts à consulter. }\end{array}$ \\
\hline BP8 & Adopter une approche agile & $\begin{array}{l}\text { - La CISAM fonctionne grâce à une petite équipe de cinq personnes dynamiques et dévouées. De par sa taille et la } \\
\text { qualité de membres de l'équipe, ainsi que de par la nature même des activités de la CISAM, l'équipe de la } \\
\text { CISAM est très agile et flexible. Par ailleurs, l'équipe de la CISAM a adopté un esprit de startup. } \\
\text { - La CISAM peut répondre très rapidement aux opportunités qui se présentent à elles. Il faut mentionner que la } \\
\text { structure de la CISAM est plus légère que les structures complexes d'AMU et l'écosystème d'innovation en } \\
\text { place. }\end{array}$ \\
\hline BP9 & $\begin{array}{l}\text { Gérer les enjeux relatifs à la gouvernance et la } \\
\text { sécurité des données }\end{array}$ & $-N A$ \\
\hline BP10 & $\begin{array}{l}\text { Promouvoir une communication ouverte et } \\
\text { transparente entre les partenaires }\end{array}$ & $\begin{array}{l}\text { - La CISAM et son bâtiment souhaitent faciliter la tenue de rencontres et d'ateliers, favoriser les collaborations, } \\
\text { encourager l'engagement des partenaires, promouvoir une communication ouverte et fluide, stimuler la } \\
\text { création, ainsi qu'incuber et accélérer des projets d'innovation. }\end{array}$ \\
\hline BP11 & $\begin{array}{l}\text { Renforcer la motivation et l'engagement des } \\
\text { partenaires }\end{array}$ & $\begin{array}{l}\text { - Le rôle principal de la CISAM est de fluidifier les relations entre les acteurs de l'innovation. } \\
\text { - La CISAM peut aider à répondre à des problématiques multidisciplinaires qui nécessitent des maillages entre } \\
\text { académiques et acteurs privés. }\end{array}$ \\
\hline BP12 & $\begin{array}{l}\text { Allouer des ressources financières suffisantes aux } \\
\text { initiatives d'IO }\end{array}$ & $\begin{array}{l}\text { - D'importantes ressources financières ont été investies par AMU et ses partenaires pour concrétiser cette vision } \\
\text { et donner une impulsion et une crédibilité à ce projet. } \\
\text { - La CISAM a réussi à réunir à un seul endroit un portefeuille de ressources, d'expertises, de connaissances et de } \\
\text { services qui sont mis à la disposition des individus et des organisations souhaitant innover dans la métropole } \\
\text { Aix-Marseille. } \\
\text { - La CISAM doit aider ces acteurs à optimiser et garantir la continuité du financement. } \\
\text { - Les avantages de la CISAM sont qu'elle a accès à l'expertise scientifique, à l'expertise professionnelle ainsi qu'au } \\
\text { capital financier. }\end{array}$ \\
\hline BP13 & Obtenir un support des hauts dirigeants & $\begin{array}{l}\text { - La CISAM repose sur les connaissances et les ambitions partagées par quatre organisations fondatrices : Aix- } \\
\text { Marseille Université (AMU), la métropole Aix-Marseille ainsi que deux multinationales : la CMA CGM et le } \\
\text { Groupe L'OCCITANE. }\end{array}$ \\
\hline BP14 & $\begin{array}{l}\text { Développer un leadership fort et une vision claire et } \\
\text { cohérente }\end{array}$ & $\begin{array}{l}\text { - Lorsque la CISAM a été lancée, la vision du projet était de faciliter et d'encourager l'innovation à l'aide de deux } \\
\text { éléments clés : un lieu physique et un partenariat public-privé. }\end{array}$ \\
\hline BP15 & $\begin{array}{l}\text { Identifier et sélectionner des membres d'équipe } \\
\text { avec des compétences complémentaires et une } \\
\text { bonne attitude }\end{array}$ & $\begin{array}{l}\text { - Lors de la première année d'opération de la CISAM, cinq business developers supplémentaires ont été } \\
\text { embauchés. Leurs rôles étaient de faire connaître la CISAM, cartographier les expertises dans les structures de } \\
\text { recherche d'AMU et de favoriser les mises en relation entre les chercheurs et les acteurs socio-économiques. }\end{array}$ \\
\hline BP16 & Utiliser un cadre de travail collaboratif commun & $\begin{array}{l}\text { - L'un des rôles clés joués par la CISAM est d'accroître la visibilité des travaux des laboratoires, de les mettre en } \\
\text { valeur, et de favoriser l'émergence de partenariats entre chercheurs et acteurs privés et/ou publics. }\end{array}$ \\
\hline BP17 & Surveiller le déroulement des initiatives d'IO & $\begin{array}{l}\text { - La CISAM veut jouer un rôle structurant dans le « parcours de l'innovation », c'est-à-dire du dépôt initial d'un } \\
\text { projet, d'une idée ou d'un concept jusqu'à sa mise sur le marché. } \\
\text { - Accompagner aussi bien les acteurs que les chercheurs dans le processus d'innovation, et ce peu importent le } \\
\text { niveau de maturité technologique. }\end{array}$ \\
\hline
\end{tabular}


BP18 Déployer une infrastructure informatique flexible et

des données ouvertes

NA

Bonnes pratiques complémentaires identifiées à la CISAM

Supporter et accompagner les acteurs d'un

La CISAM peut offrir ces services de support et d'accompagnement, car elle est connectée avec trois groupes

écosystème d'innovation

d'acteurs clés dans l'écosystème d'innovation du territoire d'Aix-Marseille, soit les : 1) Talents, 2) Laboratoires de recherche, et 3) Acteurs du développement.

Développer une compréhension et un langage

- Établir une compréhension commune entre le langage commercial et celui parlé par les chercheurs, car les

commun

priorités, les références, les contextes et les objectifs sont différents. La CISAM joue un rôle de sensibilisation à ces différences entre les intervenants. 


\section{Les 18 bonnes}

pratiques/actions

organisationnelles

permettent aux OSP de

développer des capacités

dynamiques avec succès, des initiatives $d^{\prime} 10$

\section{Conclusion}

Les organisations du secteur public (OSP) sont confrontées à de fortes pressions financières, technologiques, environnementales et structurelles. Afin de s'adapter et répondre à ces forces complexes et continuellement en évolution, les OSP doivent déployer de nouvelles approches innovantes et collaboratives afin de capturer et capitaliser sur les connaissances provenant de l'interne comme de l'externe. En adoptant une perspective de capacités dynamiques et sur la base d'une analyse de 253 études de cas d'initiatives d'IO dans des OSP, notre recherche a permis d'identifier 17 défis reliés au déploiement d'IO pour les OSP et 18 bonnes pratiques/actions organisationnelles pour mettre en œuvre de telles initiatives.

Les 18 pratiques/actions permettent aux OSP de développer des capacités dynamiques et ont été collectivement utilisées pour mettre en œuvre, avec succès, des initiatives d'IO. Notre étude montre également que, pour la plupart de ces pratiques/actions organisationnelles, les $\mathrm{TI}$ jouent un rôle clé en tant qu'outil de support. De plus, les OSP devraient tenir compte du fait que la mise en œuvre des pratiques/actions identifiées (tableau 2) les aidera à développer leurs capacités dynamiques et leur permettra de s'engager avec succès dans les initiatives d'IO. Le développement de capacités dynamiques est un processus continu qui nécessite des efforts. Une fois développées et maintenues, ces capacités peuvent être utilisées, supportées et transférées dans d'éventuels projets d'IO. Cette boucle itérative devrait permettre aux OSP de façonner leur agilité organisationnelle ${ }^{84}$.

Les deux études de cas d'écosystèmes d'innovations, soit le cas PULSAR et le cas CISAM, ont pu démontrer les défis de mettre en place et de supporter de telles structures. La présentation et l'analyse de ces cas ont permis d'illustrer concrètement des

${ }^{84}$ (Sambamurthy et al. 2003) 
manifestations des bonnes pratiques / actions organisationnelles liées aux initiatives d'IO. De plus, ces deux cas ont permis d'identifier des pratiques complémentaires qui pourraient guider de futurs acteurs intéressés pour déployer et supporter des écosystèmes d'innovation, soit 1) Obtenir l'appui de tous les partenaires lors de décisions majeures ou stratégiques en lien avec l'objectif commun, 2) Analyser les parties prenantes + Établir une carte politique et les enjeux de chacun, 3) Identifier et prioriser les opportunités à supporter et à mettre en avant, 4) Supporter et accompagner les acteurs d'un écosystème d'innovation, et 5) Développer une compréhension et un langage commun. 


\section{Annexe 1 - Explications théoriques complémentaires}

\section{Processus d'innovation et l'innovation ouverte}

Le processus d'innovation consiste à améliorer significativement une situation existante en transformant des idées innovantes en solutions concrètes qui seront mises en œuvre dans l'organisation ${ }^{85}$. Afin de favoriser l'innovation dans les organisations, il est important de comprendre comment promouvoir, soutenir et encadrer chaque étape du processus d'innovation. Ainsi, pour stimuler et soutenir l'innovation, les organisations doivent mettre en œuvre un ensemble de pratiques/actions par le déploiement et l'utilisation

Les initiatives d'innovation ouverte (IO) permettent $d e / d^{\prime}$ :

1) Améliorer la vitesse du cycle d'innovation,

2) Diversifier les sources d'innovation, d'expertise et de connaissances,

3) Capturer l'intelligence collective. d'infrastructures et de ressources organisationnelles, financières et technologiques ${ }^{86}$.

La mise en œuvre d'un processus d'innovation efficace peut être améliorée par l'établissement de nouvelles formes d'interactions, avec des partenaires internes et externes, basées sur l'ouverture et la collaboration. À cet égard, le développement d'approches d'IO devrait permettre de/d' :

1) Améliorer la vitesse du cycle d'innovation,

2) Diversifier les sources d'innovation, d'expertise et de connaissances, et

3) Capturer l'intelligence collective. ${ }^{87}$

Bien que l'IO présente de nombreux avantages, cette approche soulève également plusieurs questions : Comment gérer de multiples relations avec les différents partenaires, en particulier lorsque ces derniers évoluent dans divers domaines comme, par exemple, des chercheurs universitaires, des PME, des ONBL ou encore des citoyens? Qui impliquer dans une initiative d'IO? À quel moment? Comment développer et maintenir l'engagement des partenaires impliqués ? Comment

\footnotetext{
85 (Hansen and Birkinshaw 2007; Potts and Kastelle 2010)

${ }^{86}$ (Barrett et al. 2015; Lusch and Nambisan 2015; Tanaka et al. 2005)

87 (Bason 2010; Chesbrough 2006a; Chesbrough and Bogers 2014; Kankanhalli et al. 2017; West and Bogers 2017).
} 


\section{La clé des initiatives d'IO} est de bien cerner ce qu'il faut ouvrir, quand l'ouvrir, comment l'ouvrir, avec qui et comment gérer cette ouverture.

Il existe deux grandes manières de concevoir les écosystèmes, soit en tant que structure ou soit en tant qu'affiliation minimiser les conflits ? Comment s'assurer d'un partage clair et juste des propriétés intellectuelles ? Etc.

Les 10 exigent que les organisations et leurs gestionnaires déploient un ensemble d'actions afin de promouvoir I'innovation. Or, le recours à des stratégies d'IO est complexe et nécessite non seulement des ajustements des processus internes, mais également la mise en place de structures et de systèmes organisationnels spécifiques afin de supporter la participation de contributeurs externes tout au long du processus d'innovation. Ainsi, des ressources adéquates doivent être déployées afin de collecter des connaissances requises à chaque phase du processus d'innovation et être capable d'identifier des problèmes, fournir une rétroaction circulaire, concevoir et développer des solutions et des plans de mise en œuvre appropriés ${ }^{88}$. Selon différentes études, la clé du succès des initiatives $d^{\prime} 10$ est de bien cerner :

1) Ce qu'il faut ouvrir,

2) Quand l'ouvrir,

3) Comment l'ouvrir,

4) Avec qui, et

5) Comment gérer cette ouverture ${ }^{89}$.

\section{Écosystème : Comme une affiliation ou comme une structure}

Il existe deux grandes manières de concevoir les écosystèmes, soit en tant que structure ou soit en tant qu'affiliation ${ }^{90}$.

Selon la perspective "d'écosystème en tant qu'affiliation ", un écosystème représente une communauté d'organisations associées dans un contexte particulier. Les liens et les relations entre ces organisations sont définis par les réseaux auxquels ils appartiennent et participent, ainsi que par leurs affiliations à

\footnotetext{
88 (Martins et al. 2015)

89 (Huizingh 2011; King and Lakhani 2013).

90 (Adner 2017; Ganco et al. 2020; Hou and Shi 2021; Shipilov and Gawer 2020)
} 
Un écosystème se définit comme "la structure

d'alignement de

l'ensemble multilatéral de partenaires qui doivent interagir pour qu'une proposition de valeur focale se concrétise"

\section{La finalité et la fondation} d'un tel écosystème sont la matérialisation de la proposition de valeur, c.-àd. le bénéfice anticipé, par chaque organisation, en fonction de l'effort fourni des plateformes ${ }^{91}$. Comme le souligne Adner (2017), cette perspective met l'emphase sur l'effondrement des frontières entre les différents secteurs d'affaires, sur l'augmentation marquée du niveau d'interdépendances entre les organisations, aussi bien publiques que privées, dans plusieurs secteurs, ainsi que sur le potentiel des synergies entre des organisations possédant des ressources, des marchés et/ou des technologies complémentaires. La notion de réseau est au cœur de cette perspective et explique pourquoi les questions d'accès et d'ouverture, du nombre de partenaires, de la densité du réseau ainsi que de la centralité des organisations sont des préoccupations importantes aussi bien des chercheurs que des praticiens. Cette perspective focalise principalement sur les organisations, les acteurs d'écosystèmes.

Selon la perspective " d'écosystème en tant que structure ", le développement d'un écosystème s'articule autour de la structure des activités interdépendantes qui sous-tendent une proposition de valeur. Cette approche conçoit les écosystèmes comme des configurations d'activités. D'ailleurs, Adner (2017), qui adopte cette perspective, définit un écosystème comme étant « la structure d'alignement de l'ensemble multilatéral de partenaires qui doivent interagir pour qu'une proposition de valeur focale se concrétise (p.40) ${ }^{92}$. Selon cette définition, dans un écosystème, toutes les organisations ou acteurs ont des rôles/positions distincts et des flux d'activités s'opèrent entre ces organisations. La notion d'alignement ou de $\mathrm{fit}^{93} \mathrm{de}$ la structure d'un écosystème représente le degré d'accords mutuels entre les organisations par rapport à ces rôles/positions et ces flux d'activités. Par ailleurs, un écosystème est un ensemble multilatéral d'organisations ou d'acteurs, car il engage une multiplicité d'organisations dans des relations complexes et multiples, c.-à-d. non décomposables en une agrégation d'interactions bilatérales.

\footnotetext{
91 (Adner 2017)

92 "By starting with a clear definition of "ecosystem" - the alignment structure of the multilateral set of partners that need to interact in order for a focal value proposition to materialize " (Adner 2017, p. 40). 93 (Venkatraman 1989)
} 
Dans un écosystème ou un réseau, les organisations interagiront et collaboreront avec d'autres organisations qui possèdent des ressources, marchés et/ou

technologies complémentaires leur permettant tous d'améliorer leur propre performance et générer de la valeur
De plus, il est essentiel d'identifier clairement les organisations impliquées dans l'écosystème, et chacune devrait avoir comme objectif général de fournir un effort commun afin de créer de la valeur. La finalité et la fondation d'un tel écosystème sont donc la matérialisation de la proposition de valeur, c.-à-d. le bénéfice anticipé, par chaque organisation, en fonction de l'effort fourni. La perspective " d'écosystème en tant que structure » focalise donc sur l'interdépendance de ces activités entre les organisations $^{94}$.

Finalement, alors que la perspective "d'écosystème en tant qu'affiliation " débute généralement par l'identification des organisations et de leurs liens et se termine par l'identification de la possible proposition de valeur qui en découlera, la perspective "d'écosystème en tant que structure " débute plutôt par l'identification d'une proposition de valeur, et par la suite cherche à identifier l'ensemble des organisations devant $s^{\prime}$ aligner et interagir pour que la proposition se matérialise ${ }^{95}$.

\section{Écosystème vs Réseau vs Marché}

Les notions d'écosystèmes et de réseaux comportent certaines similitudes en ce sens que tous deux reposent sur le fait que, dans ces deux types de structures, les organisations fonctionnent comme des systèmes ouverts où différentes organisations ont des interactions multilatérales. Par ailleurs, les organisations qui évoluent aussi bien dans des écosystèmes ou des réseaux sont interdépendantes par rapport aux ressources, aux marchés ou/et aux technologies qui sont, du moins partiellement, sous le contrôle d'autres organisations. Dans un écosystème ou un réseau, les organisations interagiront et collaboreront ${ }^{96}$ avec d'autres organisations qui possèdent des ressources, marchés et/ou technologies complémentaires leur permettant tous d'améliorer leur propre performance et de générer de la valeur ${ }^{97}$.

\footnotetext{
94 (Adner 2017; Jacobides et al. 2018)

95 (Adner 2017)

96 (Davis 2016)

97 (Jacobides et al. 2018; Shipilov and Gawer 2020; Wang 2021)
} 


\section{Les écosystèmes sont ainsi}

formés de groupes

d'organisations, interdépendant les uns des

autres, mais dans un

environnement caractérisé par l'absence de contrôles

hiérarchiques
Différents types et niveaux d'interdépendances ${ }^{98}$ peuvent être créés, modifiés ou renforcés par les actions collectives menées par les organisations qui interagissent dans des écosystèmes ou des réseaux. Ces interdépendances varieront en fonction des divers degrés de complémentarités multilatérales et non génériques entre les organisations ${ }^{99}$. Ces complémentarités non génériques, c.-à-d. spécifiques et uniques, se manifestent lorsque la valeur engendrée par les activités d'une organisation dépend de la valeur engendrée par les activités des autres organisations. Par ailleurs, il est possible que ces interdépendances et des interactions disparaissent si les ressources, les marchés et/ou les technologies venaient à disparaitre ou à ne plus être pertinents pour les organisations. Les réseaux et les écosystèmes mettent en évidence différentes manières dont leurs organisations déploient et régulent leurs interdépendances ${ }^{100}$.

Les écosystèmes sont ainsi formés de groupes d'organisations, interdépendants les uns des autres, mais dans un environnement caractérisé par l'absence de contrôles hiérarchiques, c.-à-d. les organisations sont indépendantes les unes par rapport aux autres et ne se " possèdent " pas les unes les autres ${ }^{101}$. Pour leur part, les organisations qui évoluent dans un réseau mettent en commun leurs ressources pour atteindre un objectif commun en utilisant des mécanismes de relations interorganisationnelles structurées et formalisées par des contrats, le tout complété par des mécanismes de collaboration informels. Finalement, les écosystèmes et les réseaux diffèrent des marchés, dans la mesure où ces derniers reposent uniquement sur les prix pour coordonner les activités entre les organisations. Le tableau A1, développé par Shipilov et Gawer (2020), synthétise ces distinctions et présente un exemple pour chacun.

\footnotetext{
98 (Thompson 1967)

${ }^{99}$ (Jacobides et al. 2018)

100 (Shipilov and Gawer 2020)

101 (Jacobides et al. 2018)
} 


\begin{tabular}{|l|c|c|c|}
\hline \multicolumn{4}{|c|}{ Tableau A1. Différence entre Écosystème, Réseau et Marché (Shipilov and Gawer 2020) } \\
\hline $\begin{array}{l}\text { Mécanisme } \\
\text { de } \\
\text { coordination }\end{array}$ & $\begin{array}{c}\text { Écosystème } \\
\text { en l'absence de contrôle } \\
\text { hiérarchique complet }\end{array}$ & $\begin{array}{c}\text { Réseau } \\
\text { collaboration informelle }+ \\
\text { Complémentarités non } \\
\text { génériques }\end{array}$ & Marché \\
\hline Exemple & $\begin{array}{c}\text { L'organisation construit du } \\
\text { matériel propriétaire ; des } \\
\text { développeurs indépendants } \\
\text { écrivent les logiciels optimisés pour } \\
\text { ce matériel. Le client combine les } \\
\text { deux pour obtenir un produit final }\end{array}$ & $\begin{array}{c}\text { Des organisations de matériel } \\
\text { et de logiciels forment une } \\
\text { alliance formelle pour } \\
\text { s'engager dans un projet de } \\
\text { R\&D commun }\end{array}$ & $\begin{array}{c}\text { L'organisation } \\
\text { achète du papier à } \\
\text { un fournisseur de } \\
\text { papeterie sur la } \\
\text { base d'un prix de } \\
\text { marché }\end{array}$ \\
\hline
\end{tabular}

Les rôles clés lors de la genèse ont été regroupés

en quatre thématiques : 1)

Leadership, 2) Création

directe de valeur, 3)

Soutien à la création de

valeur et 4)

Entrepreneuriat

\section{Genèse d'écosystèmes et rôles clés}

Dans la littérature en innovation, un des facteurs clés de succès des écosystèmes est lié aux rôles clés des acteurs d'écosystèmes d'innovation ${ }^{102}$. Récemment, dans une importante revue de la littérature sur les écosystèmes d'innovation, Dedehayir et al. (2018) ont tenté d'identifier les rôles clés lors de la genèse et les ont regroupés en quatre thématiques : 1) Leadership, 2) Création directe de valeur, 3) Soutien à la création de valeur et 4) Entrepreneuriat. Un résumé de ces rôles est proposé dans les paragraphes qui suivent. Plusieurs de ces rôles font écho, de manière directe et indirecte, aux bonnes pratiques identifiées et synthétisées dans le tableau 2 et décrites dans la section précédente.

Rôles de leadership - Au niveau des rôles liés au leadership lors de la genèse des écosystèmes, un des premiers rôles identifiés est lié à la gouvernance. II est important qu'un acteur ou une organisation, comme une OSP par exemple, initie, développe et maintienne le fonctionnement d'un écosystème en clarifiant les rôles/positions des organisations évoluant dans l'écosystème et en coordonnant les interactions internes/externes en plus d'orchestrer les flux d'information et de ressources entre l'ensemble des acteurs. Un second rôle est lié à l'établissement

102 (Cusumano and Gawer 2002; Dedehayir et al. 2018; Moore 1993) 
de partenariats permettant la création d'un réseau d'organisations. II s'agit d'attirer et d'assembler des organisations possédant des ressources, marchés et/ou technologies complémentaires, de former des alliances entre des organisations de différents secteurs, de faciliter la collaboration entre ces organisations tout en encourageant les investissements complémentaires et en offrant la possibilité de créer des niches. Un troisième rôle est celui de gestionnaire de plateforme. II s'agit de concevoir, de développer et de fournir une plateforme technologique et de l'ouvrir en donnant accès aux données et à l'infrastructure afin de créer une communauté d'utilisateurs. De plus, le gestionnaire de plateforme doit assurer le maintien de l'alignement entre les organisations et leurs utilisations de la plateforme. Finalement, un dernier rôle est celui de gestionnaire de la valeur. II s'agit de favoriser la "mise en marché » de la valeur créée par les organisations de l'écosystème et d'encourager l'appropriation de la valeur pour toutes les organisations et les utilisateurs finaux.

Rôles de création de valeur - Au niveau des rôles liés à la création de valeur, il y a tout d'abord celui de fournisseur. Dans ce rôle, une ou des organisations doivent fournir et partager des ressources clés, telles que des matériaux, des expertises, des technologies, et des services, qui seront utilisés par d'autres organisations dans l'écosystème. Un autre rôle lié à la création de valeur est celui d'assembleur qui se manifeste par l'assemblage de composantes, matériaux et/ou services ainsi que par le traitement d'informations partagées par les autres organisations de l'écosystème. Un troisième rôle est celui de " complémentateur » qui doit s'assurer que les ressources et informations partagées sont compatibles avec la plateforme et répondent aux demandes/spécifications des utilisateurs finaux. Finalement, il y a le rôle d'utilisateur qui contribue à la création de valeur en définissant un problème ou un besoin, en développant des idées basées sur les données fournies et partagées dans l'écosystème tout en s'engageant dans la 
I'acquisition et l'utilisation des innovations, c.-à-d. produits ou services, créés par les organisations de l'écosystème.

Rôles de soutien à la création de valeur - Au niveau des rôles liés au soutien à la création de valeur, il y a celui de l'expert en soutien qui doit supporter les " créateurs " de valeur primaire en : 1) générant des connaissances à partir de la recherche fondamentale et appliquée, 2) fournissant du support, de l'expertise et des conseils, et 3) encourageant le transfert et la commercialisation des innovations. L'autre rôle clé est celui de champion qui doit soutenir le développement et la mise en place de l'écosystème en : établissant des connexions et des alliances entre les organisations, favorisant les interactions entre les organisations/acteurs et en facilitant l'accès aux marchés.

Rôles d'entrepreneuriat - Finalement, au niveau des rôles liés à l'entrepreneuriat, il y tout d'abord celui d'entrepreneur qui se focalise tout d'abord sur l'établissement d'une vision commune de l'écosystème. Ce rôle se manifeste par la création d'un réseau d'organisations complémentaires ainsi que par la coordination de la collaboration entre les volets de R\&D et de commercialisation. Un second rôle est celui de sponsor de l'écosystème dont l'objectif est de favoriser et soutenir la création de nouvelles initiatives. Ce rôle se manifeste en fournissant des ressources aux organisations de l'écosystème, en finançant des marchés, en achetant/co-développant des organisations et en facilitant la mise en relation entre des entrepreneurs. Finalement, il y le rôle de régulateur permettant de soutenir l'esprit entrepreneurial et de stimuler l'ouverture d'écosystèmes à de nouvelles organisations par des assouplissements réglementaires ainsi que par l'entremise de réformes économiques et politiques. 


\section{Annexe 2-Recensement défis et bonnes pratiques}

Ce projet de recherche se concentre sur l'identification de défis rencontrés par les OSP lors d'initiatives d'IO ainsi que sur l'identification de bonnes pratiques/actions organisationnelles déployées pour soutenir les initiatives d'IO ainsi que sur les rôles joués par les TI dans de telles initiatives. Pour ce faire, des études de cas " exemplaires " ${ }^{103} d^{\prime} I O$ déployées dans des OSP en contexte d'écosystème d'innovation, et ayant été des succès, ont été examinées.

Au total, ce sont 253 initiatives d'IO, lancées entre 2009 et 2020, qui ont été identifiées et sélectionnées à partir des cas documentés par l'Observatoire des Innovations du Secteur public (OPSI) de l'OCDE (https://oecd-opsi.org/our-work/casestudies/). Afin d'enrichir notre compréhension de ces cas, nous avons également collecté différents documents sur chacun des cas, incluant des articles de presse (recherchés à partir de la base de données Factiva) et des publications académiques ou pour praticiens (recherchées à partir des bases de données ABI/Inform Global et Business Source Premier).

Parmi les études de cas analysées, plus de la moitié (139/253) portent sur des innovations de produits alors que le quart (69/253) des études de cas focalisent sur des innovations organisationnelles. Bien entendu, toutes les études de cas sélectionnées décrivent des initiatives d'IO impliquant des OSP, c'est-à-dire qu'elles devaient, au niveau des partenaires impliqués, inclure minimalement une OSP ( $n=253)$ ainsi qu'au moins un autre type de partenaires. Parmi les autres types de partenaires impliqués, ce sont les organisations privées ( $n=169$ ) et les citoyens $(n=103)$ que l'on retrouve le plus souvent dans les initiatives d'IO analysées. Le tableau A2 présente les données descriptives de ces études de cas.

103 Source : https://oecd-opsi.org/our-work/case-studies/ 


\begin{tabular}{|c|l|c|}
\hline Dimensions & \multicolumn{1}{|c|}{ Détails } & Nb. de cas \\
\hline \multirow{4}{*}{$\begin{array}{c}\text { Types } \\
\text { d'innovations }\end{array}$} & Innovation de communication & 18 \\
\cline { 2 - 3 } & Innovation organisationnelle & 69 \\
\cline { 2 - 3 } & Innovation de processus & 27 \\
\cline { 2 - 3 } & Innovation de produit & 139 \\
\hline \multirow{4}{*}{$\begin{array}{c}\text { Partenaires } \\
\text { impliqués }\end{array}$} & OSP & 253 \\
\cline { 2 - 3 } & Organisations privées & 169 \\
\cline { 2 - 3 } & Citoyens & 103 \\
\cline { 2 - 3 } & Chercheurs & 16 \\
\hline
\end{tabular}

Tableau A2 : Description des projets 10

L'OPSI est un organisme sans but lucratif (OSBL) qui recueille et catégorise des projets d'IO exemplaires pour construire une base de données étendue d'études de cas provenant de plus de 60 pays. L'objectif de l'OPSI est d'encourager l'innovation dans le secteur public en facilitant le partage des connaissances. Dans chaque cas, un accent particulier a été mis sur le partage des défis rencontrés, les décisions clés et les actions déployées. Afin d'être incluse dans notre échantillon, une étude de cas doit avoir déployé une approche d'IO, c'est-à-dire avoir impliqué au moins une OSP et un autre partenaire (par exemple citoyen, groupes communautaires, organisation privée, autre gouvernement agences, etc.).

Dans un premier temps, les défis rencontrés et les bonnes pratiques/actions organisationnelles ${ }^{105}$ utilisées pour relever les différents défis lors des projets IO ont été codés à l'aide du logiciel NVivo pour chacune des 253 études de cas. Ensuite, les défis et les pratiques/actions organisationnelles similaires ont été regroupés et des étiquettes ont été créées pour chacune d'entre elles. Afin d'accroître la fiabilité des résultats, un des auteurs et un assistant de recherche ont codifié indépendamment 100 études de cas et ont comparé leurs résultats jusqu'à ce qu'un consensus soit atteint. Finalement, les pratiques/actions organisationnelles codifiées ont été 
catégorisées, en fonction de leurs objectifs sous-jacents, à l'une des trois principales capacités dynamiques soit: Détection, Capture ou Transformation ${ }^{106}$.

Bien que les 253 études de cas analysées afin d'identifier les défis et les bonnes pratiques/actions organisationnelles, certaines réserves doivent être prises en considération lors de I'interprétation de ces résultats. Tout d'abord, même si les études de cas analysées sont des cas "exemplaires" de déploiements d'initiatives d'IO impliquant des OSP, les données utilisées n'ont pas été directement collectées par les auteurs ce qui peut limiter la richesse et la contextualisation de l'interprétation. De plus, les cas ne sont pas exhaustifs, car la longueur moyenne de ceux-ci est d'environ six pages à simple interligne. Toutefois, l'utilisation de données secondaires est une pratique fréquemment utilisée en recherche et très efficace.

De plus, la longueur limitée des cas est contrebalancée par le très grand nombre de cas analysés $(\mathrm{N}=253)$. Par ailleurs, la structure et le contenu des études de cas focalisaient toutes sur les éléments essentiels pour notre étude puisque chaque cas présente: 1) une brève description de I'innovation, c.-à-d. caractéristique innovante de l'initiative et l'état actuel de l'IO; 2) le processus de développement de l'innovation c.-à-d. collaborations et partenariats ainsi qu'usagés, partenaires impliqués et bénéficiaires, et 3) réflexion sur I'IO, c.-à-d. résultats et impacts, défis et écueils, conditions de succès, leçons apprises.

Il faut également noter que les cas analysés sont des cas " exemplaires », c'est-à-dire que ce sont des cas jugés comme étant des succès. Il est important de souligner la possibilité d'un biais de sélection. Par contre, compte tenu de l'objectif de notre étude, qui est d'identifier les défis et les bonnes pratiques/actions organisationnelles associées aux initiatives de $1 O$ impliquant des OSP, un tel biais n'est pas problématique,

106 (Teece, 2017) 
bien au contraire. Finalement, l'un des éléments absents des études de cas découle du fait que, dans plusieurs cas, il n'est pas possible d'identifier exactement (il faut tenter de le déduire) qui a initié, déployé et/ou assuré la réalisation des bonnes pratiques/actions organisationnelles. 


\section{Annexe 3 - Études de cas : PULSAR et CISAM}

Avant même de documenter les deux études de cas présentées dans le présent rapport, la première étape a été d'établir les critères d'inclusion et d'exclusion des études de cas. Le premier critère d'inclusion était qu'il s'agisse bien d'un écosystème et non d'un réseau, d'une alliance ou de toutes autres formes de structures $d$ 'interactions formelles entre organisations. Pour être sélectionnées, les études de cas devaient donc impliquer un ensemble multilatéral d'organisations, interagissant les unes avec les autres dans le but de développer une proposition de valeur partagée. De plus, ces organisations devaient posséder des ressources, marchés et/ou technologies complémentaires. Le second critère d'inclusion était qu'une ou plusieurs OSP jouent un rôle central dans la mise en place, le développement et/ou le maintien de ces écosystèmes. Finalement, le dernier critère d'inclusion était " opérationnel » en ce sens que, pour être retenu, les chercheurs devaient : 1) pouvoir accéder à certains documents clés décrivant la genèse et le fonctionnement de l'écosystème, 2) effectuer des entrevues avec des acteurs clés, et 3) pouvoir nommer le nom des écosystèmes et des organisations impliquées.

Compte tenu que le nombre d'écosystèmes d'innovations existant sur la planète est relativement limité et que l'accès à ces terrains d'études est parfois difficile à obtenir, le processus d'identification, de sélection et de négociation avec les écosystèmes fut laborieux et plus long qu'anticipé. Par ailleurs, il faut noter que la collecte de données qui devait initialement débuter en mars 2020 a été repoussée d'une année compte tenu des mesures sanitaires et des changements organisationnels engendrés par la pandémie de COVID-19. Finalement, grâce à la générosité et l'ouverture des responsables, aussi bien chez PULSAR qu'à la CISAM, les premières entrevues ont pu débuter en mars 2021 et se sont échelonnées jusqu'en novembre 2021. Un guide d'entrevue a été développé est disponible à l'annexe 4. 
Au total nous avons effectué dix entrevues, soit cinq chez PULSAR et cinq à la CISAM. Les entrevues ont duré en moyenne 75 minutes. Lors de chaque entrevue, au moins deux chercheurs étaient présents. La majorité des entrevues ont été enregistrées et des notes ont été prises lors des entrevues. Ces notes et observations ont par la suite été partagées avec les autres chercheurs. Chez PULSAR, nous avons rencontré le directeur scientifique, la coordonnatrice scientifique, la gestionnaire responsable de la genèse de l'écosystème, une responsable des produits ainsi qu'un analyste en évaluation de la santé durable. Chez CISAM, nous avons rencontré le directeur principal de la CISAM, une business developer responsable des mises en relation, un business developer responsable du volet numérique, un business developer responsable du volet science humaine et sociale et un business developer responsable du volet santé. En plus des données provenant des entrevues, nous avons eu accès à plusieurs documents décrivant aussi bien le fonctionnement, la vision ainsi que la genèse de ces deux écosystèmes. Les descriptions des deux études de cas présentées dans le présent rapport se basent sur les données provenant des entrevues et des documents. Par ailleurs, les informations décrivant PULSAR et CISAM et présentées ici ont été validées par des représentants de chacun des deux écosystèmes. 


\section{Annexe 4-Guide d'entrevue}

\section{Section 0 - Mise en contexte}

L'entrevue devrait durer environ 60-70 minutes. Au cours de l'entrevue, il vous sera demandé de partager votre perception par rapport au phénomène de l'innovation ouverte et des écosystèmes d'innovation ( $\mathrm{Tl})$, et surtout de nous décrire le projet d'innovation ouverte/écosystème d'innovation lié au (insérer nom organisation ici)

Est-ce que vous permettrez que j'enregistre l'entrevue?

Si la réponse est non, alors uniquement des notes manuscrites seront prises.

\section{Section 1.1 - Information sur le/la répondant/te}

1. Quel est votre titre?

2. Depuis combien de temps travaillez-vous dans ce poste?

3. Quelle est votre expérience dans le domaine de l'innovation ?

a. Nombre d'années d'expérience ? Nombre de projets?

4. Quel est votre « background » (c.-à-d. parcours) ?

5. Avez-vous eu une formation liée à l'innovation ? Si, oui, de quoi s'agit-il?

\section{Section 1.2 - Conceptualisation de l'innovation ouverte}

1. Pour vous, qu'est-ce l'expression « innovation ouverte » signifie en général ? Qu'est-ce que c'est?

a. Plus particulièrement dans le contexte de votre organisation?

2. Comment définiriez-vous la notion d'innovation ouverte?

a. En termes d'actions ? En termes de structure ? En termes de processus ? En termes de connaissances?

b. Quels sont les éléments les plus importants de l'innovation ouverte ? Pourquoi ?

c. Est-ce que ces actions / structure / processus / connaissances ont toujours la même importance pour toutes les initiatives d'innovation ouverte?

\section{Section 1.3 - Conceptualisation d'écosystème d'innovation}

1. Pour vous, qu'est-ce l'expression « écosystème d'innovation » signifie en général ? Qu'est-ce que c'est?

a. Plus particulièrement dans le contexte de votre organisation?

2. Comment définiriez-vous la notion d'écosystème d'innovation ?

a. En termes de structure ? En termes de participantes ? En termes de rôles ?

b. Quels sont les éléments les plus importants d'un écosystème d'innovation ?

Pourquoi ?

c. Est-ce que ces structures/ participants / rôles sont toujours de la même importance pour toutes les initiatives d'innovation ouverte?

Section 1.4 - Innovation ouverte, défis, barrières et "drivers " 
1. Selon vous, quels sont les avantages pour une organisation comme (insérer le nom de l'organisation), de se lancer, de s'impliquer, dans une initiative d'innovation ouverte ?

2. Sur la base de quel(s) facteur(s), est-ce qu'une organisation se base pour se lancer dans une initiative d'innovation ouverte?

3. Lorsque vous pensez aux initiatives d'innovation ouverte auxquelles vous avez participé (ou pas), quels sont, selon vous, les principaux défis liés :

a. À la mise en place d'initiatives d'innovation ouverte ?

b. Au bon fonctionnement d'initiatives d'innovation ouverte ?

c. À la réussite d'initiatives d'innovation ouverte?

4. Pour chacun des 5-6 défis les plus importants identifiés, quelle(s) stratégie(s) a(ont) été utilisée(s) ou peut-être utilisée(s) pour surmonter chacun des défis ?

5. Lors d'initiatives d'innovation ouverte, quelles sont, selon vous, les principales barrières à la réussite de ce genre d'initiative?

a. Par exemple : Manque de flexibilité dans les lois ; Absence d'incitatifs ; Manque de financement ; Risque d'échec ; Niveau de confiance des parties prenantes; Manque de coopération dans l'organisation; Obstacles internes (temps ou incitation, coordination, logistique, épuisement professionnel, résistance bureaucratique, technologie, syndicats, cadres intermédiaires, opposants à l'entrepreneur public; Obstacles externes (règles, fournisseurs, utilisateurs résistants, réglementation législative, opposition politique, transition politique, doute public, atteindre le groupe cible, intérêts affectés, concurrents)

6. Selon vous, lors d'initiatives d'innovation ouverte, quelles tactiques/stratégies peuvent être déployées par les organisations afin de surmonter les principales barrières à la réussite ?

a. Par exemple : Tactiques de persuasion (avantages sociaux, marketing social, projet de démonstration); Tactiques d'adaptation (consultation, coopération, formation offerte, programme adapté à la culture, compensation); Autres tactiques (recherche de ressources, persévérance, résolution de problèmes logistiques, recherche de soutien en bureaucratie, renforcement du soutien politique, concentration, vision claire, modification de la technologie, changement des lois, reconnaissance, gestionnaires du changement)

7. Comment est-ce possible d'évaluer l'impact d'initiatives d'innovation ouverte / écosystème d'innovation?

8. Comment est-ce possible d'évaluer le succès d'initiatives d'innovation ouverte / écosystème d'innovation?

\section{Section 1.5 - CAS - Innovation ouverte - Description}

***Note : Faire des liens avec questions précédentes : facteurs, avantages, barrières, structures, parties prenantes, etc.

1. Pourriez-vous me décrire l'initiative d'innovation ouverte/écosystème entourant la mise sur pied et le fonctionnement de (insérer le nom de l'organisation) ?

a. Qu'est-ce que le (insérer le nom de l'organisation) ?

i. Quand et dans quel contexte est-ce que cette initiative a-t-elle été lancée ?

b. Pourquoi cette initiative a-t-elle vu le jour ? - Facteurs déterminants

i. Quel était le ou les objectifs principaux visés?

c. Comment cette initiative d'innovation ouverte /écosystème d'innovation a-t-elle été initiée ?

i. Par qui ?

d. Quelles ont été les grandes étapes de mise sur pied ?

e. Comment une initiative d'innovation ouverte /écosystème fonctionne-t-elle ?

i. Quelle est sa structure ? Ses principaux processus ? 
f. Qui sont les principaux collaborateurs (c.-à-d. les principales parties prenantes) ?

i. Ont-ils tous les mêmes rôles ? Quels sont chacun des rôles ?

g. Comment cette initiative a-t-elle évoluée à travers le temps ?

i. Quels ont été les principaux changements/transformations ?

h. Quelles ont été les principales barrières ? Les défis ?

i. Quels ont été les extrants ? Les résultats?

2. Qui a collaboré à cette initiative d'innovation ouverte /écosystème d'innovation ?

a. Par exemple : Employés ? Gouvernement - Organismes publics ? Partenaires externes ? Partenaires internes ? Citoyens / Utilisateurs ? Organisations privées ? Communauté ? Universités, centres de recherche ?

3. Pour chaque collaborateur, quel a été :

a. Son/ses rôle(s) ?

b. Son type de participation : Informer, consulter, impliquer, collaborer, responsabiliser?

c. Sa contribution : connaissances, \$, rétroactions, idées, besoins, demandes, idées, solutions, problèmes, conceptions, etc. ?

d. Ses moments d'implication : formulation du problème, génération d'idées, sélection d'idées, conversion d'idées, diffusion d'idées ?

e. Ses incitatifs à participer au projet ?

f. Ses pouvoirs?

4. Quels ont été les éléments clés/facteurs clés de succès de l'initiative d'innovation ouverte /écosystème d'innovation?

\section{Section 1.6 - CAS - Innovation ouverte - Stratégies}

1. *Quelles stratégies et pratiques ont été mises ne place pour assurer le bon fonctionnement de l'innovation ouverte /écosystème d'innovation?

a. Quelles stratégies et pratiques ont BIEN fonctionné ? Pourquoi ?

b. Quelles stratégies et pratiques ont MOINS BIEN fonctionné ? Pourquoi ?

2. *Comment l'application de ces stratégies et pratique a permis d'atténuer ou non les problématiques rencontrées?

3. Quel(s) critères sont utilisés pour évaluer le succès / réussite de l'innovation ouverte /écosystème d'innovation?

a. Ont-ils changé à travers le temps ? Si oui, pourquoi ?

\section{Section 1.7 - Validation des bonnes pratiques/actions}

1. Lors d'une revue de littérature, nous avons identifié, sur la base de plusieurs dizaines de cas d'innovation ouvertes déployées par des organisations du secteur public, 18 bonnes pratiques/actions organisationnelles (voir tableau ci-dessous).

2. Pour chacune de ces pratiques/actions organisationnelles, pourriez-vous nous dire :

a. Premièrement, ce que vous en pensez ? Votre impression ?

b. Deuxièmement, est-ce que cette pratique/action organisationnelle a été déployée dans votre initiative?

i. Si oui, comment ? Qu'est-ce que cela a donné ?

ii. Sinon, est-ce que ça aurait été possible et utile ? Pourquoi ? 


\section{Références bibliographiques}

Adner, R. 2006. "Match Your Innovation Strategy to Your Innovation Ecosystem," Harvard business review (84:4), p. 98.

Adner, R. 2017. "Ecosystem as Structure: An Actionable Construct for Strategy," Journal of management (43:1), pp. 39-58.

Adner, R., and Euchner, J. 2014. "Innovation Ecosystems," Research-Technology Management (57:6), pp. 10-14.

Adner, R., and Kapoor, R. 2010. "Value Creation in Innovation Ecosystems: How the Structure of Technological Interdependence Affects Firm Performance in New Technology Generations," Strategic management journal (31:3), pp. 306-333.

Barrett, M., Davidson, E., Prabhu, J., and Vargo, S. L. 2015. "Service Innovation in the Digital Age: Key Contributions and Future Directions," MIS quarterly (39:1), pp. 135154.

Bason, C. 2010. Leading Public Sector Innovation: Co-Creating for a Better Society. Great Britain: Policy Press.

Bekkers, V., Edelenbos, J., and Steijn, B. 2011. Innovation in the Public Sector: Linking Capacity and Leadership. New York, NY: Palgrave Macmillan.

Bennett, N., and Lemoine, J. 2014. "What Vuca Really Means for You," Harvard business review (92:1/2).

Bommert, B. 2010. "Collaborative Innovation in the Public Sector. ," International Public Management Review (11:1), pp. 15-53.

Borins, S. 2001. "Encouraging Innovation in the Public Sector," Journal of intellectual capital (2:3), pp. 310-319.

Borins, S. 2014. The Persistence of Innovation in Government a Guide for Innovative Public Servants. www.businessofgovernment.org: IBM Center for the Business of Government.

Carter, L., and Bélanger, F. 2005. "The Utilization of EGovernment Services: Citizen Trust, Innovation and Acceptance Factors," Information systems journal (15:1), pp. 5-25.

Cheng, C. C., and Huizingh, E. K. 2015. "Identifying Open Innovation Capabilities: A Critical Literature Review," ISPIM Conference Proceedings: The International 
Society for Professional Innovation Management (ISPIM), p. 1.

Chesbrough, H. W. 2006a. "Open Innovation: A New Paradigm for Understanding Industrial Innovation," in Openinnovation: Researching a New Paradigm, $\mathrm{H}$. Chesbrough, W. Vanhaverbeke and J. West (eds.). Oxford: Oxford University Press, pp. 1-12.

Chesbrough, H. W. 2006b. Open Innovation: The New Imperative for Creating and Profiting from Technology. Harvard Business Press.

Chesbrough, H. W., and Bogers, M. 2014. "Explicating Open Innovation: Clarifying an Emerging Paradigm for Understanding Innovation," in Open Innovation: New Frontiers and Applications, $\mathrm{H}$. Chesbrough, W. Vanhaverbeke and J. West (eds.). Oxford: Oxford University Press, pp. 3-28.

Cusumano, M. A., and Gawer, A. 2002. "The Elements of Platform Leadership," MIT Sloan management review (43:3), p. 51.

Damanpour, F., and Schneider, M. 2009. "Characteristics of Innovation and Innovation Adoption in Public Organizations: Assessing the Role of Managers," Journal of public administration research and theory (19:3), pp. 495-522.

Davis, J. P. 2016. "The Group Dynamics of Interorganizational Relationships: Collaborating with Multiple Partners in Innovation Ecosystems," Administrative science quarterly (61:4), pp. 621-661.

De Vries, H., Bekkers, V., and Tummers, L. 2015. "Innovation in the Public Sector: A Systematic Review and Future Research Agenda," Public Administration).

De Vries, H., Bekkers, V., and Tummers, L. 2016. "Innovation in the Public Sector: A Systematic Review and Future Research Agenda," Public administration (94:1), pp. 146-166.

Dedehayir, O., Mäkinen, S. J., and Ortt, J. R. 2018. "Roles During Innovation Ecosystem Genesis: A Literature Review," Technological Forecasting and Social Change (136), pp. 18-29.

Duval, M., and Speidel, K. 2014. Open Innovation. Développez Une Culture Ouverte Et Collaborative Pour Mieux Innover. Paris, France: Dunod. 
Edmondson, A. C. 2016. "Wicked Problem Solvers," Harvard business review (94:6), pp. 52-59, 117.

Eggers, W. D., and Singh, S. K. 2009. The Public Innovator's Playbook: Nurturing Bold Ideas in Government. Ash Institute, Harvard Kennedy School.

Fernandez, S., and Rainey, H. G. 2006. "Managing Successful Organizational Change in the Public Sector," Public administration review (66:2), pp. 168-176.

Ganco, M., Kapoor, R., and Lee, G. K. 2020. "From Rugged Landscapes to Rugged Ecosystems: Structure of Interdependencies and Firms' Innovative Search," Academy of Management Review (45:3), pp. 646-674.

Granstrand, O., and Holgersson, M. 2020. "Innovation Ecosystems: A Conceptual Review and a New Definition," Technovation (90), p. 102098.

Green, R., Roos, G., Agarwal, R., and Scott-Kemmis, D. 2014. "Shaping the Future through Co-Creation," Institute of Public Administration Australia, Retrieved in June 2017 from

http://www.ipaa.org.au/documents/2014/05/innovati on-report.pdf.

Hansen, M. T., and Birkinshaw, J. 2007. "The Innovation Value Chain," Harvard business review (85:6), p. 121.

Hartley, J., Sørensen, E., and Torfing, J. 2013. "Collaborative Innovation: A Viable Alternative to Market Competition and Organizational Entrepreneurship," Public Administration Review (73:6), pp. 821-830.

Helfat, C. E., Finkelstein, S., Mitchell, W., Peteraf, M. A., Singh, H., Teece, D. J., and Winter, S. G. 2007. Dynamic Capabilities: Understanding Strategic Change in Organizations. Malden: Blackwell Publishing.

Helfat, C. E., and Peteraf, M. A. 2003. "The Dynamic ResourceBased View: Capability Lifecycles," Strategic management journal (24:10), pp. 997-1010.

Hilgers, D., and Ihl, C. 2010. "Citizensourcing: Applying the Concept of Open Innovation to the Public Sector," The International Journal of Public Participation (4:1), pp. 67-88.

Hoon, C. 2013. "Meta-Synthesis of Qualitative Case Studies an Approach to Theory Buildin," Organizational Research Methods (16:4). 
Hou, H., and Shi, Y. 2021. "Ecosystem-as-Structure and Ecosystem-as-Coevolution: A Constructive Examination," Technovation (100), p. 102193.

Huizingh, E. K. 2011. "Open Innovation: State of the Art and Future Perspectives," Technovation (31:1), pp. 2-9.

Jacobides, M. G., Cennamo, C., and Gawer, A. 2018. "Towards a Theory of Ecosystems," Strategic Management Journal (39:8), pp. 2255-2276.

Kankanhalli, A., Zuiderwijk, A., and Tayi, G. K. 2017. "Open Innovation in the Public Sector: A Research Agenda," Government Information Quarterly (34:1), pp. 84-89.

Kim, S. D. 2017. "Characterization of Unknown Unknowns Using Separation Principles in Case Study on Deepwater Horizon Oil Spill," Journal of Risk Research (20:1), pp. 151-168.

King, A., and Lakhani, K. R. 2013. "Using Open Innovation to Identify the Best Ideas," MIT Sloan management review (55:1), p. 41.

Koppenjan, J. F. M., and Klijn, E.-H. 2004. Managing Uncertainties in Networks: A Network Approach to Problem Solving and Decision Making. Psychology Press.

Larsson, R. 1993. "Case Survey Methodology: Quantitative Analysis of Patterns across Case Studies," Academy of Management Journal (36:6), pp. 1515-1546.

Linders, D. 2012. "From E-Government to We-Government: Defining a Typology for Citizen Coproduction in the Age of Social Media," Government Information Quarterly (29:4), pp. 446-454.

Lusch, R. F., and Nambisan, S. 2015. "Service Innovation: A Service-Dominant Logic Perspective," MIS Quarterly (39:1), pp. 155-175.

Martins, T. C. M., de Souza Bermejo, P. H., and de Souza, W. V. B. 2015. "Open Innovation for Citizen Coproduction," International Conference on Electronic Government and the Information Systems Perspective: Springer, pp. 177188.

Meijer, A. 2019. "Public Innovation Capacity: Developing and Testing a Self-Assessment Survey Instrument," International Journal of Public Administration (42:8), pp. 617-627.

Mergel, I. 2015. "Opening Government: Designing Open Innovation Processes to Collaborate with External 
Problem Solvers," Social Science Computer Review (33:5), pp. 599-612.

Mergel, I. 2018. "Open Innovation in the Public Sector: Drivers and Barriers for the Adoption of Challenge. Gov," Public Management Review (20:5), pp. 726-745.

Moore, J. F. 1993. "Predators and Prey: A New Ecology of Competition," Harvard business review (71:3), pp. 7586.

Oh, D.-S., Phillips, F., Park, S., and Lee, E. 2016. "Innovation Ecosystems: A Critical Examination," Technovation (54), pp. 1-6.

Osterwalder, A., Viki, T., and Pigneur, P. 2019. "Why Your Organization Needs an Innovation Ecosystem," Havard Business Review (November).

Pavlou, P. A., and El Sawy, O. A. 2006. "From It Leveraging Competence to Competitive Advantage in Turbulent Environments: The Case of New Product Development," Information Systems Research (17:3), pp. 198-227.

Pavlou, P. A., and El Sawy, O. A. 2010. "The "Third Hand": ItEnabled Competitive Advantage in Turbulence through Improvisational Capabilities," Information Systems Research (21:3), pp. 443-443-471,656-657.

Piller, F. T., Vossen, A., and Ihl, C. 2012. "From Social Media to Social Product Development: The Impact of Social Media on Co-Creation of Innovation," Die Unternehmung (65:1).

Pollitt, C., and Bouckaert, G. 2011. Public Management Reform: A Comparative Analysis-New Public Management, Governance, and the Neo-Weberian State. Oxford University Press.

Potts, J., and Kastelle, T. 2010. "Public Sector Innovation Research: What's Next?," Innovation (12:2), pp. 122137.

Rogers, E. M. 2003. Diffusion of Innovations, (5th ed.). New York, NY: Free Press.

Russo-Spena, T., and Mele, C. 2012. "Five Co-S" in Innovating: A Practice-Based View," Journal of Service Management (23:4), pp. 527-553.

Sambamurthy, V., Bharadwaj, A., and Grover, V. 2003. "Shaping Agility through Digital Options: Reconceptualizing the Role of Information Technology in Contemporary Firms," MIS quarterly), pp. 237-263. 
Schilke, O., Hu, S., and Helfat, C. E. 2018. "Quo Vadis, Dynamic Capabilities? A Content-Analytic Review of the Current State of Knowledge and Recommendations for Future Research," Academy of Management Annals (12:1), pp. 390-439.

Shipilov, A., and Gawer, A. 2020. "Integrating Research on Interorganizational Networks and Ecosystems," Academy of Management Annals (14:1), pp. 92-121.

Sørensen, E., and Torfing, J. 2006. "Metagoverning Collaborative Innovation in Governance Networks," The American Review of Public Administration), $p$. 0275074016643181.

Sørensen, E., and Torfing, J. 2011. "Enhancing Collaborative Innovation in the Public Sector," Administration \& Society (43:8), pp. 842-868.

Stewart-Weeks, M., and Kastelle, T. 2015. "Innovation in the Public Sector," Australian Journal of Public Administration (74:1), pp. 63-72.

Talmar, M., Walrave, B., Podoynitsyna, K. S., Holmström, J., and Romme, A. G. L. 2020. "Mapping, Analyzing and Designing Innovation Ecosystems: The Ecosystem Pie Model," Long Range Planning (53:4), p. 101850.

Tanaka, N., Glaude, M., and Gault, F. 2005. Oslo Manual Guidelines for Collecting and Interpreting Innovation Data. Paris, France: OECD Publishing.

Tat-Kei Ho, A. 2002. "Reinventing Local Governments and the E-Government Initiative," Public administration review (62:4), pp. 434-444.

Teece, D. J., Peteraf, M., and Leih, S. 2016. "Dynamic Capabilities and Organizational Agility: Risk, Uncertainty, and Strategy in the Innovation Economy," California Management Review (58:4), pp. 13-35.

Teece, D. J., Pisano, G., and Shuen, A. 1997. "Dynamic Capabilities and Strategic Management," Strategic Management Journal (18:7), pp. 509-509.

Thompson, J. D. 1967. Organizations in Action. New York: McGraw-Hill.

Tsujimoto, M., Kajikawa, Y., Tomita, J., and Matsumoto, Y. 2018. "A Review of the Ecosystem Concept-Towards Coherent Ecosystem Design," Technological Forecasting and Social Change (136), pp. 49-58.

Venkatraman, N. 1989. "Strategic Orientation of Business Enterprises: The Construct, Dimensionality, and 
Measurement," Management Science (35:8), pp. 942962.

Vigoda-Gadot, E., Shoham, A., Schwabsky, N., and Ruvio, A. 2008. "Public Sector Innovation for Europe: A Multinational Eight-Country Exploration of Citizen's Perspectives," Public Administration (86:2), pp. 307329.

Wallin, M. W., and Von Krogh, G. 2010. "Organizing for Open Innovation:: Focus on the Integration of Knowledge," Organizational dynamics (39:2), pp. 145-154.

Wang, P. 2021. "Connecting the Parts with the Whole: Toward an Information Ecology Theory of Digital Innovation Ecosystems," MIS Quarterly (45:1).

Wernerfelt, B. 1984. "A Resource-Based View of the Firm," Strategic management journal (5:2), pp. 171-180.

West, J., and Bogers, M. 2017. "Open Innovation: Current Status and Research Opportunities," Innovation (19:1), pp. 43-50.

West, J., Salter, A., Vanhaverbeke, W., and Chesbrough, H. W. 2014. "Open Innovation: The Next Decade." Elsevier.

Wiesel, F., and Modell, S. 2014. "From New Public Management to New Public Governance? Hybridization and Implications for Public Sector Consumerism," Financial Accountability \& Management (30:2), pp. 175-205.

Yeow, A., Soh, C., and Hansen, R. 2018. "Aligning with New Digital Strategy: A Dynamic Capabilities Approach," The Journal of Strategic Information Systems (27:1), pp. 4358. 